University of Tennessee Health Science Center

UTHSC Digital Commons

$12-2012$

\title{
Effect of Compression Force on Agglomeration of Micronized Active Pharmaceutical Ingredients: Techniques to Prevent API Agglomeration During Compression
}

\author{
Suresh Potharaju \\ University of Tennessee Health Science Center
}

Follow this and additional works at: https://dc.uthsc.edu/dissertations

Part of the Pharmaceutics and Drug Design Commons

\section{Recommended Citation}

Potharaju, Suresh, "Effect of Compression Force on Agglomeration of Micronized Active Pharmaceutical Ingredients: Techniques to Prevent API Agglomeration During Compression" (2012). Theses and Dissertations (ETD). Paper 213. http://dx.doi.org/10.21007/etd.cghs.2012.0251.

This Dissertation is brought to you for free and open access by the College of Graduate Health Sciences at UTHSC Digital Commons. It has been accepted for inclusion in Theses and Dissertations (ETD) by an authorized administrator of UTHSC Digital Commons. For more information, please contact jwelch30@uthsc.edu. 


\title{
Effect of Compression Force on Agglomeration of Micronized Active Pharmaceutical Ingredients: Techniques to Prevent API Agglomeration During Compression
}

\author{
Abstract \\ Micronization is one of the common processes for size reduction to increase surface area of poorly \\ soluble Active Pharmaceutical Ingredient's (API). This size reduction improves the dissolution rate and \\ permeability thereby increasing the bioavailability for hydrophobic API's.
}

Tablets and capsules are the most marketed and easy to manufacture solid dosage forms. During manufacturing of tablets, high compression forces are applied uniaxially on the powder bed to get a coherent consolidated compact with good tensile strength. So, diluents are required to mix with API's and compress into tablets. When this mixture is compressed into tablets, there is a possibility of agglomeration of micronized API to API particles together within the tablet due to their high surface area and high surface free energy during compression. Increasing the particle size and decreasing the surface is generally expected to decrease the dissolution rate of the API.

Micronized furosemide and griseofulvin were used as model API's based on the biopharmaceutical classification system to study the effect of compression force on particle agglomeration during compression and also to study the processes and materials to prevent that agglomeration. The size of furosemide and griseofulvin particles was measured after disintegration of tablets compressed by varying compression forces and drug loading. Effect of size of diluents on agglomeration of API's was also studied by varying the size of diluents like lactose mono hydrate and dicalcium phosphate dihydrate. The bonding mechanism for formation of agglomerates of these API's during compression was studied by comparing the tensile strength of the compacts soaked and dried in solvents of various dielectric media. Prevention of micronized particle agglomeration during compression was studied by using particle agglomeration inhibitors like PEG 3350, Lutrol F68 micro, hydroxyl propyl starch polymer and by various treatment methods like physical mixing, hexane slurry method, roller compaction and spray drying. The particles were characterized for change in crystal structure, polymorphism and surface morphology by X-ray diffraction, thermal studies and microscopy studies.

Micronized furosemide and griseofulvin particle size was increased significantly and linearly by increasing the drug loading and compression force during compression. The size of the formed agglomerates was directly proportional to particle size of diluents. The dominating bonding mechanism responsible for agglomerate formation was found to be solid bridge formation between drug particles during compression. The API to API particle agglomeration during compression was successfully prevented by using particle agglomeration inhibitors (PAl's). Treatment with hexane and increase in the level of super disintegrant provided limited prevention of agglomeration during compression for furosemide particles. The spray drying with either mannitol or hydroxy propyl starch for micronized furosemide significantly reduced the agglomeration of API particles during compression. Roller compaction process with Lutrol F68 micro and PEG 3350 provided significant reduction in the agglomeration of drug particles for both the API's during compression. Compression force and treatment with PAl's on polymorphism was observed in changing the crystal structure and polymorphism during compression and confirmed by thermal and X-ray crystallographic studies for both the API's. Scanning Electron Microscopy studies revealed compression force and roller compaction process with PAl's changed the surface morphology of both the API's.

The results of the above studies indicated that compression of micronized hydrophobic poorly soluble drugs into tablets affects the dissolution rate due to agglomeration of API. The micronization process for improving the dissolution rate of poorly soluble drugs showed disadvantageous with respect to tablets 
compressed at high compression forces because of agglomeration of furosemide and griseofulvin particles during compression. Hence control of particle size and selection of size of diluents are necessary during formulation of tablets of hydrophobic poorly soluble drugs. Primary particle size increase of micronized hydrophobic drugs during compression can also be minimized by treatment with particle agglomeration inhibitors prior to compression.

\section{Document Type}

Dissertation

Degree Name

Doctor of Philosophy $(\mathrm{PhD})$

\section{Program}

Pharmaceutical Sciences

\section{Research Advisor}

James R. Johnson, Ph.D.

\section{Keywords}

Compression, Lutrol F68 micro, Micronization, Particle Agglomeration Inhibitors, Roller compaction, Spray drying

\section{Subject Categories}

Medicine and Health Sciences | Pharmaceutics and Drug Design | Pharmacy and Pharmaceutical Sciences 
Effect of Compression Force on Agglomeration of Micronized Active Pharmaceutical Ingredients: Techniques to Prevent API Agglomeration during Compression

\author{
A Dissertation \\ Presented for \\ The Graduate Studies Council \\ The University of Tennessee \\ Health Science Center
}

In Partial Fulfillment

Of the Requirements for the Degree

Doctor of Philosophy

From The University of Tennessee

By

Suresh Potharaju

December 2012 
Copyright $(\mathcal{}) 2012$ by Suresh Potharaju. All rights reserved. 


\section{DEDICATION}

I dedicate this work to my wife Samadhana Jyothi Mortha and my son Ivan Potharaju and my parents Mrs. Lillimma Potharaju and Mr. Prasada rao Potharaju (deceased) for their endless love and encouragement. 


\section{ACKNOWLEDGEMENTS}

First of all I would like to thank almighty GOD, Jesus Christ for giving me strength, wisdom and showing hope in the events of troubles and pains. Without his grace I could not achieve this milestone.

I would like to give my heartily thanks to our deceased Professor Dr. Atul J. Shukla for providing me an opportunity to pursue a doctoral degree in Unites States of America. I am gratified to have him as a mentor for few years. I would like to thank my major advisor Dr. James R. Johnson for his support, technical guidance and invaluable suggestions throughout my doctoral degree which helped me to think maturely and scientifically. I would also like to thank all my committee members, Dr. Hassan Almoazen, Dr. William McLaughlin, Dr. Arthur B. Straughn, and Dr. George C. Wood and for their valuable time, technical inputs and suggestions throughout my work.

I would also like to thank present lab members Mrs. Yinqi Zhou, Mr. Jin Xu, Dr. Frank Zhang, Mrs. Sonia Bedi, Mrs. Rui Zhu and Lou Hao and members from Dr. Wood's group, for their timely help, cooperation and support during laboratory work and to my previous lab member Om Anand for his encouragement.

My special thanks to Dr. Hari R. Desu, Dr. Wen Qu and Mr. Ed Brunson for their valuable suggestions throughout my work that encouraged me further to learn more about the subject. I would like to thank Micromeritics Instruments Corporation for providing digisizer as a gift to University of Tennessee which was a crucial instrument for my project. I would like to thank department of pharmaceutical sciences and University of Tennessee for allowing me to pursue graduate studies.

Last but not least, a very special thanks to my wife Jyothi and without her support I could not finish this long journey of graduate studies. She encouraged me in each and every part of my life during this journey by caring for both me and our loving son Ivan. 


\begin{abstract}
Micronization is one of the common processes for size reduction to increase surface area of poorly soluble Active Pharmaceutical Ingredient's (API). This size reduction improves the dissolution rate and permeability thereby increasing the bioavailability for hydrophobic API's.
\end{abstract}

Tablets and capsules are the most marketed and easy to manufacture solid dosage forms. During manufacturing of tablets, high compression forces are applied uniaxially on the powder bed to get a coherent consolidated compact with good tensile strength. So, diluents are required to mix with API's and compress into tablets. When this mixture is compressed into tablets, there is a possibility of agglomeration of micronized API to API particles together within the tablet due to their high surface area and high surface free energy during compression. Increasing the particle size and decreasing the surface is generally expected to decrease the dissolution rate of the API.

Micronized furosemide and griseofulvin were used as model API's based on the biopharmaceutical classification system to study the effect of compression force on particle agglomeration during compression and also to study the processes and materials to prevent that agglomeration. The size of furosemide and griseofulvin particles was measured after disintegration of tablets compressed by varying compression forces and drug loading. Effect of size of diluents on agglomeration of API's was also studied by varying the size of diluents like lactose mono hydrate and dicalcium phosphate dihydrate. The bonding mechanism for formation of agglomerates of these API's during compression was studied by comparing the tensile strength of the compacts soaked and dried in solvents of various dielectric media. Prevention of micronized particle agglomeration during compression was studied by using particle agglomeration inhibitors like PEG 3350, Lutrol F68 micro, hydroxyl propyl starch polymer and by various treatment methods like physical mixing, hexane slurry method, roller compaction and spray drying. The particles were characterized for change in crystal structure, polymorphism and surface morphology by X-ray diffraction, thermal studies and microscopy studies.

Micronized furosemide and griseofulvin particle size was increased significantly and linearly by increasing the drug loading and compression force during compression. The size of the formed agglomerates was directly proportional to particle size of diluents. The dominating bonding mechanism responsible for agglomerate formation was found to be solid bridge formation between drug particles during compression. The API to API particle agglomeration during compression was successfully prevented by using particle agglomeration inhibitors (PAI's). Treatment with hexane and increase in the level of super disintegrant provided limited prevention of agglomeration during compression for furosemide particles. The spray drying with either mannitol or hydroxy propyl starch for micronized furosemide significantly reduced the agglomeration of API particles during compression. Roller compaction process with Lutrol F68 micro and PEG 3350 provided significant reduction in the agglomeration of drug particles for both the API's during 
compression. Compression force and treatment with PAI's on polymorphism was observed in changing the crystal structure and polymorphism during compression and confirmed by thermal and X-ray crystallographic studies for both the API's. Scanning Electron Microscopy studies revealed compression force and roller compaction process with PAI's changed the surface morphology of both the API's.

The results of the above studies indicated that compression of micronized hydrophobic poorly soluble drugs into tablets affects the dissolution rate due to agglomeration of API. The micronization process for improving the dissolution rate of poorly soluble drugs showed disadvantageous with respect to tablets compressed at high compression forces because of agglomeration of furosemide and griseofulvin particles during compression. Hence control of particle size and selection of size of diluents are necessary during formulation of tablets of hydrophobic poorly soluble drugs. Primary particle size increase of micronized hydrophobic drugs during compression can also be minimized by treatment with particle agglomeration inhibitors prior to compression. 


\section{TABLE OF CONTENTS}

CHAPTER 1. INTRODUCTION ....................................................................................1

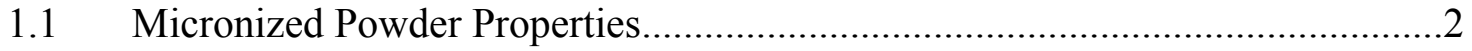

1.2 Mixing of Micronized Powders ......................................................................4

1.3 Compaction of Micronized Powders ............................................................... 4

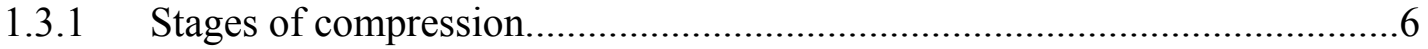

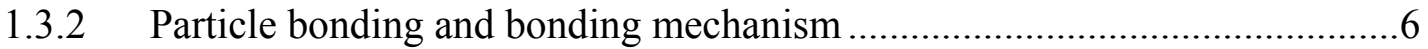

1.3.3 Evaluation of bonding mechanism......................................................... 9

1.4 Agglomeration of Micronized Powders.......................................................... 10

1.5 Particle Agglomeration Inhibitors (PAIs) ............................................. 10

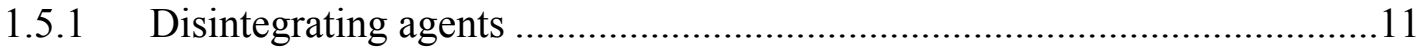

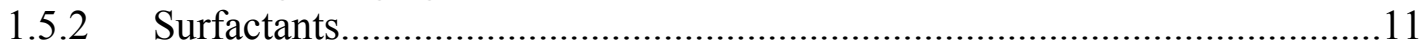

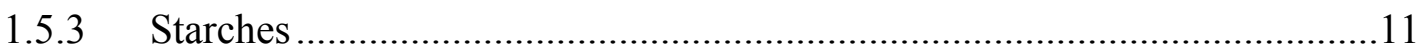

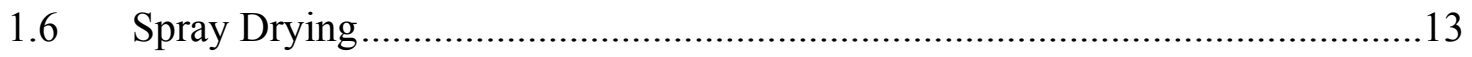

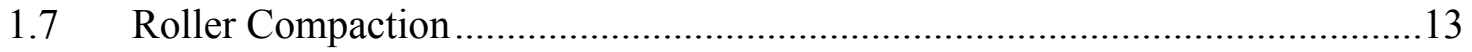

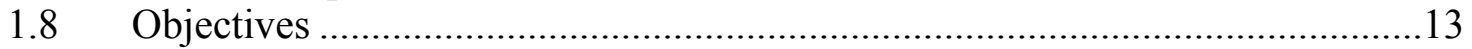

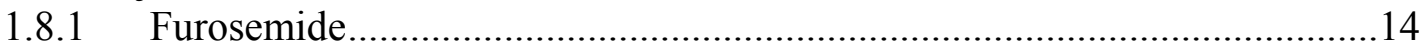

1.8.2 Griseofulvin .................................................................................. 14

CHAPTER 2. COMPRESSION FORCE INFLUENCE ON DRUG-DRUG PARTICLE AGGLOMERATION DURING TABLETING OF MICRONIZED

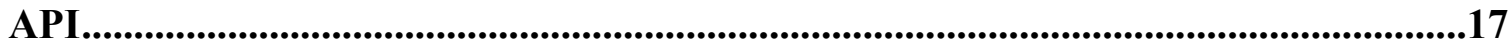

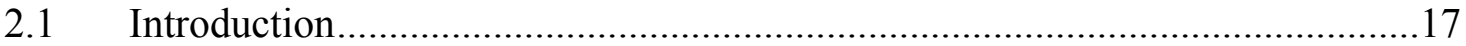

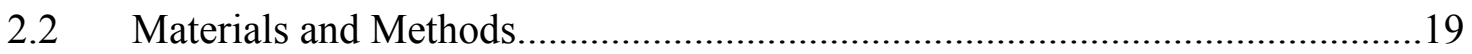

2.2.1 Materials .............................................................................................. 19

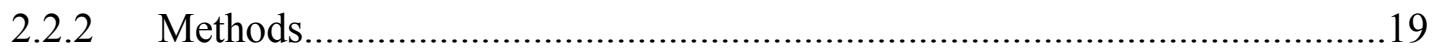

2.2.2.1 Determination of equilibrium solubility studies............................. 19

2.2.2.2 Preparation of saturated solutions..................................................... 19

2.2.2.3 Preparation of blends .................................................................. 20

2.2.2.4 Effect of diluent particle size......................................................... 20

2.2.2.4.1 Estimation of diluents particle size ........................................... 20

2.2.2.4.2 Estimation of diluents specific surface area............................... 20

2.2.2.5 Compression of tablets ................................................................. 20

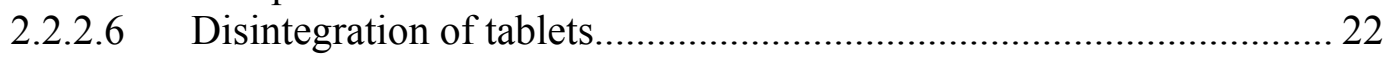

2.2.2.7 Validation of disintegration process ................................................. 22

2.2.2.8 Estimation of drug in the residue..................................................... 22

2.2.2.9 Measurement of particle size ....................................................... 22

2.2.2.10 Modulated differential scanning calorimetry ................................. 23

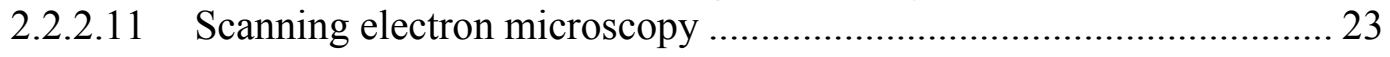

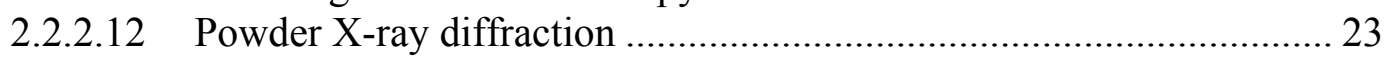

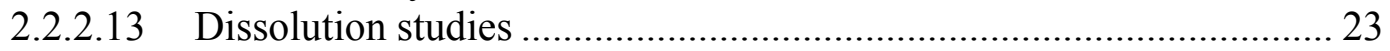

2.2.2.14 Drug-drug bonding mechanism .................................................... 25

2.2.2.14.1 Solubility studies of griseofulvin in organic solvents ................. 25

2.2.2.14.2 Measurement of tensile strength of compacts ........................... 25 


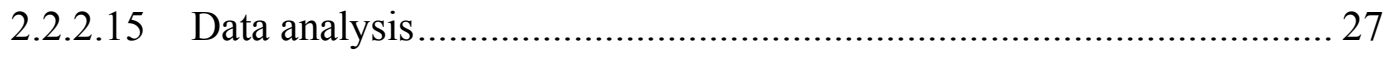

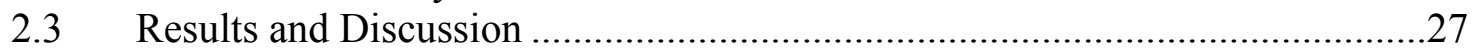

2.3.1 Particle size analysis and SEM characterization.......................................27

2.3.2 Validation of disintegration process and assay of filtered residue ...............30

2.3.3 Effect of compression force and drug loading .............................................30

2.3.4 Modulated differential scanning calorimetry .................................................34

2.3.5 Powder X-ray diffraction studies ................................................................34

2.3.6 Effect of diluent particle size ..................................................................39

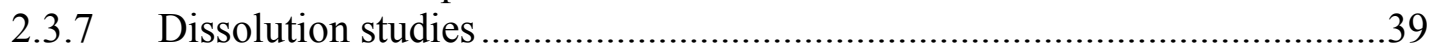

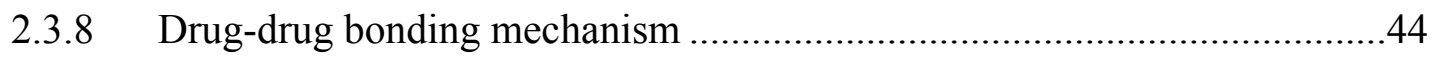

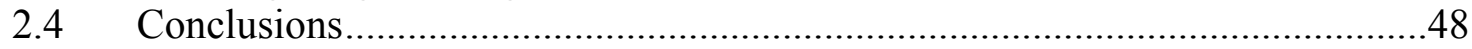

\section{CHAPTER 3. PREVENTION OF DRUG-DRUG PARTICLE
AGGLOMERATION DURING COMPRESSION ..............................................49}

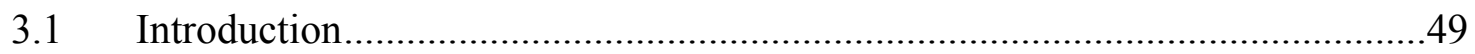

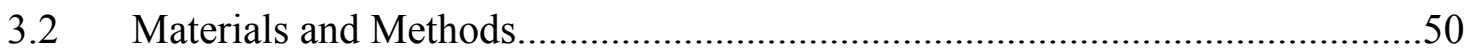

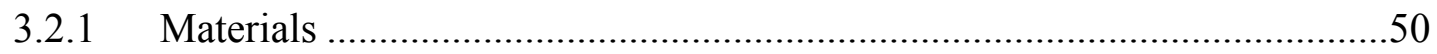

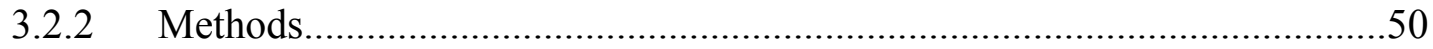

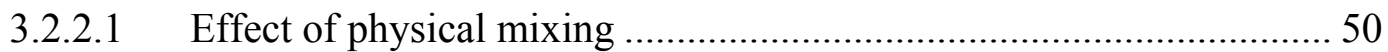

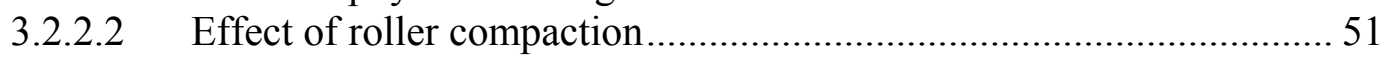

3.2.2.3 Effect of spray drying .................................................................. 51

3.2.2.4 Effect of level of super disintegrant .................................................. 60

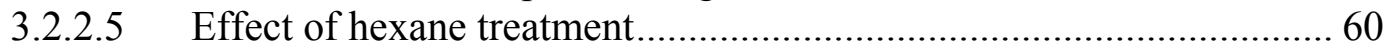

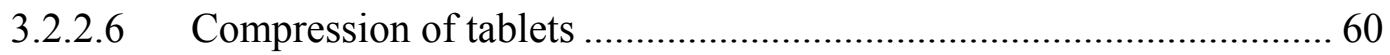

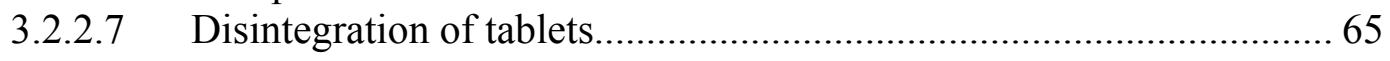

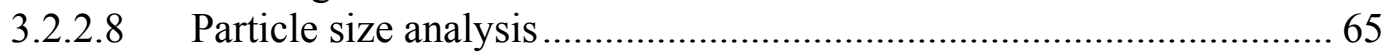

3.2.2.9 Modulated differential scanning calorimetry ........................................ 65

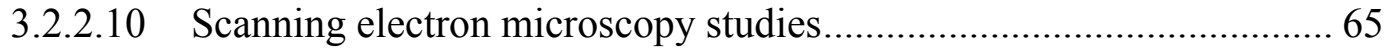

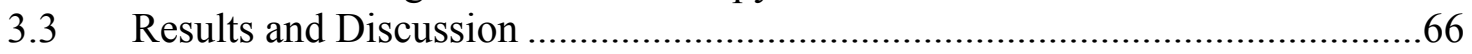

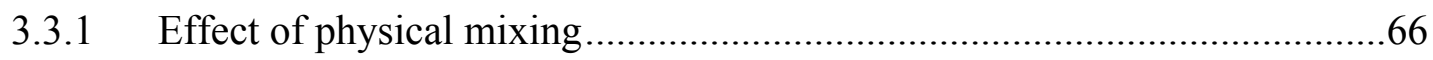

3.3.2 Effect of roller compaction .....................................................................

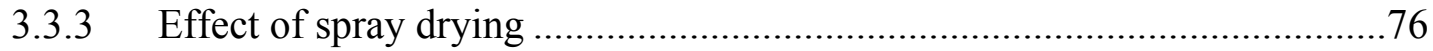

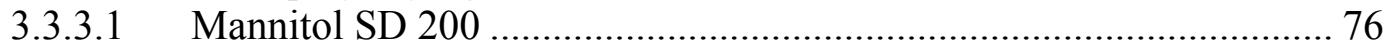

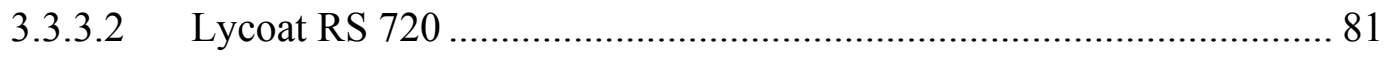

3.3.4 Effect of concentration of super disintegrant..............................................81

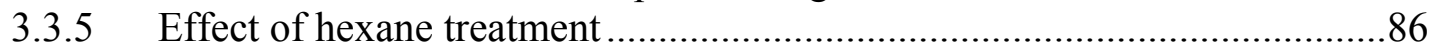

3.3.6 Modulated differential scanning calorimetry ..............................................86

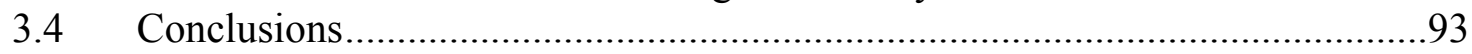

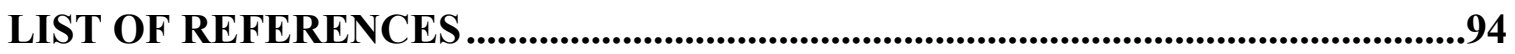

VITA........................................................................................................................................104 


\section{LIST OF TABLES}

Table 1-1. Marketed approaches to solubility enhancement of poorly water- soluble drug substances ..............................................................................................

Table 1-2. Some specifications of bonding mechanisms in compacted powders ............8

Table 2-1. Formulations for furosemide compression force and drug loading study ....21

Table 2-2. Formulations for griseofulvin compression force and drug loading study ...21

Table 2-3. Formulation for diluent particle size effect study .................................21

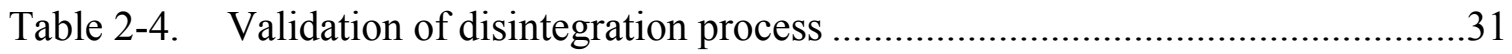

Table 2-5. Powder properties of diluents fractions ..................................................40

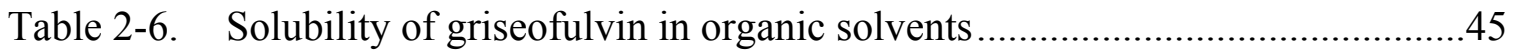

Table 3-1. Formulations for effect of physical mixing on griseofulvin particles...........52

Table 3-2. Formulations for effect of physical mixing on furosemide particles ............53

Table 3-3. Formulations for effect of physical mixing of PEG 3350 on furosemide

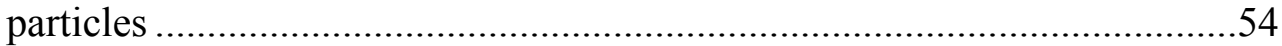

Table 3-4. Formulations for effect of roller compaction on griseofulvin particles ........55

Table 3-5. Formulations for effect of physical mixing and roller compaction on furosemide particles .........................................................................56

Table 3-6. Formulations for effect of physical mixing of mannitol on furosemide particles

Table 3-7. Formulations for effect of spray drying with mannitol on furosemide particles

Table 3-8. Formulations for effect of spray drying with Lycoat RS 720 on furosemide particles

Table 3-9. Formulations for level of super disintegrant

Table 3-10. Formulation for hexane treatment effect for furosemide 


\section{LIST OF FIGURES}

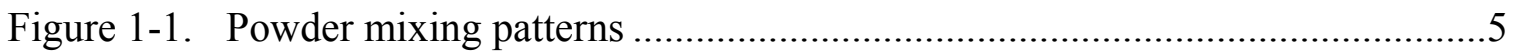

Figure 1-2. Stages of compression........................................................................

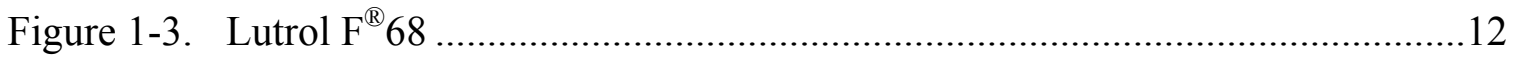

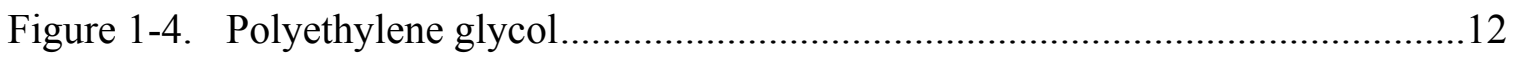

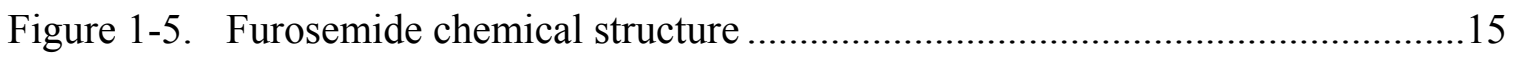

Figure 1-6. Griseofulvin chemical structure ……………………………………….....15

Figure 2-1. Scheme of particles and tablets treatment for characterization ....................24

Figure 2-2. Scheme for API-API bonding study ……………......................................26

Figure 2-3. Particle size distribution of furosemide and griseofulvin API particles .......28

Figure 2-4. SEM images of furosemide and griseofulvin API particles...........................29

Figure 2-5. Effect of compression force and drug loading on furosemide particles .......32

Figure 2-6. Effect of compression force and drug loading on griseofulvin particles .......33

Figure 2-7. MDSC thermograms of furosemide before and after compression ................35

Figure 2-8. MDSC thermograms of griseofulvin before and after compression..............36

Figure 2-9. X-ray powder diffraction of furosemide particles .........................................37

Figure 2-10. X-ray powder diffraction of griseofulvin particles .........................................38

Figure 2-11. Effect of particle size of diluents on furosemide during compression at $2 \mathrm{mT}$ with $30 \%$ drug loading ......................................................................4 41

Figure 2-12. Dissolution of furosemide particles …………………………………........42

Figure 2-13. Dissolution of griseofulvin particles .............................................................43

Figure 2-14. Effect of dielectric constant and tensile strength .........................................46

Figure 2-15. Effect of dielectric constant on remaining tensile strength of compacts ......47

Figure 3-1. Preparation procedure for co-processed powder of mannitol and furosemide 
Figure 3-2. Preparation procedure for co-processed furosemide and Lycoat RS 720

powder

Figure 3-3. Effect of physical mixing with PEG 3350 on griseofulvin particles during compression

Figure 3-4. Effect of physical mixing with Lutrol F68 on griseofulvin particles during compression

Figure 3-5. Effect of physical mixing of Lutrol F68 micro on furosemide particles during compression

Figure 3-6. Effect of ratio of level of Lutrol F68 micro on agglomeration of furosemide particles during compression.

Figure 3-7. SEM image of (a) griseofulvin API particles (b) roller compacted griseofulvin + Lutrol F68 micro (c) roller compacted griseofulvin + PEG 3350 .72

Figure 3-8. Effect of roller compaction with PEG 3350 on griseofulvin particles .73

Figure 3-9. Effect of roller compaction with Lutrol F68 micro on griseofulvin particles

Figure 3-10. Effect of physical mixing and roller compaction with Lutrol F68 micro on furosemide particles during compression.

Figure 3-11. Particle size distribution of furosemide particles (a) before compression (b) after compression at $2 \mathrm{mT}$ with $30 \%$ drug loading

Figure 3-12. SEM image of furosemide particles (a) before compression (b) after compression (F-15) .78

Figure 3-13. Effect of compression force and drug loading furosemide particles with mannitol as a diluent by physical mixing.

Figure 3-14. SEM image of (a) mannitol SD 200 particles (b) mannitol SD $200+$ furosemide after spray drying (c) furosemide particles (d) furosemide particles after compression (F-18)

Figure 3-15. Effect of compression force and drug loading on furosemide particles by spray drying process.

Figure 3-16. SEM image (a) furosemide particles (b) furosemide + Lycoat RS 720 spray dried particles

Figure 3-17. Effect of spray drying of furosemide with Lycoat RS 720 on furosemide particles during compression. 
Figure 3-18. Effect of AC-Di-Sol concentration on furosemide particles during compression.

Figure 3-19. SEM images (a) 400X, (b) 200X, (c) 6400X magnification of furosemide particles coated on lactose monohydrate particle in hexane slurry treatment

Figure 3-20. Effect of treatment with hexane on furosemide particles during compression. .88

Figure 3-21. MDSC thermograms of furosemide particles after treatment with mannitol spray dried.

Figure 3-22. MDSC thermograms of furosemide after particles after treatment with Lycoat RS 720 .....

Figure 3-23. MDSC thermograms of griseofulvin after physical treatment with PEG3350 and Lutrol F68

Figure 3-24. MDSC thermograms of griseofulvin particles after roller compaction with PEG 3350 and Lutrol F68 


\section{LIST OF ABBREVIATIONS}

$\begin{array}{ll}\AA & \text { Angstroms } \\ \lambda & \text { Lambda } \\ \mu & \text { Micron } \\ \text { API } & \text { Active Pharmaceutical Ingredient } \\ \text { BCS } & \text { Biopharmaceutical Classification System } \\ \text { DSC } & \text { Differential Scanning Calorimetry } \\ \text { FFBE } & \text { Flat Face Bevel Edge } \\ \text { FSD } & \text { Furosemide } \\ \text { GSF } & \text { Griseofulvin } \\ \text { IDD } & \text { Insoluble Drug Delivery } \\ \text { MDSC } & \text { Modulated Differential Scanning Calorimetry } \\ \text { nm } & \text { Nanometer } \\ \text { PAI } & \text { Particle Agglomeration Inhibitor } \\ \text { PEG } & \text { Poly Ethylene Glycol } \\ \text { PTFE } & \text { Poly Tetra Fluoro Ethylene } \\ \text { SAS } & \text { Supercritical Anti Solvent } \\ \text { SCF } & \text { Super Critical Fluid } \\ \text { SEDDS } & \text { Self Emulsifying Drug Delivery System } \\ \text { SEM } & \text { Scanning Electron Microscope } \\ \text { SFE-EM } & \text { Supercritical Fluid Extraction Emulsion } \\ \text { SMEDDS } & \text { Self Micro Emulsifying Drug Delivery System } \\ \text { USP } & \text { United States Pharmacopeia } \\ \text { XRPD } & \text { X-Ray Powder Diffraction }\end{array}$




\section{CHAPTER 1. INTRODUCTION}

Poor aqueous solubility and poor oral bioavailability of drugs are the two most discussed topics in the published pharmaceutical research. Most of the new chemical entities synthesized currently through traditional combinatorial chemistry or through analogous synthesis schemes are hydrophobic in nature and are difficult to dissolve in aqueous solutions. The desired pharmacological response for administered drug can be manifested only when it is available in sufficient concentration at receptors. ${ }^{1}$ At present $40 \%$ of the new chemical entities in the developmental pipeline are poorly soluble, and even up to $60 \%$ of the compounds coming directly from synthesis are poorly soluble. ${ }^{2-5}$ Some diseases need chronic drug treatment and other diseases need acute or short term drug therapy. Chronic drug therapy or extended drug treatment can be achieved by modifying drug release characteristics from dosage forms. For acute drug therapy, most of the patients prefer injections, solutions or immediate release solid dosage forms. The oral route is generally the preferred route of drug administration where possible, due to patient compliance, convenience and lower production costs. For any drug to be absorbed into systemic circulation after oral administration that drug should dissolve in the gastric

fluids. ${ }^{6}$ For better therapeutic response the drug must be in molecular dispersion form that means a true solution at absorption site. According to Biopharmaceutical Classification System the drug dissolution and gastrointestinal permeability are the fundamental parameters controlling the bioavailability of drugs. ${ }^{7}$ Absorption of drugs through the intestine is purely depends on their physicochemical properties like molecular weight, $\mathrm{pKa}, \log \mathrm{P}$, solubility, intrinsic dissolution rate, polymorphic nature of drug, particle size, $\mathrm{pH}$ environment of gastro intestinal tract and its content. Based on the aqueous solubility and intestinal permeability the biopharmaceutical classification system classified the drugs into four categories. For any drug substance classified as highly soluble in BCS class I and class III category its highest dose should be soluble in $250 \mathrm{ml}$ or less aqueous medium at $\mathrm{pH}$ range of 1.0-7.5. ${ }^{8}$ Dissolution of a drug in the gastrointestinal fluids is often the rate limiting step for oral bioavailability. According to Noyes-Whitney in Equation 1-1. ${ }^{9}$

$$
\frac{d W}{d t}=\frac{D A\left(C_{s}-C\right)}{h}
$$

Where $\mathrm{dw} / \mathrm{dt}$ is the rate of dissolution of a drug, $\mathrm{D}$ is the diffusion coefficient of a drug, $\mathrm{A}$ is the surface area of a drug accessible to dissolution media, $\mathrm{C}_{\mathrm{s}}$ is the concentration of a drug surrounding layer of a solid, $\mathrm{C}$ is the concentration a drug in the bulk dissolution media which is negligible compared to $\mathrm{C}_{\mathrm{s}}{ }^{10}, \mathrm{~h}$ is the thickness of a diffusion layer. In the above Equation 1-1, the diffusion coefficient D is depends on the viscosity of gastrointestinal fluid and/or its contents and the thickness of a diffusion layer $\mathrm{h}$ depends on the hydrodynamics during gastrointestinal transit. ${ }^{11}$ These latter two parameters have less impact as a target to improve the dissolution rate of poorly soluble drugs. The other two terms in the Equation 1-1 can be used to improve bioavailability by increasing the surface area and by increasing the saturation solubility. Several literature sources discussed saturation solubility increasing with decrease in particle size. ${ }^{12-14}$ 
Dissolution rate increase can be achieved by reducing the particle size of the drug and optimizing the wetting characteristics of compound surface to decrease the diffusion layer thickness and by increasing the apparent solubility of a drug. ${ }^{15}$ Christer. $\mathrm{N}$ et al., studied the milled griseofulvin and found that the increase in solubility was due to increase in surface free energy and reduction in heat of fusion and disordering of solid structure ${ }^{16}$ Currently; there are numerous techniques available to increase the dissolution rate by particle size reduction to micro and nano size, ${ }^{17-20}$ by forming solid dispersions, ${ }^{21,22}$ by forming inclusion complexes with cyclodextrins ${ }^{23,24}$ and by forming salts. ${ }^{25}$ Increase in dissolution rate of poorly soluble drugs by micronization is a wellknown topic. ${ }^{26}$ For some drugs, like griseofulvin the degree of alimentary absorption and increase in blood concentration is directly proportional to particle size. ${ }^{27}$ Instability of solid dispersion systems ${ }^{28}$ and the size of the molecule and its conformational fit for inclusion complexation with cyclodextrin ring are some of the disadvantages ${ }^{29}$ Among these techniques the size reduction to micron or nano size is one of the suitable techniques to get successful enhancement of dissolution rate for poorly soluble drugs. ${ }^{30}$ Currently marketed and under development technologies for poorly water soluble drug substance are presented in Table 1-1. ${ }^{31}$

\subsection{Micronized Powder Properties}

Knowledge about micronized or nano sized powder properties is very important in the pharmaceutical industry. The major and important properties of micronized powders are 1) individual particle shape and surface area, 2) particle size distribution and total surface area. As the dissolution rate of poorly soluble drugs increases with decreasing the particle size, the estimation of particle size is an important material characterization parameter in the drug development stage. Generally the particle size is expressed in microns ( $\mu \mathrm{m}$, micrometer) or nanometer $(\mathrm{nm})$. But for dissolution generally must be related to surface area. The most critical part of particle size analysis is method development and validation. There are several methods in practice across the industry for estimation of particle size. Some practical issues to be considered in estimation particle size, in dry method the particles could be exposed to significant stress as this method uses heavy air streams. Whereas in wet analysis, the material should not be soluble in the dispersant and it should compatible with the wetting agent used in the media. The impact of shear due to sonication during measurement should be evaluated so that the particles should not be fractured.

The micron $\left(10^{-6}\right)$ or nano $\left(10^{-9}\right)$ size particles are very small in size; their surface area will be large because of generation of new surfaces during micronization process. This increase in surface area is responsible for hastening the dissolution rate of poorly soluble drugs. Surface area of powders can be measured normally by Brünauer-EmmettTeller or B.E.T method and expressed as specific surface area. 
Table 1-1. Marketed approaches to solubility enhancement of poorly water- soluble drug substances

\begin{tabular}{|c|c|c|c|c|c|}
\hline Particle size & Method & Process & Technology & Owner & Marketed products \\
\hline \multirow[t]{7}{*}{ Micron (nano particle) } & Down-sizing & Milling & Nanocrystal & Elan & $\begin{array}{c}\text { Rapamune }^{\circledR}, \\
\text { Emend }^{\circledR}\end{array}$ \\
\hline & Down-sizing & Homogenation & IDD & SkyePharma & Triglide $^{\mathrm{TM}}$ \\
\hline & Down-sizing & Homogenation & Nanopure & PharmaSol & Under development \\
\hline & Down-sizing & Homogenation & Nanoedge & Baxter & Under development \\
\hline & Down-sizing & SCF & SCF milling & DuPont & Under development \\
\hline & Build-up & $\mathrm{SCF}$ & SFEE & Ferro Corp & Under development \\
\hline & Build-up & SCF & SAS-EMTM & Thar Tech & Under development \\
\hline
\end{tabular}




\subsection{Mixing of Micronized Powders}

Mixing of micronized powders along with other excipients in drug development is a crucial and cumbersome process. Two types of mixing exist to get good blend homogeneity in solid dosage form development. They are 1) ordered mixing, 2) random mixing. The mixing process is represented in Figure 1-1. Ordered mixture is formed by mixing fine cohesive powders and coarse free flowing powders. Adsorption of fine particles on coarse particles determines the homogeneity of the blend. For any random mixture the particles in the blend need not to be in different size, but the content of the sample taken from the random mixture at any place in the mixture should be same. ${ }^{32,33}$ Particle segregation and agglomeration are the two major problems that could arise during blending of different size powders. With some limitations the ordered mixing process reduces the segregation. This segregation and agglomeration can be minimized by modifying the particle size and the shape of the drug particles. ${ }^{34}$ Type and extent of particle adsorption can be shown to be dependent on the size and physicochemical properties of the particles. For some drugs like antibiotics adsorbs more on sorbitol instant pharma compared to crystalline sorbitol due to its high adsorption capacity and binding strength. ${ }^{35}$ For micronized cohesive hydrophobic drugs like griseofulvin the ordered mixing process by mixing with sodium chloride as diluent increased the contact surface area exposed to dissolution media. ${ }^{36}$ Hydrophobic diluents such as di calcium phosphate dihydrate gave limited increase in the dissolution rate of griseofulvin suggests that use of a soluble diluent is preferred. The higher degree of surface coverage by drug particles on carriers by ordered mixing increased the dissolution rate of griseofulvin blend as well as tablets. This effect was altered by increasing the drug loading and also due to formation of drug film on carrier particles which blocks instantaneous dissolution of the latter. ${ }^{37,38}$

\subsection{Compaction of Micronized Powders}

Particle size importance during compaction of powders was studied over the years. ${ }^{39-41}$ Eighty per cent of the human use and marketed dosage forms are tablets. ${ }^{42}$ Tablets are the most patient compliant, suitable for large scale production and cost effective unit dosage forms in the market. All tablets should possess adequate mechanical strength to with stand processing and transportation and deliver the drug in a predetermined pattern.

The effect of compression force on particle size distribution will provide information about the particles morphology. During the tableting process, the massive compression force applied to the powder bed induces considerable deformation in the solid particles. The expected data from these compression studies should provide whether the particles undergoing any morphological changes during compression like formation of large crystals or aggregates by melting or formation of high energy fine solid particles by fragmentation. For most of the pharmaceutical blends these forces are large enough to exceed elastic limit of the solid that lead to breakage of particles by fracture. 


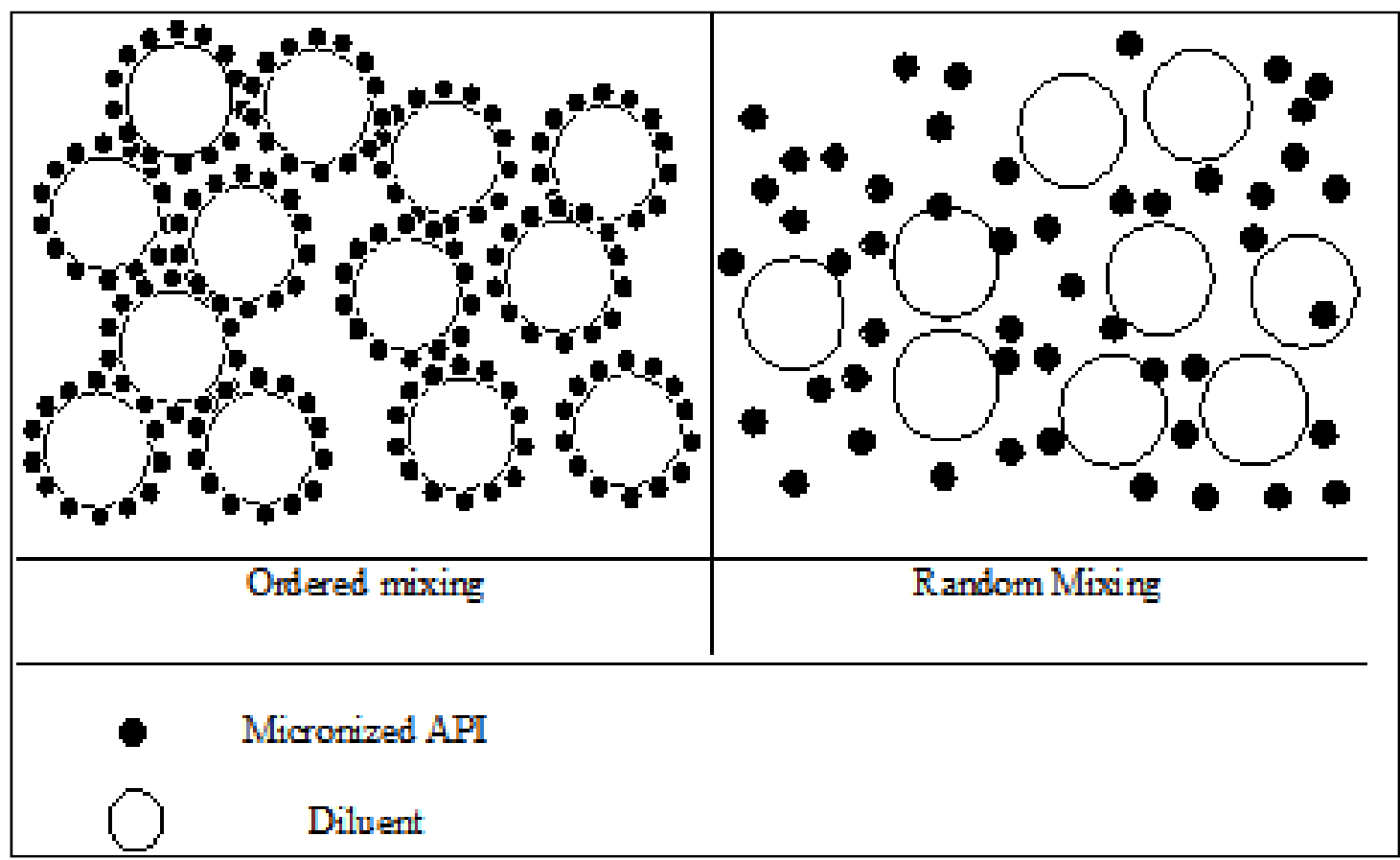

Figure 1-1. Powder mixing patterns 


\subsubsection{Stages of compression}

When an external force is applied uniaxially on a powder bed in a die its volume reduces and its interparticulate porosity decreases. The term compressibility is defined as the ability of a powder material to reduce its volume under pressure, whereas the compactability is the ability of a material to form a solid compact with specific mechanical strength. ${ }^{43}$ Stages of powder compression are presented in Figure 1-2. ${ }^{44}$

An external force applied on a powder bed initially forces the small particles in the powder blend to rearrange by sliding and fill the voids between the particles. Upon further increasing the force there will be a friction between particles that prevents further relative movement of the particles inside the die and powder forced to transform into a denser solid compact. At this stage the powder particles at contact points feel stress and start to deform. If the deformed powder particles regain their original shape upon removal of the force then it is called elastic deformation which is a reversible process. If this deformation is irreversible that is called as plastic deformation. For most of the plastically deformed materials fragmentation occurs further and they break into smaller particles upon increase in applied force. These small particles with new surface increase the compact strength by forming interparticulate bonding due their high surface area and surface energy. In summary one single particle undergo several of these processes during compression and with the other materials can form a coherent compact with the welldefined shape of a tablet. As the compression force increases the specific surface area of the particles increases proportionately to some extent and then reduction in the specific surface area is observed due to agglomeration of fragmented particles. The sequences of events occur during tablet compaction process are: a) particle rearrangement, b) elastic deformation, c) plastic deformation, d) particle fragmentation, e) formation of interparticulate bonds. ${ }^{39,45,46}$

\subsubsection{Particle bonding and bonding mechanism}

The two important factors for compactability of powders are the dominating bond mechanism and the surface distance over which these bonds are active. ${ }^{47}$ The volume reduction during compression is an endothermic process that normally requires more energy to form a compact with high surface area by interparticulate attraction forces. Particle bonding during compression is an exothermal process that releases energy. ${ }^{48}$ Most of the pharmaceutical materials possess poor thermal conductivity and low specific heat. Increase in mechanical strength of a compact by particle/ particle interaction can be defined as consolidation. A number of mechanisms have been proposed for interparticulate bonding during compression a) solid bridges caused by melting and subsequent cooling, b) mechanical interlocking between irregularly shaped particles, c) attractive forces like coulomb forces between charged particles, d) covalent bonds, e) hydrogen bonds and f) van der Waals forces. ${ }^{49,50}$ For simplicity the dominating bonding mechanisms can be limited to three: 1) solid bridges, 2) distance attraction forces, 3) mechanical interlocking. ${ }^{51}$ Some specifications of bonding mechanisms in compacted dry powders are presented in Table 1-2. ${ }^{47}$ 


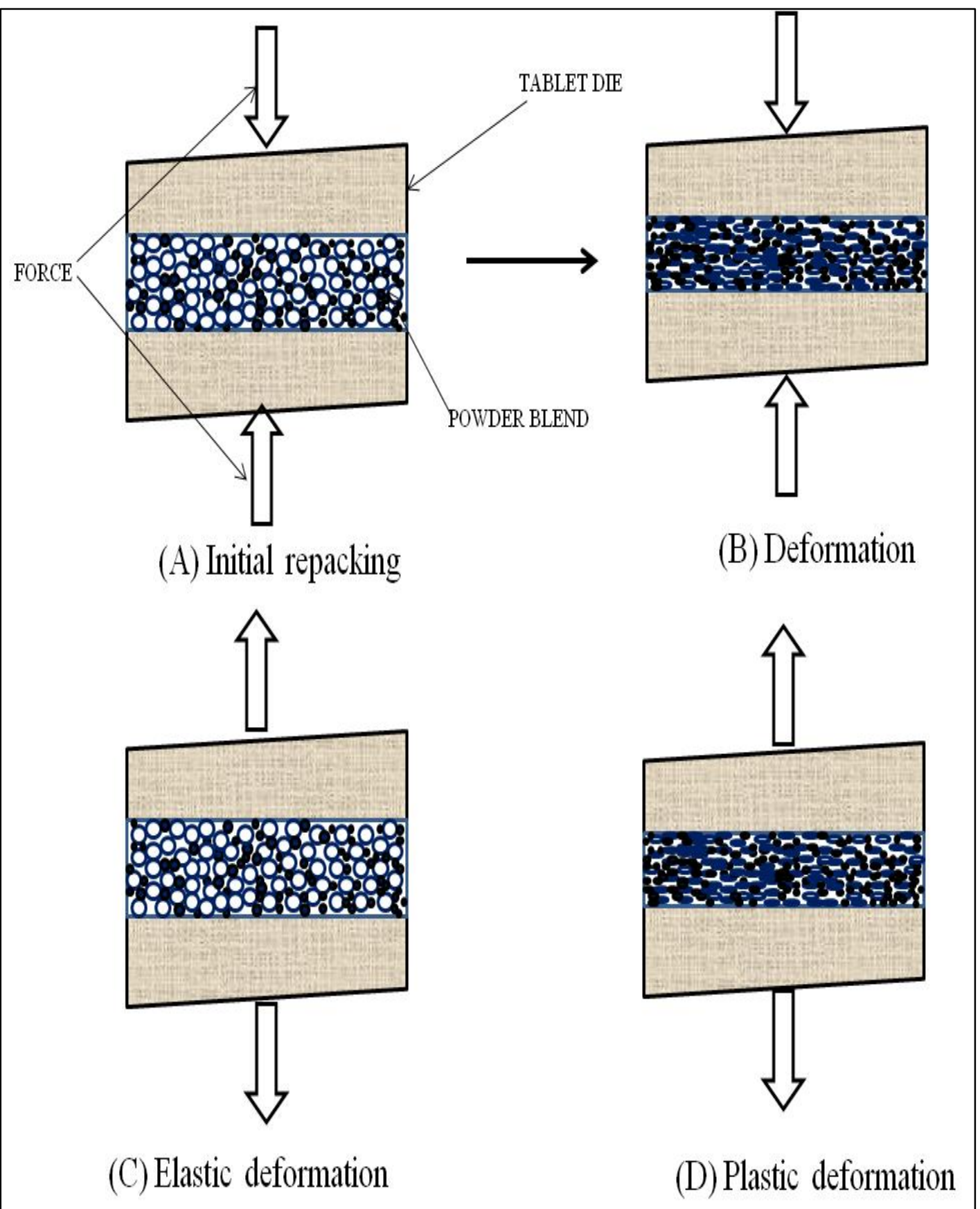

\section{Figure 1-2. Stages of compression}

Modified with permission: Marshall K. 1986. Compression and consolidation of powdered solids. In Leon Lachman HAL, Joseph L. Kanig, editor The Theory and practice of Industrial Pharmacy, 3 ed., Philadelphia: Lea and Febiger. p 66-99. 
Table 1-2. Some specifications of bonding mechanisms in compacted powders

\begin{tabular}{lccc}
\hline $\begin{array}{l}\text { Type } \\
\text { (bonding) }\end{array}$ & $\begin{array}{c}\text { Dissociation energy } \\
(\mathbf{k c a l} / \mathbf{m o l})\end{array}$ & $\begin{array}{c}\text { Separation distance } \\
\text { at equilibrium } \\
(\AA)\end{array}$ & $\begin{array}{c}\text { Maximum } \\
\text { attraction } \\
\text { distance }(\AA)\end{array}$ \\
\hline Solid bridges & $<0-150$ & $<2$ & \\
$\begin{array}{l}\text { Covalent } \\
\text { homopolar }\end{array}$ & $100-200$ & $<2$ & $<10$ \\
$\begin{array}{l}\text { Covalent } \\
\text { heteropolar }\end{array}$ & & & \\
Ionic & $100-200$ & $<3$ &
\end{tabular}

Inter molecular forces

Hydrogen bonding

$2-7$

$3-4$

van der Waals

$1-10$

$3-4$

$100-1000$

Electrostatic

Mechanical Variable depends on the shape, bulkiness and flakiness of the interlocking particles

Å: Angstroms 
During the compression process the particles in direct contact with the upper and lower punch surfaces undergo a pressure effect. Inside the powder bed the particles undergo stress due to particle-to-particle contact. The area at which particle-particle in contact is very small and pressure exerted at contact points can be extremely high. This can raise the temperature and melt the powder particles during compression. For surface fusion there should be formation of localized high temperature hotspots. If the temperature of these hotspots reaches the melting point of the substance being compressed, the surface fusion can occur. ${ }^{52}$ For some pharmaceutical mixtures the excipients could lower the melting point of pure drug during compression, so the surface fusion could occur before reaching the melting point of drug. For some drugs like phenylbutazone and chloropropamide polymorphism could occur during compression and suggests that this mechanochemical effect is due to melting of drug during compression. ${ }^{53}$

\subsubsection{Evaluation of bonding mechanism}

Various bonding mechanisms are involved in the consolidation of powder particles during compression but still it is not clear which bond is dominating in the formation of solid compact with strong mechanical strength. Tensile strength of the compact is the best parameter to assess the dominating bonding acting between the inter particulates. Out of three dominating bonding mechanisms, mechanical interlocking which is twisting or hooking together normally occurs in the particles with elongated and /or irregular in shape. ${ }^{51}$ The above mechanism happens most of the time in compression of metals. The solid bridge formations are assumed to be strong bond formation due to close contact between particles. Whereas the attraction forces between particles are weak and act at a short distance. When the distance between the powder particles reduced to 100$1000 \AA$ the weak long range distance forces, i.e. van der Waals forces, hydrogen bonds and electrostatic attraction forces are likely to occur. ${ }^{54}$

Evaluation of the dominating bonding mechanism responsible for formation of compact was studied by measuring electrical conductance in the compact under pressure, and also by using magnesium stearate as barrier against distance forces between particles. ${ }^{55,56}$ However, most of the pharmaceutical materials are poor conductors of electricity which is prerequisite for conductance. Luangtana-anan et al., described an alternative method to differentiate weak long range distance forces and solid bridge formation during compression. In this method tensile strengths of the compacts were evaluated after soaking the compacts in various liquids with a different dielectric constant. Decrease in the tensile strength results in the weak compacts with increasing dielectric constant of the solvent and reaches to plateau at a dielectric constant of between 10 and 20. ${ }^{49,57-59}$ Van der Waals forces act between most of the particles and it also depends on the properties of the particles and their surrounding medium. In air these bonds dominate in adhesion of the particles. According to Equation 1-2, the energy (W) between two surfaces is directly proportional to the Hamaker constant (A) and indirectly proportional to distance between surfaces (d). 


$$
W=-\frac{A}{12 \pi d^{2}}
$$

The interactive forces between surfaces can be expressed as Equation 1-3.

$$
A_{s / l}=24 \pi d^{2} \gamma_{s / l}
$$

In which $\gamma \mathrm{s} / \mathrm{l}$, is surface energy of particles between two phases of solid and liquid medium. The surface energy of a particle is affected by dielectric constant. A high dielectric constant results in the lower surface energy and thus reduction in the strength of the van der Waals forces.

\subsection{Agglomeration of Micronized Powders}

Even though the micronization process offers advantages among other techniques, it has some limitations as it generates dust, the materials possess poor flow properties which create formulation processing problems. ${ }^{60}$ Due to very small size, and these micronized powders exhibit cohesive and adhesive properties, this result in the formation of agglomerates. These agglomerates may break easily if they formed by weak forces but these may affect the disintegration and dissolution if they are strong. Formation of agglomerates depends on the initial particle size, generally elastic modulus and interparticulate bond strength. Agglomeration of particles is due to inter particulate bonding by weak van der Waals forces. ${ }^{61}$ Strongly agglomerated micronized drugs may not show any improvement in dissolution rate as the exposed external surface area of primary particles to the media is substantially smaller. This shows the disadvantageous effect of micronization. To improve the liquid penetration into the agglomerates and thus to increase the surface area contact use of surfactants are desirable. ${ }^{62-64}$

\subsection{Particle Agglomeration Inhibitors (PAIs)}

Particle agglomeration inhibitors (PAIs) are the agents that prevent the aggregation of API-API particles during compression and also separate the hydrophobic API particles from diluents when in contact with aqueous environment by changing surface properties like increase in wetting and decrease in surface free energy. When micronized hydrophobic API powders were formed into a large solid coherent compacts like tablets their disintegration and dissolution will be decreased due to agglomeration and aggregation. By treating the hydrophobic API with particle separating agent and incorporating that treated particles into a tablet results in improving dissolution rate. The ratio of drug to particle separating agent adjusted to $1: 20$ to about 20:1. ${ }^{65}$ The PAI's should be hydrophilic in nature with low melting point and should also possess good compressible characteristics with high surface area. 


\subsubsection{Disintegrating agents}

Particle agglomeration can be resolved by adding an excess amount of disintegrating agents or combination of agents such as sodium starch glycolate, croscarmellose sodium. As per literature more than $10 \%$ of disintegrating agent can be used for poorly water soluble drug substances to get a desirable dissolution profile. Increase in disintegrant concentration also increases the weight of the tablet. Most disintegrating agents possess capillary and wicking properties for swelling and disintegration of tablets. Some dosage forms show differences in their disintegration and dissolution behavior during shelf life studies due to moisture pick-up by large quantities of disintegrating agents. Sometimes the disintegration process could slow down due to surface coating of disintegrants by micronized hydrophobic API particles or by magnesium stearate. ${ }^{66}$ Some of the more common super disintegrating agents available in the market are Crospovidone (Poly Plasdone ${ }^{\circledR}$ XL-10), croscarmellose sodium (Ac-DiSol $^{\circledR}$ ), and sodium starch glycolate $\left(\right.$ Explotab $\left.^{\circledR}\right)$. Among these super disintegrants croscarmellose sodium improved the disintegration properties from its capillary and swelling properties when used in dry granulation process. A very small percentage $(0.1$ to 1.0) of croscarmellose sodium was used in this thesis because of its insoluble nature in water.

\subsubsection{Surfactants}

Surfactants are agents with hydrophilic and lipophilic groups attached together as head and tail respectively and these surfactants decrease the surface tension between two liquids or between liquid and solid surfaces. Surfactants or wetting agents can be used as particle agglomeration inhibitors. Block co polymers like Lutrol F 68 micronized powder with large surface area can be used as a PAI. The chemical structure of Lutrol F68 is presented in Figure 1-3. The melting point of Lutrol F 68 micro is $52-57^{\circ} \mathrm{C}$. Polyethylene glycols are non ionic substances and can be used as a wetting agent as well as surfactants in preventing particle agglomeration during compression due its low melting and lubricating properties. They also used as lubricants and binders in tablets. The chemical structure of polyethylene glycol is presented in Figure 1-4.Granular PEG 3350 with high surface area and low melting point of $54-58^{\circ} \mathrm{C}$ can be used as PAI. The most important property of PEGs is their solubility in water.

\subsubsection{Starches}

Starch is one of the naturally existing material and best disintegrating agents used in the most tablet formulations. Currently in the market partially gelatinized maize starch $\left(\right.$ Starch $1500^{\circledR}$ ) is available as a binder, disintegrant, flow aid and self-lubricant. Hydroxy propyl starches are hydrophilic and also aid in swelling and dissolution properties of starches. Hydroxy propyl starches are suitable for forming highly transparent films. ${ }^{67}$ 


$$
\begin{aligned}
& \left.\left.\mathrm{HO}-\mathrm{CH}_{2}-\mathrm{CH}_{2}-\mathrm{O}\right]\right]_{\mathrm{a}}\left[\mathrm{CH}_{2}-\stackrel{\mathrm{CH}}{\mathrm{CH}}-\mathrm{O}\right]_{b}\left[\mathrm{CH}_{2}-\mathrm{CH}_{2}-\mathrm{O}\right]_{\mathrm{a}} \mathrm{H} \\
& \sim_{\mathrm{POE}-\mathrm{unit}} \\
& \text { POE-unit } \\
& \text { POP-unit } \\
& \text { POE-unit }
\end{aligned}
$$

Figure 1-3. $\quad$ Lutrol $F^{\circledR} 68$

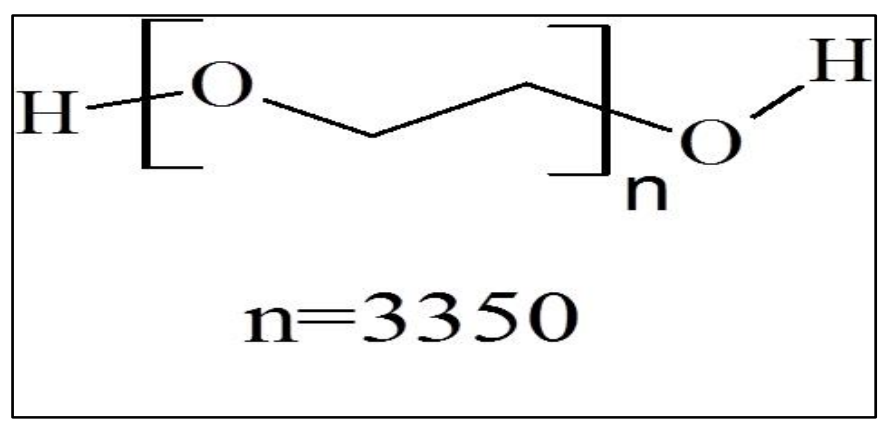

Figure 1-4. Polyethylene glycol 
Dry powder coating consists of spraying a fine powder and plasticizer on particles. ${ }^{68}$ Currently, a hydroxyl propyl starch polymer READILYCOAT ${ }^{\mathrm{TM}}$ is using as an aqueous film coating material for tablets due to its excellent water solubility and rheological properties.

\subsection{Spray Drying}

Spray drying is a process of quench cooling of solution or suspension by atomization through heated air in a drying chamber. Mixing with hot air the atomizer produces small droplets and then rapid evaporation of solvents takes place. The hot air can be same direction or counter-flow. Finally, the formed small fine particles are collected from a cyclone that separates solid particles from hot air. ${ }^{69}$

Co-processing by spray drying for excipients having superior properties compared to individual excipients or their physical mixtures is commonly done. This process gives several advantages like improvement of density, flow, porosity and compactability. Surface energy of materials can be modified by keeping powder bulk properties like solid state and compatibility by spraying drying along with surfactants. ${ }^{70}$ Spray drying of drug substance along with excipients is an alternative method to improve the content uniformity and adsorption of fine cohesive particles on coarse excipients.

\subsection{Roller Compaction}

Dry granulation is one of the granulation processing methods presently most of the industry showing interest due to availability of directly compressible excipients. Still less than 20 percent of API's are not directly compressible due their high dose, poor flow, poor lubrication or highly cohesive properties.

Even with significant disadvantages such as poor tensile strength for tablets produced from roller compacted granules and a relatively expensive process, formulators and industry often prefer the roller compaction process due to its efficient production output, continuous process and less labor intensive operations. The main advantages of the roller compaction process are: 1) increase in bulk density, 2) increase in drug homogeneity by decreasing segregation, 3) increase in disintegration time, and 4) increase in flow of granules by increasing the size of the granules. However, for high drug loading formulations a wet granulation process is often the only practical method. ${ }^{71}$

\section{$1.8 \quad$ Objectives}

The main objective was to estimate the API particle size distribution after compression into tablets of a micronized API. Specific aims included-

- To estimate the effect of compression force on API to API particle agglomeration during compression 
- To estimate the effect of amount of API content on API to API particle agglomeration during compression

- To evaluate the bonding mechanism responsible for agglomeration of API to API during compression

- To prevent the API to API agglomeration during compression by various treatment and processing methods

Based on the Biopharmaceutical Classification System (BCS) two model drugs were selected. BCS class II (poorly soluble and highly permeable) drug griseofulvin and BCS class IV (poorly soluble and poorly permeable) drug furosemide were selected.

\subsubsection{Furosemide}

Chemically furosemide is 5-(aminosulfonyl)-4-chloro-2[(2-furanylmethyl) amino] benzoic acid with a molecular weight of 330.7. Furosemide is light yellowish crystalline powder and it is practically insoluble in water with $206^{\circ} \mathrm{C}$ as melting point. In studies of dissolution rate improvement for furosemide, seven polymorphs were reported, but four of them were found to be true polymorphs. There were also two solvates (DMS and Dioxane) and one amorphous form. ${ }^{72-76}$ There were no reports of polymorph dependant bioavailability of furosemide. Furosemide aqueous solubility at room temperature was reported to be as $0.01825 \mathrm{mg} / \mathrm{ml}$ at room temperature. ${ }^{77}$ Furosemide is a weak acid with 3.8 as an acidic pKa value. ${ }^{78}$ Furosemide was the first high ceiling loop diuretic drug and to also be used as an antihypertensive. Furosemide USP (marketed as Lasix $®$ ) is available in 20, 40 and $80 \mathrm{mg}$ tablets. Furosemide oral solution and its sodium salt as parenteral injection formulations and aerosol formulations are also available in the market. Oral bioavailability of furosemide was reported as approximately $60 \%$ with an elimination half life of $1.5 \mathrm{hrs}$ and a renal elimination of $65 \%$ as intact drug. Furosemide we used in this dissertation was micronized one with mean particle size of around 5 micron and its structure is presented in Figure 1-5.

\subsubsection{Griseofulvin}

Griseofulvin is an antifungal antibiotic discovered in 1959. Chemically griseofulvin is (2S,6'R)-7-chloro-2',4,6-trimethoxy-6'-methyl-3H-spiro[1-benzofuran2,1'-cyclohexan]-2'-ene-3,4'-dione and its molecular weight is 352.77 and its structure is presented in Figure 1-6. It is a white crystalline octahedra and rhombus shape with $219^{\circ} \mathrm{C}$ as melting point. Griseofulvin was classified as a BCS class II category due to its poor solubility and high intestinal permeability. The aqueous solubility of griseofulvin was reported to be $0.03056 \mathrm{mg} / \mathrm{ml}$ and $\log \mathrm{P}$ value of 2.18 . Due to its high variability through oral absorption, griseofulvin is often used as a model drug for any solubility improvement, dissolution improvement, bioavailability improvement and novel dosage forms like SMEDDS/SEDDS. ${ }^{80}$ Griseofulvin is available in micro size and ultra micro size as tablets. GRIS ${ }^{\circledR}$ PEG $125 \mathrm{mg}$ tablets are available as PEG solid dispersion dosage form. In humans this product showed variability when administered with and without 


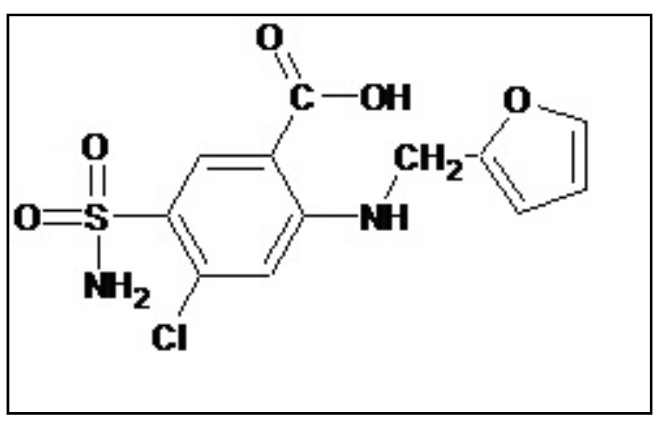

Figure 1-5. Furosemide chemical structure

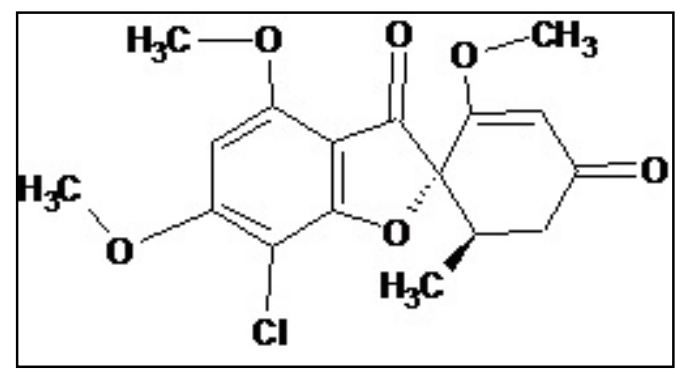

Figure 1-6. Griseofulvin chemical structure 
food. Griseofulvin USP available in tablets, capsules, suspensions, powders. Oral absorption of griseofulvin was greater with foods containing fatty acids. Oral absorption of micro size griseofulvin formulations was variable (20-75\%) whereas ultramicrosize showed $100 \%$ absorption when administered orally. The elimination half-life of griseofulvin found to be $24 \mathrm{hrs}$. By ultra micronization process the oral dose of $500 \mathrm{mg}$ was reduced to $250 \mathrm{mg}$ and proved to be bioequivalent with single $250 \mathrm{mg}$ dose. ${ }^{81,82}$ The particle size of griseofulvin we used in this disseration was around 13 micron in size. 


\section{CHAPTER 2. COMPRESSION FORCE INFLUENCE ON DRUG-DRUG PARTICLE AGGLOMERATION DURING TABLETING OF MICRONIZED}

API

\subsection{Introduction}

Disintegration of tablet followed by dissolution of primary particles of it and absorption of dissolved drug, all these events occur in a sequence after oral administration. ${ }^{83}$ According to Noyes- Whitney equation the dissolution rate is directly proportional to surface area of dissolving drug particles, solubility of the drug in the solvent and indirectly proportional to thickness of the diffusion layer surrounding the solid particles. ${ }^{9}$ For increasing the dissolution rate of hydrophobic drugs, particle size reduction to micron or nano size is a common practice. This size reduction increases the surface area of the drug particles thereby increasing the dissolution rate. Size reduction is a potential advantage. However, compressing micronized drug particles i.e., bringing all these micronized drug particles together along with excipients to form a solid tablet could generate some reduction in its potential advantage. Compared to specific surface area of the drug particles, the effective surface area of the drug that is exposed to gastrointestinal fluids in a dosage form after disintegration is the most important factor to be taken into consideration in development of dosage form. ${ }^{84}$

However, micronization of API for increasing the surface area thereby increasing dissolution rate may not be adequate if the drug undergo agglomeration due to bonding during compression into tablets. When micronized hydrophobic drug powders are diluted with various tableting ingredients and compressed into tablets, the primary particle size of the micronized drug powder can increase significantly during compression. However, to evaluate this effect an intact tablet dissolution does not provide information on what is happening to the API particle size during compression of tablets. ${ }^{85}$

Carless et al. described as coarser particles undergo fragmentation during compression and fill the voids whereas finer particles during compression cannot break further due to their dimensions and hence fuse together. At high compression forces fine particles of size less than 100 microns experience high degree of shear on their surface and at contact points. This results in the local melting of the surfaces below its melting points. Upon releasing the compression force from the powder bed the aspertically molten surfaces solidify and forms sold bridge between particles. A larger surface area of micronized API particles provides a larger number of contact points and hence bonding between the drug particles may occur during compression. As the particle size decreases the tensile strength of the tablets increases; this is due to strong bonding between fine particles ${ }^{86}$ Higuchi et al. described as the compression pressure increases specific surface area initially increases and then decreases..$^{87}$ Due to variation in the distribution of pressure during compression inside the die the resulting compact will be nonhomogeneous. Also defects on the crystal surface or disorders in the lattice due to compaction are known to give rise to structure-sensitive diffusion of the solvent used. ${ }^{88-90}$ Mikko et al. studied the crystal defects during compression for tolbutamide and 
chloropropamide tablets that affects the dissolution rate by grazing incidence $\mathrm{x}$-ray diffraction method. ${ }^{91}$ This increase can significantly alter the dissolution rate of the drug and reduce the absorption for certain drugs.

Khan et al. studied the effect of compaction on particle size of different type of materials (brittle materials, deforming materials and ductile materials with low melting point) and found considerably different particle size after disintegration. ${ }^{92}$ As compaction pressure increases the dissolution rate also increases due to fragmentation of drug particles within in the compact. ${ }^{93}$ However, Hirshorn et al. found a decrease in drug dissolution rate by increasing the compaction force and this could be due to changes in surface area of drug particles after compression and that it also depends on the dilution of drug particles with additives. ${ }^{94}$ In this chapter, the effect of drug loading and compression force on micronized furosemide and griseofulvin API were studied.

Tuladhar et al. described the change in the API particle size during compression was affected by nature of the diluents present in the formulation and also found that the agglomeration of fine particles during compression was independent of rate compression. ${ }^{95}$ Highly homogenous blend for highly cohesive micronized API can be achieved by blending the formulation with highly porous and freely flowing diluents. However, some diluents with highly indented surface may entrap the micronized drug and forms pockets of agglomerates which hamper the dissolution. ${ }^{96,97}$ Effect of particle size of diluent (lactose, di calcium phosphate dihydrate) on micronized API agglomeration during compression were investigated and results were discussed in this chapter.

Changes in the particle size of API during compression might be due to several mechanisms. Solid bridge formation, weak long range forces and mechanical interlocking are the probable mechanisms for compact formation. The strongest bond among the probable mechanism in bond formation, Solid bridges, described as areas in which particles are partially fused during compression by formation of continuous phase between two particles. It is very difficult to define the area responsible for agglomeration of particles during compression if the particles undergo weak long range forces. ${ }^{47,98,99}$ Evaluation of mechanism responsible for agglomeration during compression can be done by filtering out weak long range distance forces. Luangtana-anan and Fell described the evaluation of bonding mechanism method by measuring the tensile strength of compacts after soaking in various liquids with different dielectric constants. The assumption was that increase in dielectric constant liquid media should decrease the tensile strength of the compacts if there is any weak long range distance forces are acting between the particles during soaking. ${ }^{49}$

The objective of the present study was to investigate the effect of compression force, drug loading and effect of diluent particle size on micronized API's: Furosemide and griseofulvin. Particle size distribution, dissolution and characterization of particles by differential scanning calorimetry powder x-ray diffraction and scanning electron microscopy studies were performed. The dominating bond responsible for agglomeration of micronized griseofulvin during compression was evaluated. 


\subsection{Materials and Methods}

\subsubsection{Materials}

Furosemide (micronized API) was purchased from Teva Pharmaceuticals, Israel. Micronized griseofulvin was a generous gift from Wuhan Yuancheng Technology, China. Modified spray dried lactose 315 (Foremost ${ }^{\mathbb{B}} \mathrm{NF}$ Lactose) was purchased from Foremost Forms, Baraboo, WI. Di calcium phosphate di-hydrate $\left(\mathrm{Di}-\mathrm{Tab}^{\mathbb{B}}\right)$ was purchased from Rhodia, Inc, Cranbury, NJ. Croscarmellose sodium (Ac-Di-Sol ${ }^{\circledR}$ ) was obtained from FMC biopolymer, Philadelphia. Magnesium stearate NF was purchased from Mallinckrodt, St. Louis, MO. Pure n-butanol, ethanol and n-hexane were purchased from Sigma Aldrich, St. Louis, MO. ACS grade Acetic acid glacial was purchased from EMD chemicals, Billerica, MA. Sodium dodecyl sulfate and sodium acetate were purchased from Sigma Aldrich, St. Louis, MO.

\subsubsection{Methods}

\subsubsection{Determination of equilibrium solubility studies}

Furosemide and griseofulvin were separately added slowly in excess amount to stirring water and continued stirring in a shaker bath for 24 hours at $37^{\circ} \mathrm{C}$ temperature to achieve equilibrium solubility. Samples in triplicate were withdrawn and filtered using $25 \mathrm{~mm}$ PVDF $0.45 \mu \mathrm{m}$ syringe filter (Millipore Millex) and diluted with water. The diluted samples were assayed for furosemide and griseofulvin by UV-Visible spectroscopy at $272 \mathrm{~nm}$ and $295 \mathrm{~nm}$ respectively. The same procedure was also repeated in acetate buffer USP ( $\mathrm{pH} 4.5)$ for furosemide.

\subsubsection{Preparation of saturated solutions}

Saturated drug solution of furosemide and griseofulvin were prepared by adding excess amount of drug in distilled water with $0.1 \%$ Tween- 80 as a wetting agent and stirred for $12 \mathrm{hrs}$ and vacuum filtered through $0.45 \mu \mathrm{m}$ membrane filter. This saturated drug solution was used as a medium for disintegration of tablets and for the measurement of particle size by suspension method using light scattering technology.

For diluent particle size effect study a saturated furosemide solution was prepared in $0.1 \mathrm{~N}$ hydrochloric acid. This saturated solution was used for disintegration of tablets containing DiTab as diluent to dissolve dicalcium phosphate dihydrate during disintegration. 


\subsubsection{Preparation of blends}

Furosemide and griseofulvin were accurately weighed according to formulation in Tables 2-1 and 2-2. Initially API's and spray dried lactose were mixed together by geometric mixing by passing through \#60 stainless steel mesh twice and finally Ac-DiSol was blended in a plastic bag for $5 \mathrm{~min}$.

\subsubsection{Effect of diluent particle size}

Two diluents were selected for this study. Manufactured supplied spray dried lactose (Foremost Lactose $315 \mathrm{NF}$ ) and dicalcium phosphate dihydrate (DiTab) was fractioned into two fractions by sifting through stainless steel mesh \#60 $(250 \mu \mathrm{m}), \# 80$ $(180 \mu \mathrm{m})$ and $\# 200(75 \mu \mathrm{m})$. Particles from both the diluents under $75 \mu \mathrm{m}$ were collected and named as fraction- 1 and particles under $250 \mu \mathrm{m}$ and above $180 \mu \mathrm{m}$ were also collected and named as fraction-2. Micronized furosemide was used as a model drug for this study. Micronized furosemide and above fractions were accurately weighed according to Table 2-3 and geometrically mixed by passing through \#60 mesh twice and blended with Ac-Di-sol for 5 minutes.

\subsection{Estimation of diluents particle size}

The mean particle sizes of above fractions were confirmed by laser diffraction technique using a Digisizer (Saturn Digisizer ${ }^{\circledR}$ 5200, Micromeritics). Iso propyl alcohol and water were used as a suspending media for measuring particle size of lactose and DiTab respectively. A saturated solution of lactose in isopropyl alcohol and dicalcium phosphate dehydrate (DiTab) in water were prepared by adding excess amounts into these solvents and stirred overnight and vacuum filtered.

\subsection{Estimation of diluents specific surface area}

The specific surface area of above fractions was estimated using gas adsorption technique by Micromeritics Gemini 2360 surface area analyzer. The sample tubes containing sample were dried under stream of $\mathrm{N}_{2}$ gas at $60^{\circ} \mathrm{C}$ using Flowprep 060 (Micromeritics). The specific surface area was calculated using multi point BET equation.

\subsubsection{Compression of tablets}

Tablets of weight $200 \mathrm{mg}$ for furosemide and $400 \mathrm{mg}$ for griseofulvin were compressed on a hydraulic manual bench top laboratory press (Model C, Carver, Inc) with dual scale gauge. 5/16" FFBE (flat face bevel edge) for furosemide and 3/8" FF (flat face) for griseofulvin tooling were used with $7.93 \mathrm{~mm}$ and $9.5 \mathrm{~mm}$ diameter dies 
Table 2-1. Formulations for furosemide compression force and drug loading study

\begin{tabular}{lccc}
\hline Formula & F-1 & F-2 & F-3 \\
\hline Furosemide (\%) & 10.00 & 20.00 & 30.00 \\
Spray dried lactose (\%) & 89.90 & 79.90 & 69.90 \\
Ac-Di-Sol (\%) & 0.10 & 0.10 & 0.10 \\
Total (\%) & 100.00 & 100.00 & 100.00 \\
\hline
\end{tabular}

Table 2-2. Formulations for griseofulvin compression force and drug loading study

\begin{tabular}{lccc}
\hline Formula & G-1 & G-2 & G-3 \\
\hline Griseofulvin (\%) & 15.00 & 30.00 & 60.00 \\
Spray dried lactose (\%) & 84.90 & 69.90 & 39.90 \\
Ac-Di-Sol (\%) & 0.10 & 0.10 & 0.10 \\
Total (\%) & 100.00 & 100.00 & 100.00 \\
\hline
\end{tabular}

Table 2-3. Formulation for diluent particle size effect study

\begin{tabular}{lcc}
\hline Formula & D-1 & D-2 \\
\hline Furosemide (\%) & 30.00 & 30.00 \\
Spray dried lactose (\%) & 69.90 & - \\
DiTab (\%) & - & 69.90 \\
Ac-Di-Sol (\%) & 0.10 & 0.10 \\
Total (\%) & 100.00 & 100.00 \\
\hline
\end{tabular}


respectively. Magnesium stearate was applied to surface of both punches before compression and $3 \mathrm{sec}$ dwell time was maintained for all the compression forces. Four compression forces $0.5 \mathrm{mT}, 1.0 \mathrm{mT}, 1.5 \mathrm{mT}$ and $2.0 \mathrm{mT}$ were studied. Six tablets were compressed for each compression force and each percent drug loading.

For diluents particle size effect study furosemide tablets of $200 \mathrm{mg}$ weight from each diluents blend were compressed at $2 \mathrm{mT}$ compression force using 5/16" FFBE tooling. $3 \mathrm{sec}$ dwell time was also maintained.

\subsubsection{Disintegration of tablets}

Disintegration of tablets was done in QC-21 ${ }^{\mathrm{TM}}$ (Hanson research) disintegration test system in saturated drug solution at room temperature. After disintegration of all six tablets process was further continued for another one more hour to dissolve all soluble excipients in the media. The residue was collected by vacuum filtration. The dried residue was assayed for API and further for particle size measurement and particle characterization and dissolution studies. For the diluents particle size effect study the tablets were also disintegrated as described above.

\subsubsection{Validation of disintegration process}

Disintegration process was validated by measuring the API content in the residue at different intervals of time. Six tablets at $2 \mathrm{mT}$ compression force with $30 \%$ drug loading for each time interval were disintegrated and the residue was filtered at $0.5,1.0$ and $2 \mathrm{hr}$ using vacuum filtration unit. A known quantity of residue was dissolved in methanol and diluted further with methanol and absorbance was measured at $\lambda_{\max }$ of 272 $\mathrm{nm}$ and $294 \mathrm{~nm}$ for furosemide and griseofulvin respectively.

\subsubsection{Estimation of drug in the residue}

The dried residue after filtration was analyzed for pure API's (furosemide or griseofulvin) after disintegration. To ensure all soluble diluents dissolved completely after disintegration. A known quantity of residue was weighed in triplicate and dissolved in methanol and filtered through syringe filter (Acrodisc CR PTFE $0.2 \mu \mathrm{m}, 25 \mathrm{~mm}$ ) and diluted further with methanol. The absorbance was measured using UV/Vis spectrophotometer (Lambda 20, Perkin Elmer) at $\lambda_{\max }$ of $272 \mathrm{~nm}$ and $294 \mathrm{~nm}$ for furosemide and griseofulvin respectively.

\subsubsection{Measurement of particle size}

A Saturn Digisizer ${ }^{\circledR}$ 5200, a high resolution laser particle size analyzer, was used to measure the particle size of the residue from the disintegration study above. Saturated 
furosemide/griseofulvin solution was used as dispersion media to measure the particle size. A suspension was prepared in saturated drug solution to measure the particle size of the drug residue obtained after disintegration. A homogenous liquid-solid bubble free suspension system was maintained by circulating the dispersed system at optimized speed to get a concentration with appropriate obscuration of laser light. Figure 2-1 presents the treatment of particles and tablets for particle size analysis and characterization.

\subsubsection{Modulated differential scanning calorimetry}

Modulated Differential Scanning Calorimetric (MDSC) studies were carried out to confirm the polymorphic changes in furosemide or griseofulvin during processing and tablet compression using a Q2000 DSC (TA instruments).MDSC studies were performed with Q200 Differential Scanning Calorimeter (TA Instruments). Samples of approximately $10 \mathrm{mg}$ were heated in hermetic Tzero sealed aluminum pans. Melting temperature $\left(\mathrm{T}_{\mathrm{m}}\right)$ was measured under $\mathrm{N}_{2}$ atmosphere at flow rate of $50 \mathrm{~mL} / \mathrm{min}$. The heating temperature range was from $25-220^{\circ} \mathrm{C}$ and ramping rate was $3^{\circ} \mathrm{C} / \mathrm{min}$ and heating modulation was $1{ }^{\circ} \mathrm{C} / \mathrm{min}$. The data were analyzed using Universal Analysis software.

\subsubsection{Scanning electron microscopy}

Morphology of particles after compression was studied using an environmental scanning electron microscope. Powder samples of furosemide and griseofulvin were spread onto a double-sided adhesive carbon tape on an aluminum stub. Excess powder was removed by gently tapping the stub. A thin coating $(\sim 25 \mathrm{~nm})$ of gold was applied on to the sample using a Hummer VII Sputtering System and observed under ESEM XL 30 (FEI). API powder samples and samples after compression at $2 \mathrm{mT}$ compression with $30 \%$ drug loading were observed under SEM.

\subsubsection{Powder X-ray diffraction}

Powder X-ray diffraction studies were conducted on a X-ray Diffractomer (Bruker D8 Advance XRD system). The data were collected in the continuous scan mode using a step size of $0.04^{\circ}(2 \theta)$. The scanned range was $4-40^{\circ}(2 \theta)$. Furosemide and griseofulvin particles before and after compression at $2 \mathrm{mT}$ compression force and $30 \%$ drug loading were examined for any changes in crystallography.

\subsubsection{Dissolution studies}

Dissolution studies were conducted for both the API's particles before and after compression. A rate limiting acetate buffer at $\mathrm{pH} 4.5$ medium was used for furosemide particles dissolution and water containing $0.54 \%$ SDS for griseofulvin. A bathless dissolution system from Distek inc operated at $50 \mathrm{rpm}$ paddle speed for furosemide, and 


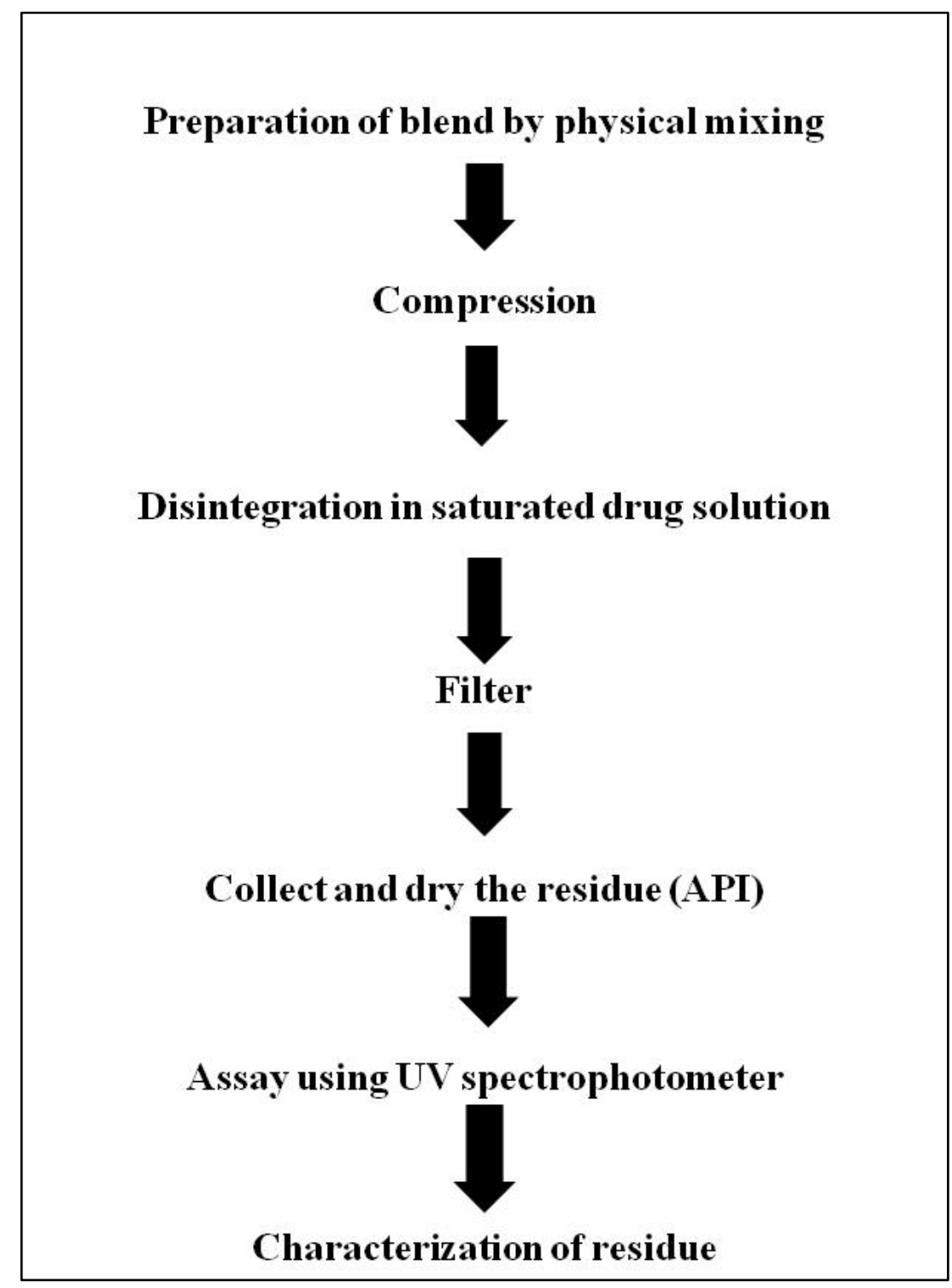

Figure 2-1. Scheme of particles and tablets treatment for characterization 
$75 \mathrm{rpm}$ for griseofulvin at $37^{\circ} \mathrm{C}$ temperature. $20 \mathrm{mg}$ and $15 \mathrm{mg}$ powder samples of furosemide and griseofulvin before and after compression respectively were suspended in $5 \mathrm{ml}$ of dissolution medium and dispensed into dissolution vessels quickly to wet the hydrophobic particles. On line dissolution data was collected at each $5 \mathrm{~min}$ intervals from an automated fibre optic multi channel probes with Indigo software from pION Inc. The absorbance data for furosemide and griseofulvin were collected at $\lambda_{\max }$ of $272 \mathrm{~nm}$ and $294 \mathrm{~nm}$ respectively.

\subsubsection{Drug-drug bonding mechanism}

\subsection{Solubility studies of griseofulvin in organic solvents}

Solubility studies were conducted by adding excess quantity of griseofulvin in hexane, n-butanol and ethanol solvents and stirred overnight. Individual samples were withdrawn and filtered through syringe filter (Acrodisc CR PTFE $0.2 \mu \mathrm{m}, 25 \mathrm{~mm}$ ) and diluted. The concentration of griseofulvin in the organic solvents was measured by UV/Vis spectrophotometer (Lambda 20, Perkin Elmer) at $\lambda_{\max }$ of $294 \mathrm{~nm}$.

\subsection{Measurement of tensile strength of compacts}

Griseofulvin API alone was compressed into compacts using a hydraulic manual bench top tablet press (Model C, Carver Inc) with width of $3 \mathrm{~mm}$ and $9.5 \mathrm{~mm}$ diameter by using $3 / 8$ " flat face tooling. Tablets of griseofulvin were compacted at four different forces $0.5,1.0,1.5$ and $2.0 \mathrm{mT}$. The upper and lower punch faces were lubricated by applying to magnesium stearate (suspension in ethanol) for each compact. For each compression force five tablets were compressed and soaked for $24 \mathrm{hrs}$ in a solvent media pre saturated with griseofulvin. The soaked compacts were dried in desiccator for another $24 \mathrm{hrs}$ before mesuring the tensile strength. For control the compacts were compressd in air/vacuum in the same manner and stored for $24 \mathrm{hrs}$ in dessicator and measured the tensile strength. Solubility of drug in these solvents was also studied. Tensile strength $(\sigma)$ of the dried tablets were derived from the crushing strength (hardness), thickness and diameter of the tablets according to Fell and Newton described in the Equation 2-1

$$
\sigma=\frac{2 F}{10^{6} \pi D T}
$$

Where $\mathrm{F}$ is force required to break the tablet diametrically, $\mathrm{D}$ is diameter of the tablet and $\mathrm{T}$ is thickness of the tablet. The scheme of bonding study is presented in Figure 2-2. 


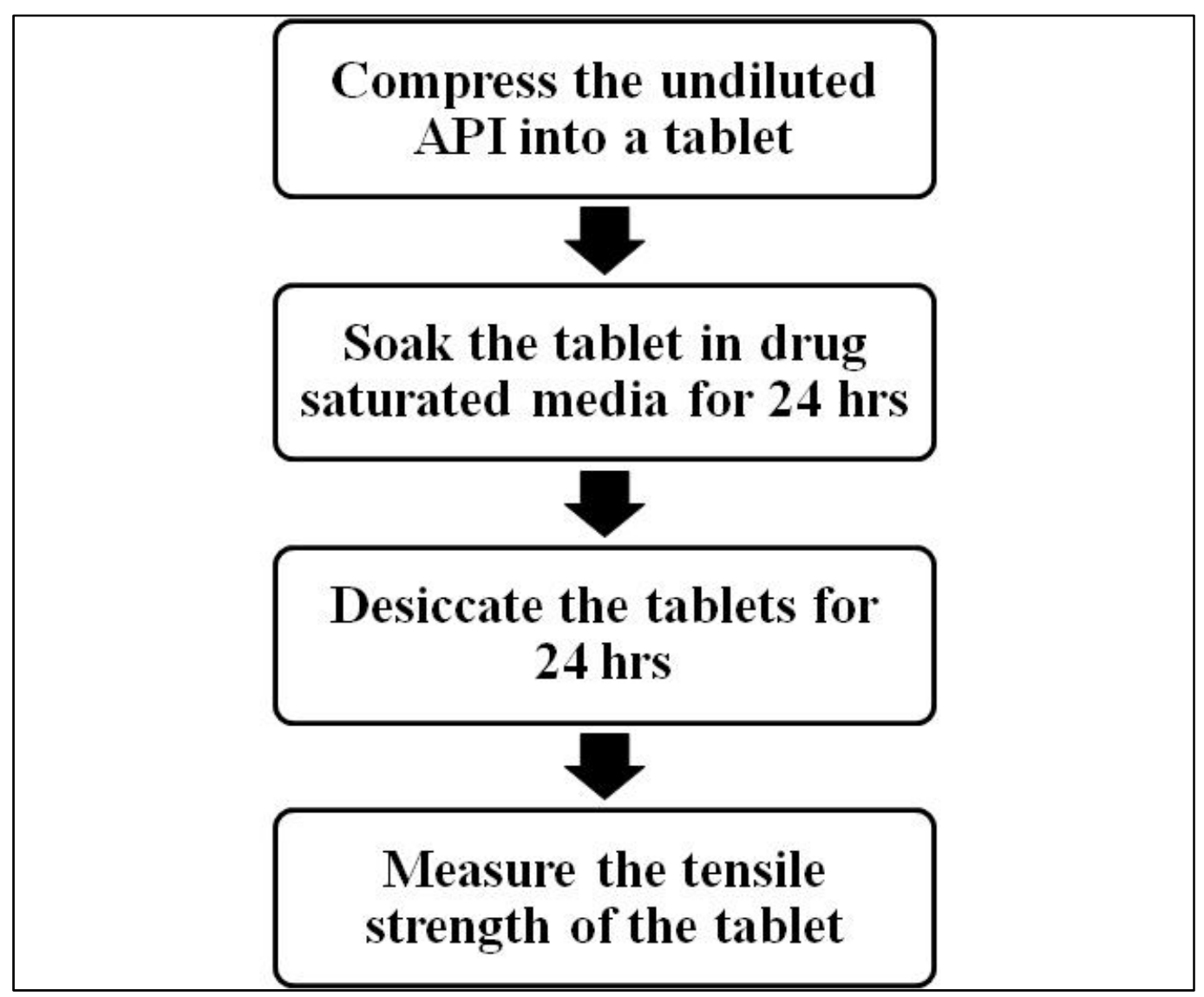

Figure 2-2. Scheme for API-API bonding study 


\subsubsection{Data analysis}

Mean particle size data for both the API's were used for comparison to evaluate the effect of compression force and effect of drug loading. Particle size data obtained after compression at various compression forces and drug loadings were subjected to a student $t$ test and $p$-values were presented.

\subsection{Results and Discussion}

\subsubsection{Particle size analysis and SEM characterization}

In order to introduce the general process of evaluation, Figure 2-3(a) to (d) present the particle size distribution of furosemide and griseofulvin before compression and after compression at $2 \mathrm{mT}$ at $30 \%$ drug loading. The particle a size of API's used in the studies was confirmed by measuring particle size by laser diffraction technique. The particle size distribution was represented in volume frequency percent. The mean particle size of furosemide and griseofulvin before compression was found to be $5.04 \pm 0.5$ and $13.96 \pm 0.23 \mu \mathrm{m}$ respectively. Size distribution curve for micronized furosemide showed a bi-modal distribution whereas for griseofulvin the particle size distribution found to be a unimodal distribution. Figure 2-4(a) to (d) shows the SEM images of micronized furosemide and griseofulvin before and after compression and disintegration.

Figure 2-3(b) and (d) shows the particle size distribution of furosemide and griseofulvin particles after compression. Micronized particles of furosemide formed large agglomerates after compression into tablet at $2 \mathrm{mT}$ at $30 \%$ drug loading which results in shifting of its distribution to right side skew distribution in Figure 2-3(b). The distribution of griseofulvin after compression was similar to initial particle size distribution but the size of the particles was varied. SEM images of furosemide and griseofulvin were also correlating with their respective distributions before and after compression. Surface morphology of furosemide particles after compression was changed to big large chunks due to formation of agglomerates during compression.

Furosemide API particles before compression shown in Figure 2-4(a) were crystalline in structure and like polygonal, cubical and rod in shape. According to de Villiers et al, the micronized crystal form influences the cohesive properties and that in turn affects its powder properties like flow, wettability, size distribution and density. ${ }^{100}$ SEM images of initial particles of griseofulvin in Figure 2-4(c) show crystals and pyramid and elongated rod like shapes. Figure 2-4(d) shows the change in crystals habit of griseofulvin (acicular and bipyramidal to aggregated particles) during compression at $2 \mathrm{mT}$ force. Compression of hydrophobic micronized API's along with other excipients at high compression force of $2 \mathrm{mT}$ at $30 \%$ drug loading results in the formation of strong agglomerates which thereby could alter the dissolution and disintegration process. 

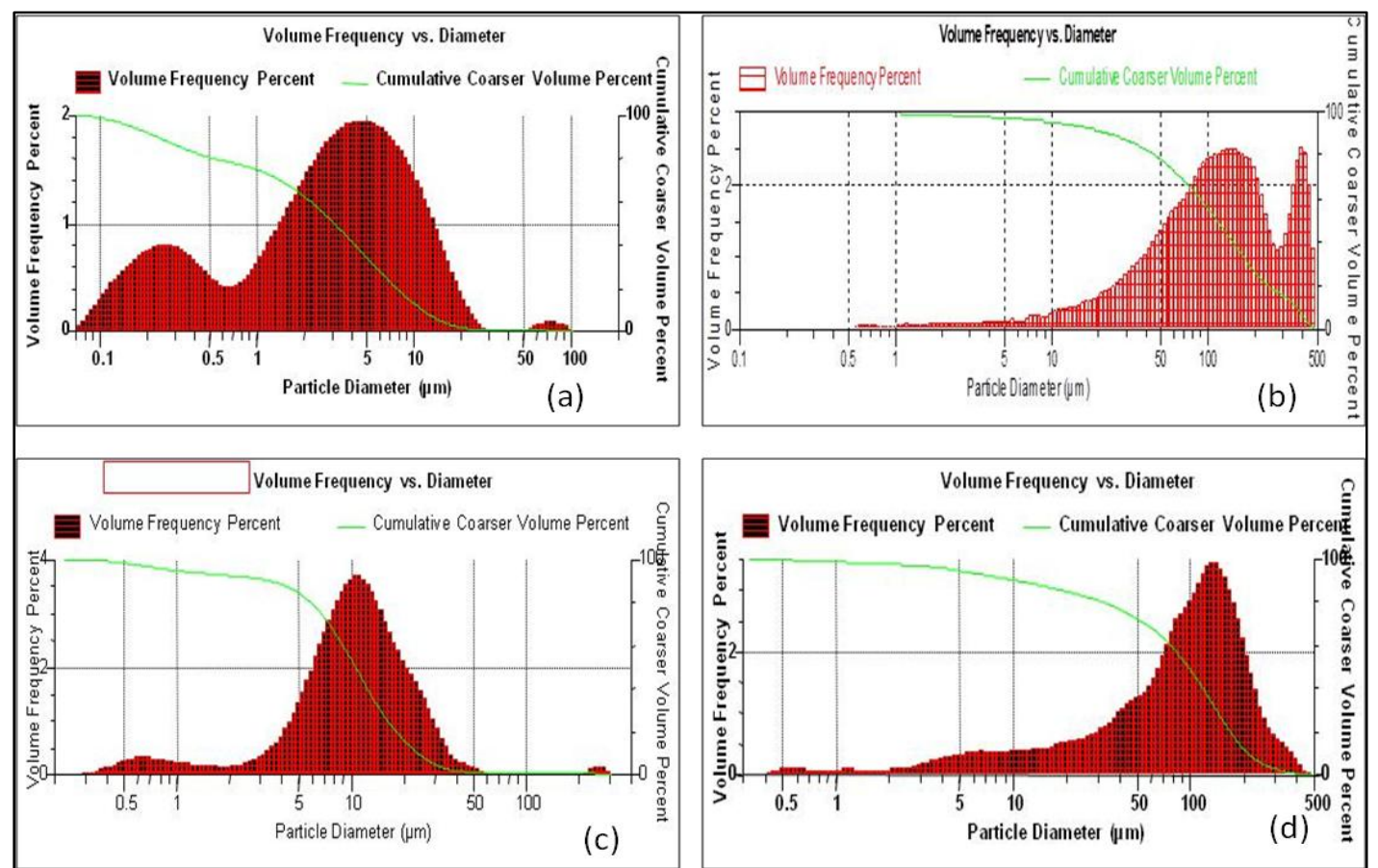

Figure 2-3. Particle size distribution of furosemide and griseofulvin API particles

a) Furosemide particles before compression

b) Furosemide particles after compression at $2 \mathrm{mT}$ with $30 \%$ drug loading

c) Griseofulvin particles before compression

d) Griseofulvin particles after compression at $2 \mathrm{mT}$ with $30 \%$ drug loading 


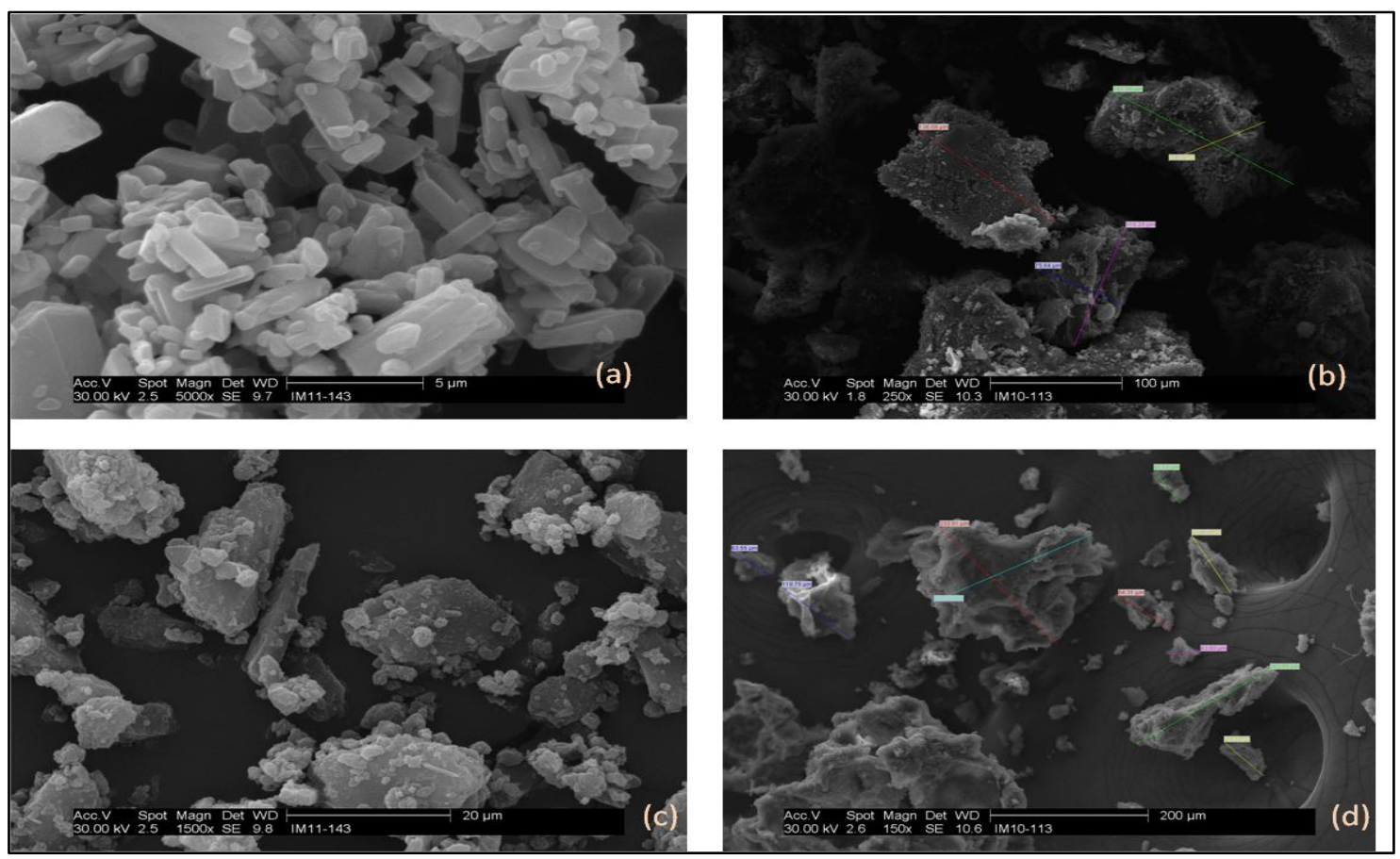

Figure 2-4. SEM images of furosemide and griseofulvin API particles

a) Pure furosemide particles before compression

b) Furosemide particles after compression at $2 \mathrm{mT}$ with $30 \%$ drug loading

c) Pure griseofulvin particles before compression

d) Griseofulvin particles after compression at $2 \mathrm{mT}$ with $30 \%$ drug loading 


\subsubsection{Validation of disintegration process and assay of filtered residue}

The filtered residue after dissolution of the soluble excipients was evaluated for furosemide and griseofulvin and was accepted as a viable sample if the assay was above 95\% API. This was considered as representative of the process and we further proceeded with that residue for characterization and particle size estimation. Validation results of disintegration process for furosemide and griseofulvin tablets compressed at $2 \mathrm{mT}$ with $30 \%$ drug loading is presented in the Table 2-4. The assay of furosemide after disintegration at time intervals of $0.5,1.0 \mathrm{hr}$ were $95.67,99.01$ respectively. As the disintegration time increases the soluble excipients inside the tablets undergoing complete dissolution results in the assay of $99.06 \%$ after $2 \mathrm{hrs}$. Similarly the griseofulvin tablet disintegration process was validated and the assay after disintegration at time intervals $0.5,1.0$ and $2.0 \mathrm{hrs}$ were $92.10,95.70$ and $99.73 \%$ respectively.

\subsubsection{Effect of compression force and drug loading}

Figures 2-5 and 2-6 represents the effect of compression force and drug loading on micronized furosemide and griseofulvin particles. An increase in the particle size was observed for both the API's with increase in compression force and increase in drug loading. There are several mechanisms responsible for increase in particle size of large agglomerates of drug during compression. The micronized furosemide particles with mean particle size of $5.04 \pm 0.5 \mu \mathrm{m}$ were increased to $14.16 \pm 3.6 \mu \mathrm{m}, 42.7 \pm 4.1 \mu \mathrm{m}$, $39.99 \pm 2.7 \mu \mathrm{m}$ and $65.79 \pm 2.8 \mu \mathrm{m}$ with $10 \%$ drug loading at $0.5,1.0,1.5$ and $2.0 \mathrm{mT}$ compression forces respectively. Similarly the micronized furosemide agglomerates into $12.29 \pm 8.5 \mu \mathrm{m}, 73.12 \pm 7.9 \mu \mathrm{m}, 84.24 \pm 2.3 \mu \mathrm{m}$ and $97.14 \pm 15.3 \mu \mathrm{m}$ with $20 \%$ drug loading at $0.5,1.0,1.5$ and $2.0 \mathrm{mT}$ compression forces respectively. With increasing drug loading to $30 \%$ the micronized furosemide particles formed large agglomerates of mean particles sizes $35.85 \pm 11.2 \mu \mathrm{m}, 139.5 \pm 6.8 \mu \mathrm{m}, 144.65 \pm 6.3$ and $150.1 \pm 17.8 \mu \mathrm{m}$ at $0.5,1.0,1.5$ and $2.0 \mathrm{mT}$ compression forces respectively. No significant difference was observed among compression forces in agglomeration of particles at all the furosemide drug levels ( $p>0.05$ ) whereas a significant difference was observed in particle size increase during compression compared for drug loadings at various compression forces $(\mathrm{p}<0.05)$. Compared to starting furosemide particle size the drug loading and compression force increased the particle size during compression.

The initial micronized griseofulvin particles with mean particle size of $13.96 \pm$ $0.23 \mu \mathrm{m}$ agglomerated to $30.36 \pm 1.06 \mu \mathrm{m}, 42.89 \pm 1.85 \mu \mathrm{m}, 49.39 \pm 0.88 \mu \mathrm{m}$ and 50.35 $\pm 1.15 \mu \mathrm{m}$ with $15 \%$ drug loading at $0.5,1.0,1.5$ and $2.0 \mathrm{mT}$ compression forces respectively. Likewise the micronized griseofulvin particles agglomerated together during compression with $30 \%$ drug loading into sizes $32.41 \pm 3.5 \mu \mathrm{m}, 55.00 \pm 7.2 \mu \mathrm{m}$, $93.37 \pm 6.4 \mu \mathrm{m}$ and $111.46 \pm 5.7 \mu \mathrm{m}$ at $0.5,1.0,1.5$ and $2.0 \mathrm{mT}$ compression forces respectively. Whereas at $60 \%$ drug loading the micronized griseofulvin formed larger agglomerates with mean particle sizes $51.02 \pm 8.1 \mu \mathrm{m}, 81.22 \pm 32.89 \mu \mathrm{m}, 108.9 \pm 5.1 \mu \mathrm{m}$ and $69.82 \pm 16.96 \mu \mathrm{m}$ at $0.5,1.0,1.5$ and $2.0 \mathrm{mT}$ compression forces respectively. 
Table 2-4. Validation of disintegration process

\begin{tabular}{ccc}
\hline Time (hr) & Furosemide (\%) & Griseofulvin (\%) \\
\hline 0.5 & 95.67 & 92.10 \\
1.0 & 99.01 & 95.70 \\
2.0 & 99.86 & 99.73 \\
\hline
\end{tabular}




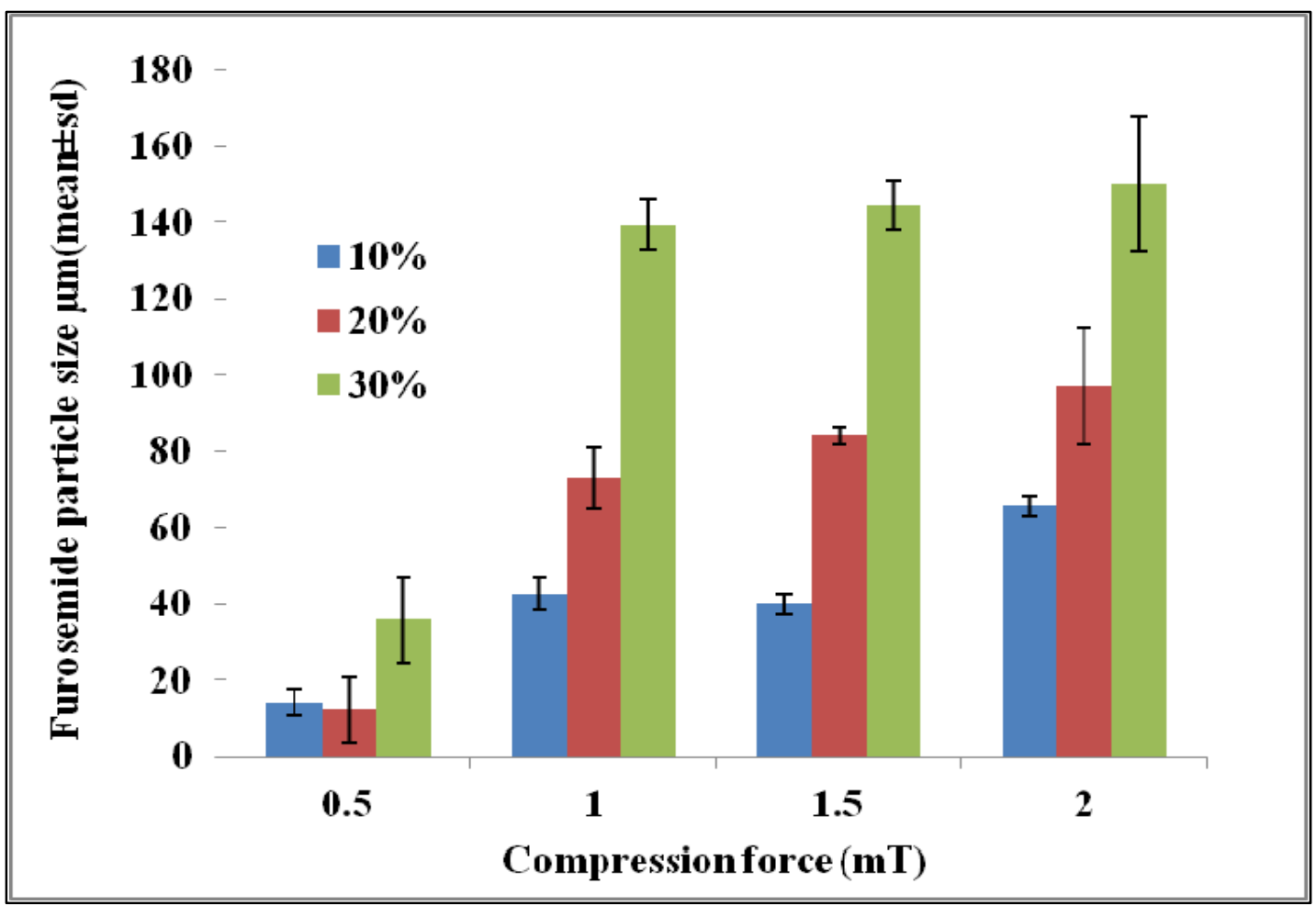

Figure 2-5. Effect of compression force and drug loading on furosemide particles 


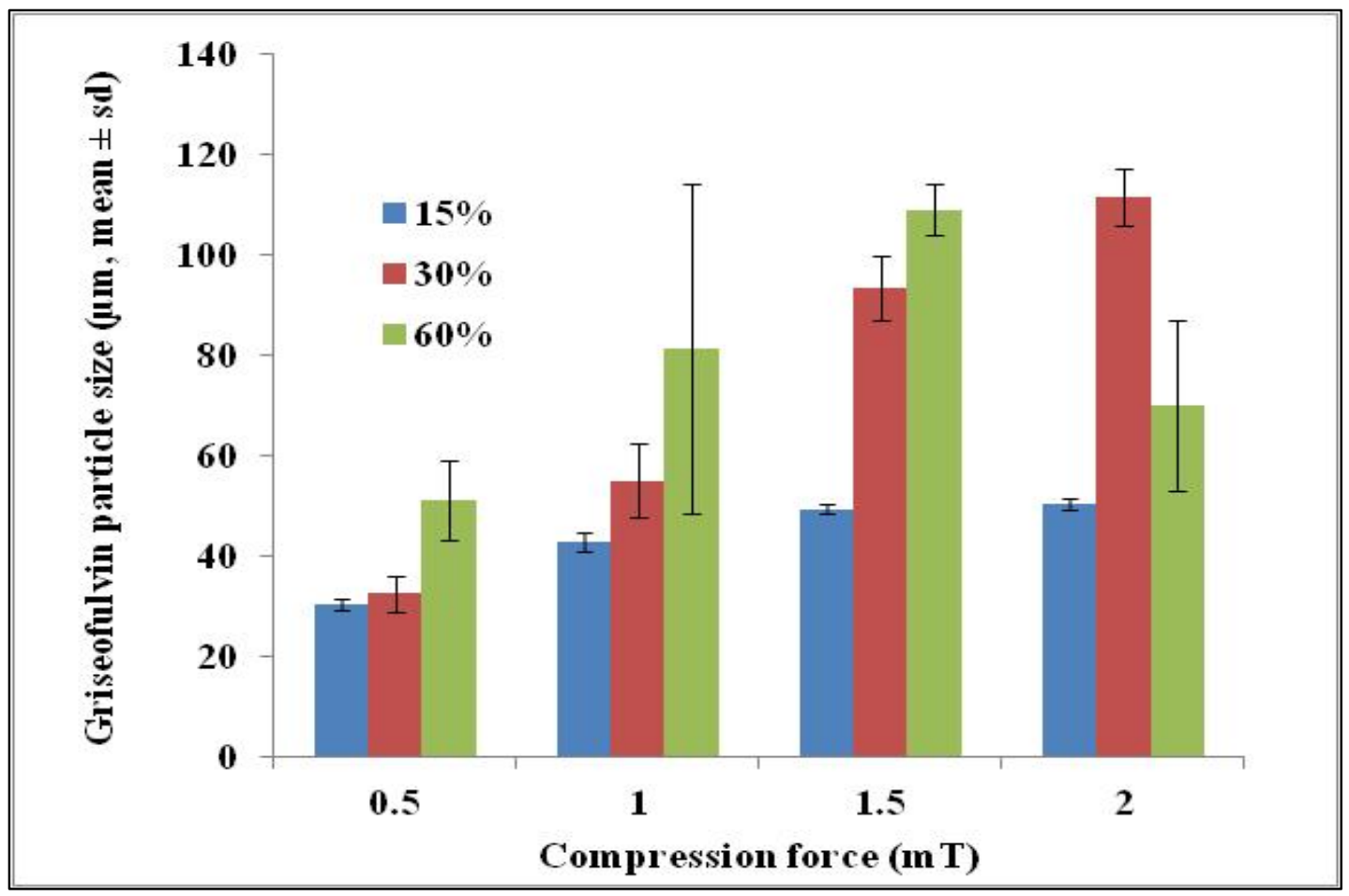

Figure 2-6. Effect of compression force and drug loading on griseofulvin particles 
Fracturing of the formed agglomerates at $2 \mathrm{mT}$ compression force might be one of the responsible mechanisms for nonlinearity of agglomerate formation with compression forces. Significant difference was observed among compression forces in agglomeration of particles at $15 \%$ and $30 \%$ of griseofulvin drug levels $(\mathrm{p}<0.05)$ whereas at $60 \%$ drug loading no significant difference was observed in particle size increase during compression compared for drug loadings at various compression forces ( $p>0.05)$. Compared to starting griseofulvin particle size the drug loading and compression force increased the particle size during compression. The increase in micronized griseofulvin particle size during compression may be attributed to solid bridge formation. The extent of particle agglomeration and the size of agglomeration during compression increases with increasing drug loading and compression force. The difference in the particle agglomeration during compression by increasing the drug content might be due to protection effect of lactose in lower drug content level and more interaction of drug-drug particle in high levels of drug content. ${ }^{101}$ And also high surface energy and greater surface area of micronized powders could also be a contributing factor in the formation of agglomerates during compression.

\subsubsection{Modulated differential scanning calorimetry}

Figures 2-7 and 2-8 represents thermograms of furosemide and griseofulvin API particles and after compression respectively. Figure 2-7 represents the sharp endothermic peak at $208^{\circ} \mathrm{C}$ in reverse heat flow for furosemide before compression sample represents the crystalline nature of it. After compression at $2 \mathrm{mT}$ with $30 \%$ drug loading the furosemide did not change its solid state nature at high compression forces and showed nearly similar endothermic peak at $210^{\circ} \mathrm{C}$. In Figure 2-8, griseofulvin particles showed similar endothermic peaks at $219^{\circ} \mathrm{C}$ before and after compression at $2 \mathrm{mT}$ with $30 \%$ drug loading. This melting endotherm corresponds to the crystalline nature of griseofulvin. Similarly griseofulvin did not undergo any polymorphic conversion at high compression forces studied at $2 \mathrm{mT}$ compression force.

\subsubsection{Powder X-ray diffraction studies}

Figures 2-9 and 2-10 represents the difractograms of furosemide and griseofulvin API particles before and after compression respectively. In Figure 2-9 micronized furosemide before compression showed intense peaks at 5.89, 12.05, 18.01 and 24.65 degrees. This confirms the crystalline nature of the furosemide. After compression at $2 \mathrm{mT}$ for $30 \%$ drug loaded batch showed overlapping peaks for furosemide. This confirmed that there was no effect of compression force on polymorphic transformation of furosemide but some peaks at 5.89, 12.05 and 14.15 degrees were suppressed.

This suppression might be due to change in the crystal habit during the compression. In Figure 2-10 similar pattern of intense peaks were observed at 10.74, $13.18,14.78,16.47,23.89$ and 26.71 degrees for micronized griseofulvin particles before 


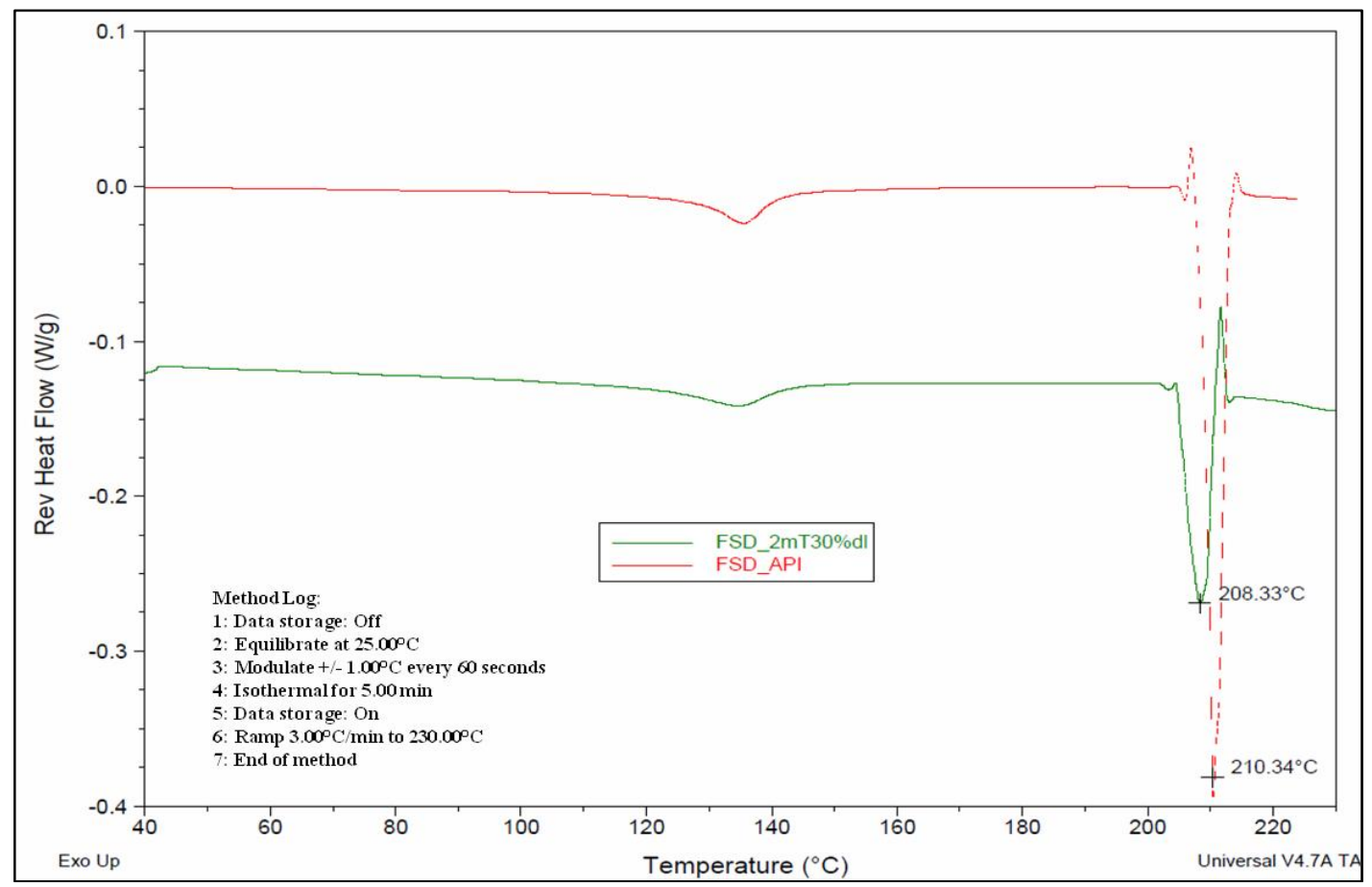

Figure 2-7. MDSC thermograms of furosemide before and after compression 


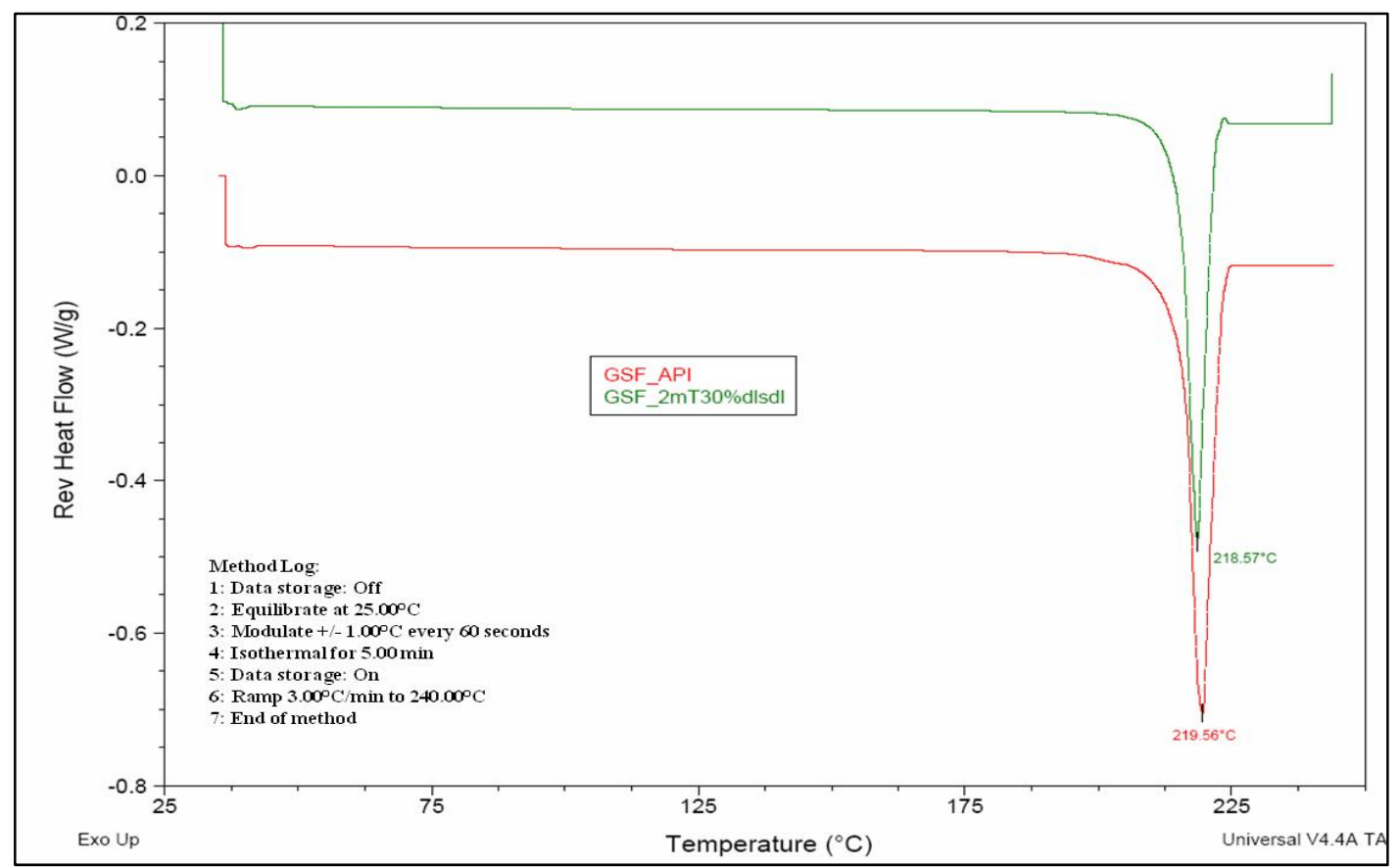

Figure 2-8. MDSC thermograms of griseofulvin before and after compression 


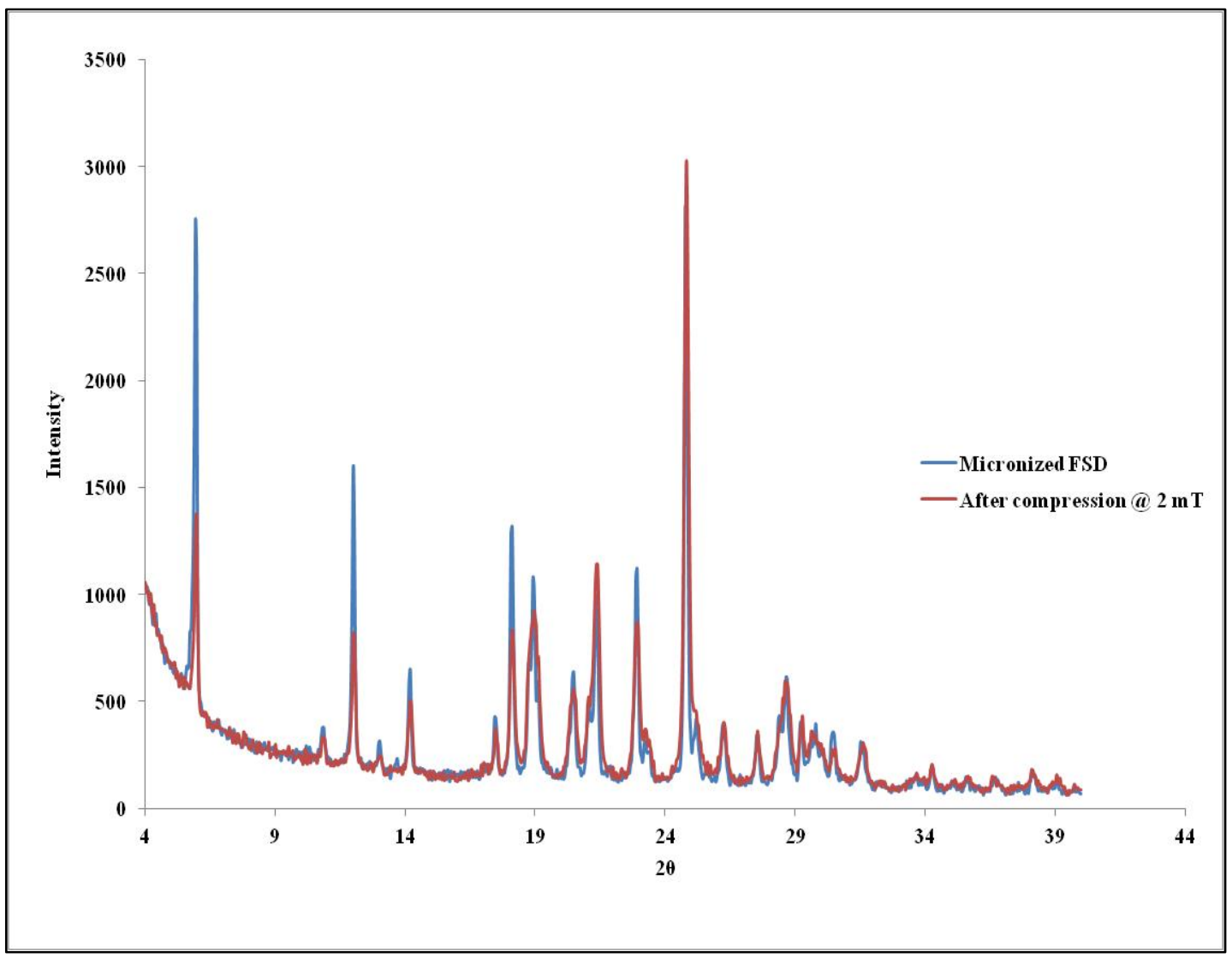

Figure 2-9. X-ray powder diffraction of furosemide particles 


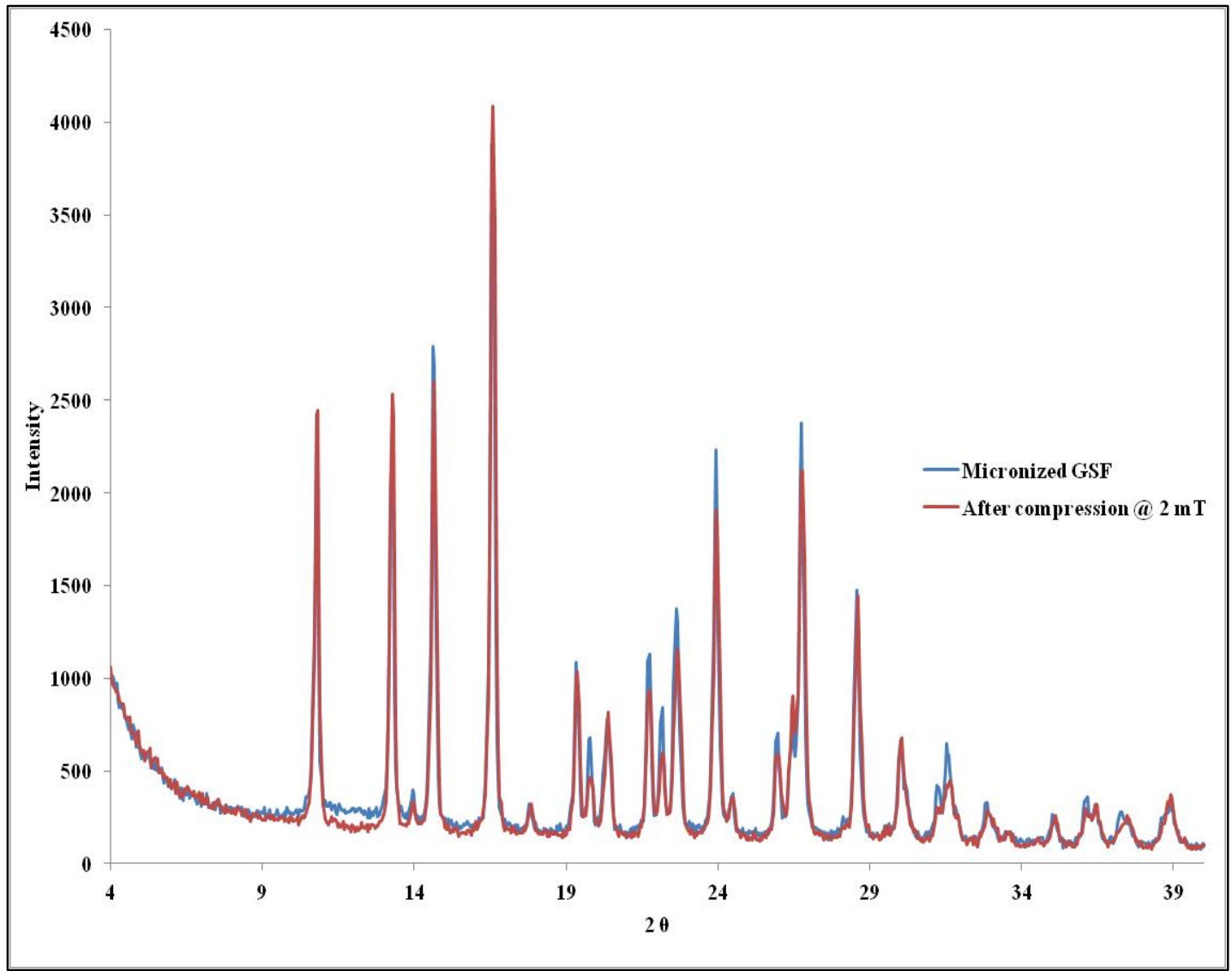

Figure 2-10. X-ray powder diffraction of griseofulvin particles 
and after compression with very weak suppression of peak intensities were observed after compression. This demonstrates the speculation of no polymorphic transformation during compression at $2 \mathrm{mT}$ compression force with $30 \%$ drug loading and also maintained the crystallinity of both the API's.

\subsubsection{Effect of diluent particle size}

Surface area and particle size distribution of two fractions of diluents, spray dried lactose and DiTab ${ }^{\circledR}$ were presented in Table 2-5. Gas adsorption technique results showed for the small size fractions of diluents showed larger specific surface area and larger size fractions showed small specific surface area. Effect of these size fractions on agglomeration of furosemide particles at $2 \mathrm{mT}$ compression force with $30 \%$ drug loading is presented in Figure 2-11. Blend of fraction- 2 with larger particle size and smaller specific surface area showed increase in size of furosemide particles during compression. This increase in drug particle size might be due to smaller surface area available for deposition of micronized drug on the surface of diluents. However, the diluents blend of fraction-1 with smaller particle size with larger specific surface area significantly $(\mathrm{p}<0.05)$ reduced the agglomeration of drug particles during compression and this might be due to availability of more contact area which prevention of drug-drug particle binding.

\subsubsection{Dissolution studies}

Generally dissolution rate also depends on the polymorphic forms of the drugs. High energies are needed in transition of the molecule into solution in the crystalline forms compared to transition of molecules in amorphous forms. Hence, the amorphous forms dissolve faster. However, dissolution of crystalline drugs may also change conversely due to imperfections generated during compression. So the energy required for transition into solution from these crystals may decrease and results in the increase in dissolution rate. ${ }^{102-104}$ Change in the dissolution rate distinctly can be observed at higher compression forces. The dissolution of particles after compression was studied rather tablets in this study to avoid any effect of compression force. Dissolution studies of furosemide and griseofulvin particles are presented in the Figures 2-12 and 2-13 respectively. The dissolution study of unprocessed bulk furosemide particles was not successful due its hydrophobic nature hence hinders the wetting of the particles.

Furosemide particle sizes 16.16 and $81.34 \mu \mathrm{m}$ were selected as a representative particle size for powder dissolution study from diluents (DiTab) size effect study. The agglomerates of furosemide formed during compression at $2 \mathrm{mT}$ with $30 \%$ drug loading were selected to evaluate particle size on dissolution rate instead tablets dissolution because the surface of these particles will be directly available to dissolution media. The rate of dissolution and the amount dissolved during study was slow and small for larger particles compared to smaller particles of furosemide. Around $56 \%$ of smaller size 
Table 2-5. Powder properties of diluents fractions

\begin{tabular}{cccc}
\hline Diluent & Physical property & Fraction-1 & Fraction-2 \\
\hline $\begin{array}{c}\text { Calcium phosphate di } \\
\text { hydrate } \\
(\text { DiTab) }\end{array}$ & $\begin{array}{c}\text { Particle size } \\
(\mu \mathrm{m})\end{array}$ & $37.90 \pm 0.31^{\mathrm{a}}$ & $215.01 \pm 0.24^{\mathrm{a}}$ \\
& $\begin{array}{c}\text { Surface area } \\
\left(\mathrm{m}^{2} / \mathrm{gm}\right)\end{array}$ & 1.0318 & 0.5889 \\
Spray dried lactose & $\begin{array}{c}\text { Particle size }(\mu \mathrm{m}) \\
\text { Surface area } \\
\left(\mathrm{m}^{2} / \mathrm{gm}\right)\end{array}$ & $92.36 \pm 0.59^{\mathrm{a}}$ & $233.30 \pm 19.45^{\mathrm{a}}$ \\
& 1.43 & 0.0931 \\
\hline
\end{tabular}

$\mathrm{a}-($ mean $\pm \mathrm{sd}, \mathrm{n}=3)$ 


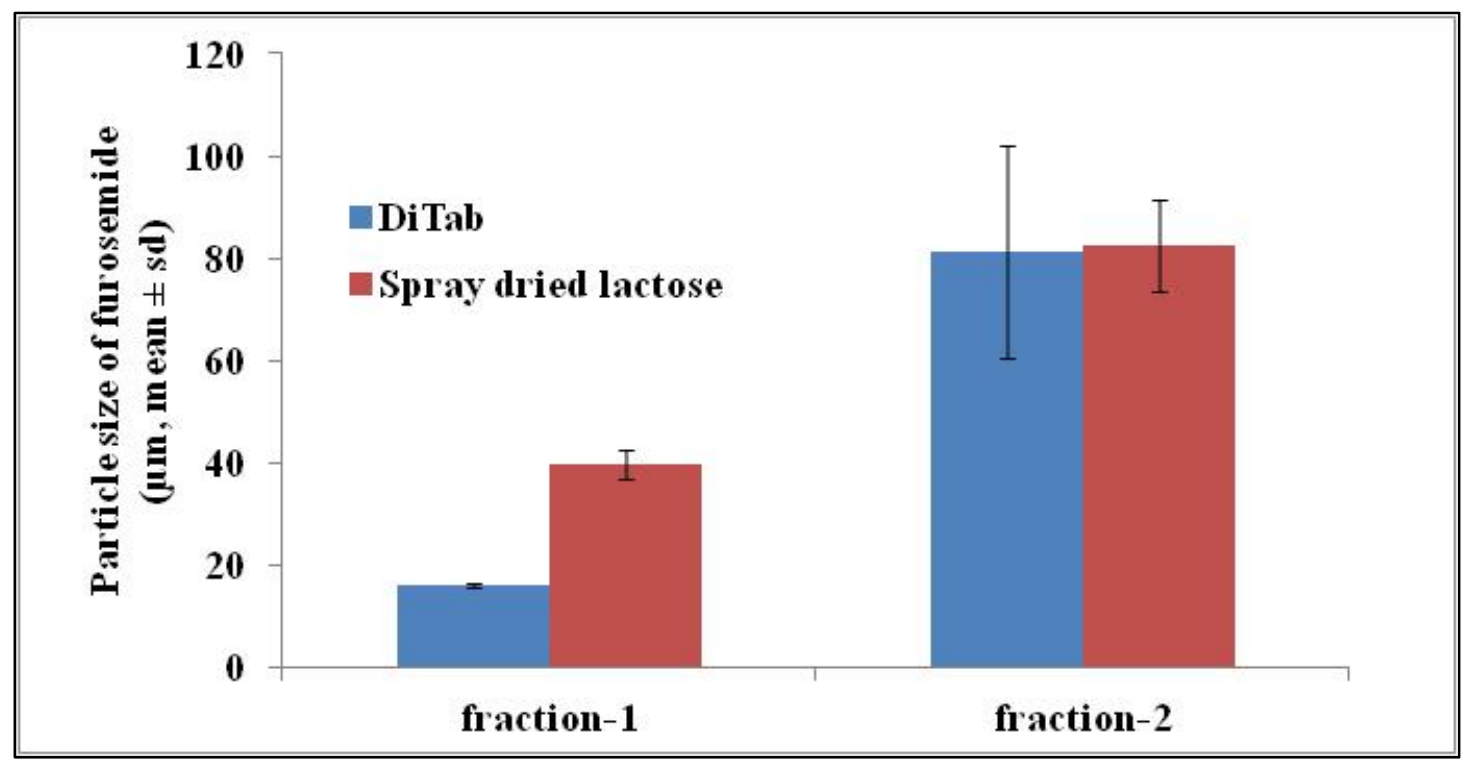

Figure 2-11. Effect of particle size of diluents on furosemide during compression at $2 \mathrm{mT}$ with $30 \%$ drug loading 


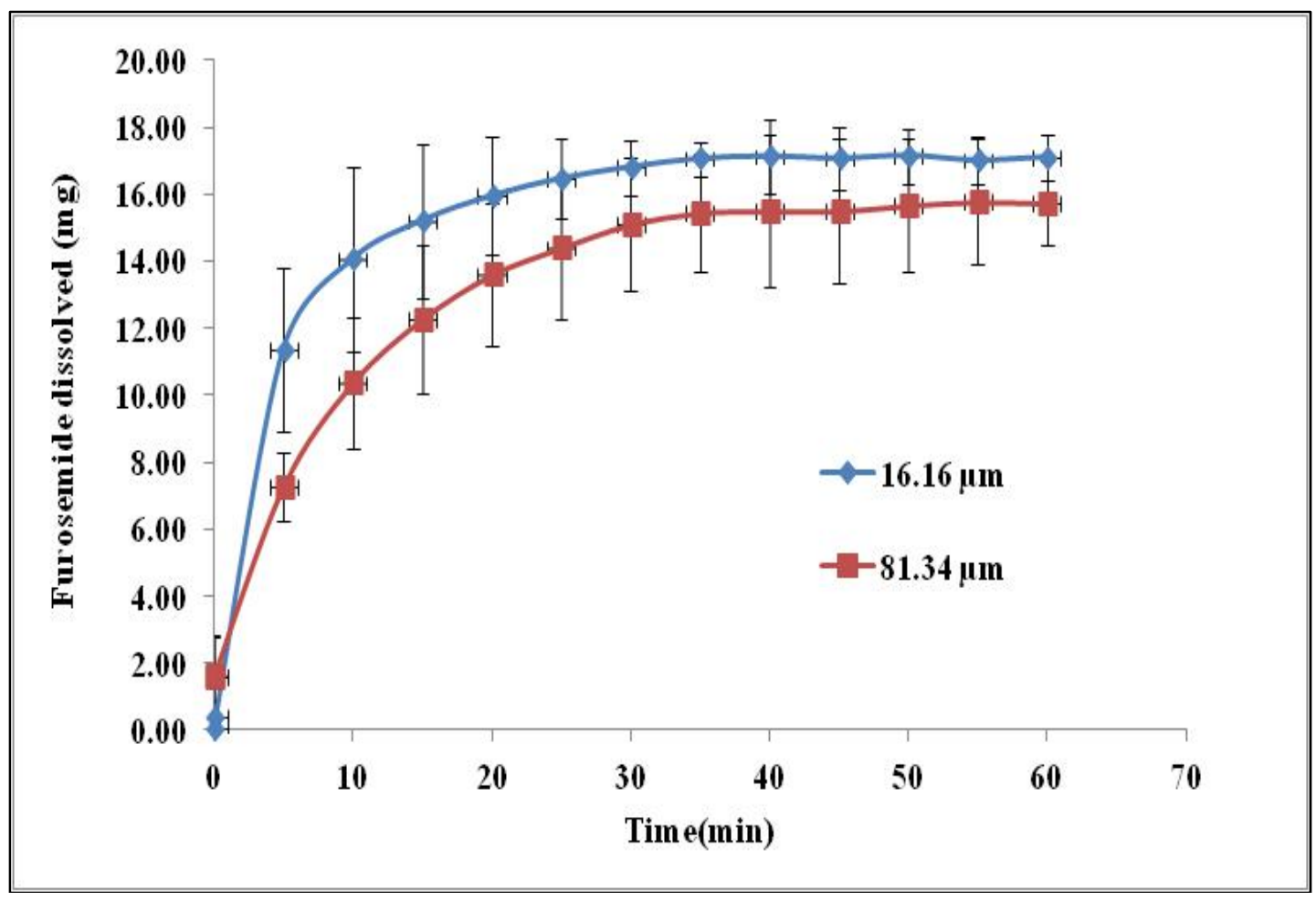

Figure 2-12. Dissolution of furosemide particles 


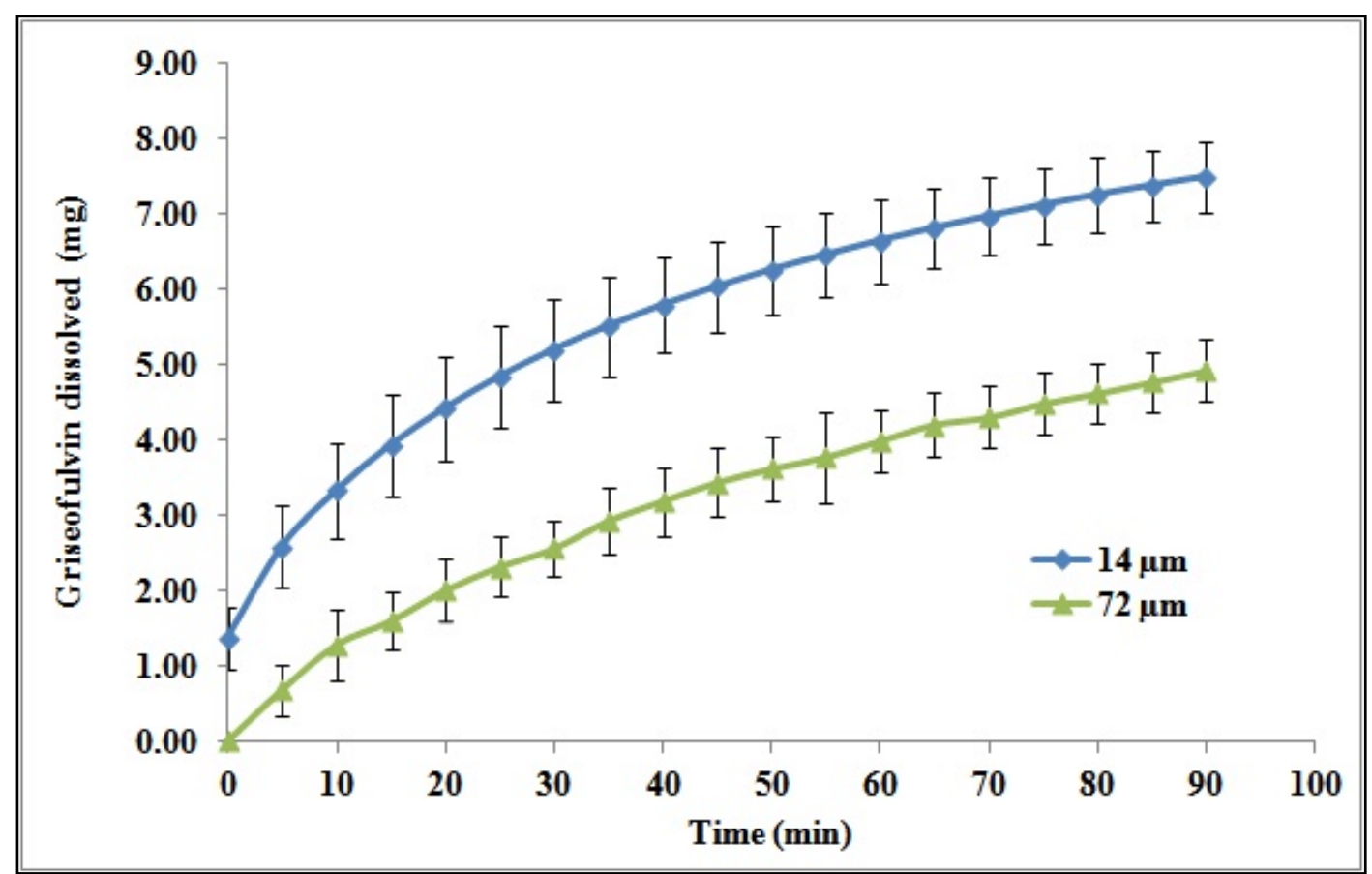

Figure 2-13. Dissolution of griseofulvin particles 
particles were dissolved within 5 min whereas only 36\% was dissolved for larger size agglomerates. Similar results were observed for griseofulvin powder particles.

Irrespective of the presence of wetting agent in the dissolution media and processing of the different size powders of griseofulvin agglomerates showed difference in dissolution. Approximately $12 \%$ of micronized griseofulvin particles got dissolved within 5 min but it took $30 \mathrm{~min}$ to dissolve same amount of large particles of griseofulvin. This decrease in dissolution clearly evident that hydrophobic micronized drug particles agglomerates during compression which altered the dissolution rate instead of enhancing.

\subsubsection{Drug-drug bonding mechanism}

For evaluating the bonding mechanism in drug-drug particle agglomeration micronized griseofulvin was selected due to its better compressible nature compared to furosemide. To avoid dissolution of griseofulvin during soaking of the compacts the media was pre saturated with it and its concentrations were presented in the Table 2-6. All the tablets were soaked in this media and tensile strength was measured after drying. Traditionally, based on the literature, assessment of tensile strength reveals what type of bonding occurs during compression. ${ }^{50}$ Moderate increase in dielectric constant not likely affect the solid bridge formation during compression, since solid bridges consists of continuous phase of solid material. The limitation of this test is solubility of the material at dielectric constants at greater extent which impacts the bonding structure of compacts. According to Figure 2-14 the tensile strength of all the tablets at increased compressed force were decreased initially and reached to plateau by increasing the dielectric constant of the liquid media. This plateau might be due to inability of dissipate agglomerated particles formed by solid bridge formation between the drug particles. Measurement of tensile strength will give a better estimate for evaluating the solid bridge formation in the compact. The tensile strength values at different dielectric constant media for griseofulvin at various compression forces were not significantly different $(\mathrm{p}>0.05)$ except at $1 \mathrm{mT}$ compression force $(\mathrm{p}<0.05)$. The accurate measurement of solid bridge formation between particles in the compact can be evaluated by calculating percent remaining tensile strength of the compact after testing the crushing strength of the soaked and dried tablets. Percent remaining tensile strength of the compact can be calculated by using Equation 2-2.

$$
R T s=\frac{T s_{(l)}}{T s_{(a)}} \times 100
$$

Where Ts ${ }_{(1)}$ and $\mathrm{Ts}_{\text {(a) }}$ are tensile strength of the compacts soaked in liquid and air respectively. Figure 2-15 represents the percent remaining tensile strength vs. dielectric constant. More than $60 \%$ remaining tensile strength was observed and this substantiates the formation of solid bridge during compression of griseofulvin alone at different compression force. 
Table 2-6. Solubility of griseofulvin in organic solvents

\begin{tabular}{ccc}
\hline Media & Dielectric constant & Solubility $(\mathbf{m g} / \mathbf{m L})$ \\
\hline Air/ vacuum & 1.00 & NA \\
Hexane & 1.89 & 0.010 \\
n-Butanol & 18.00 & 2.535 \\
Ethanol & 24.30 & 4.403 \\
\hline
\end{tabular}




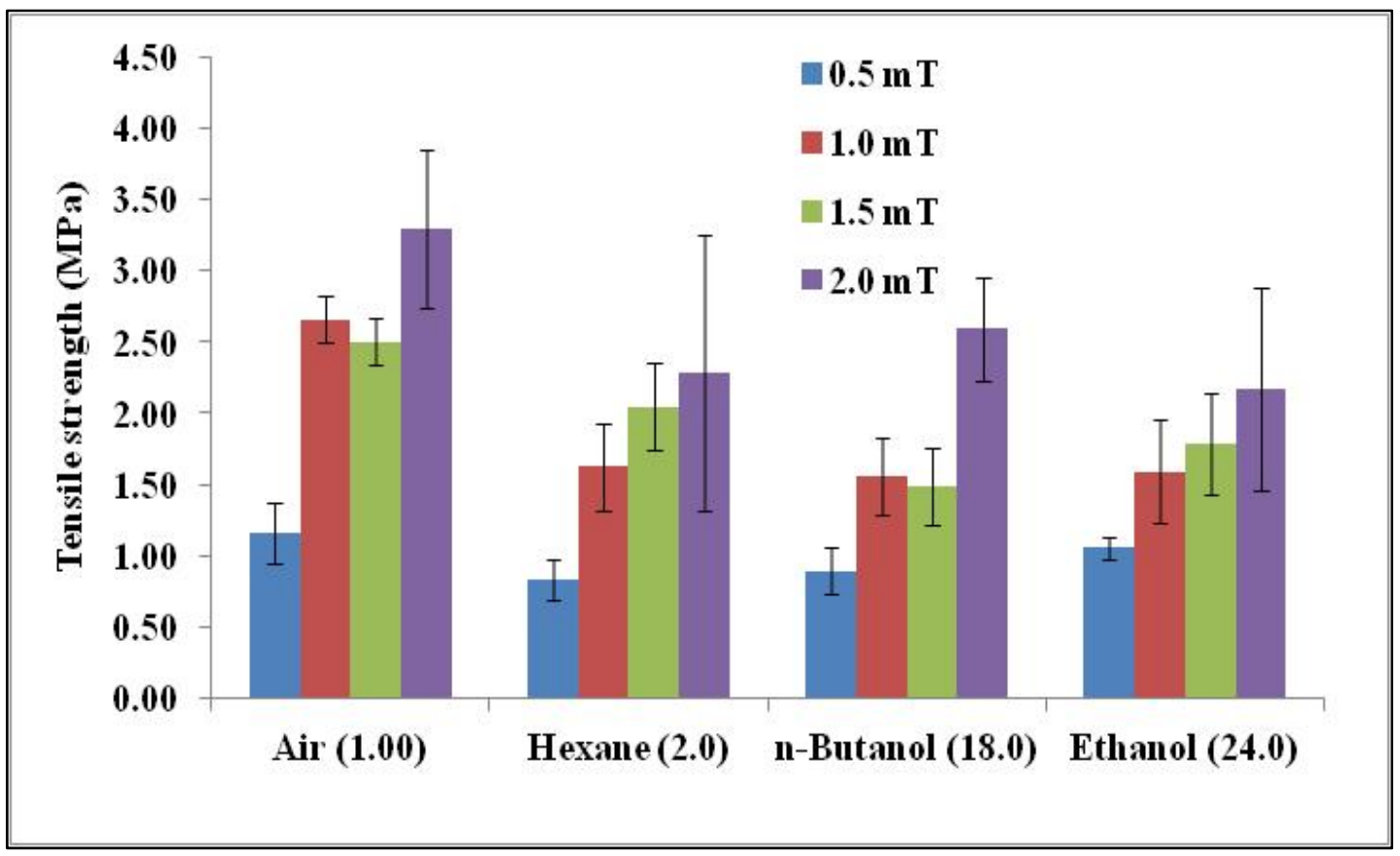

Figure 2-14. Effect of dielectric constant and tensile strength 


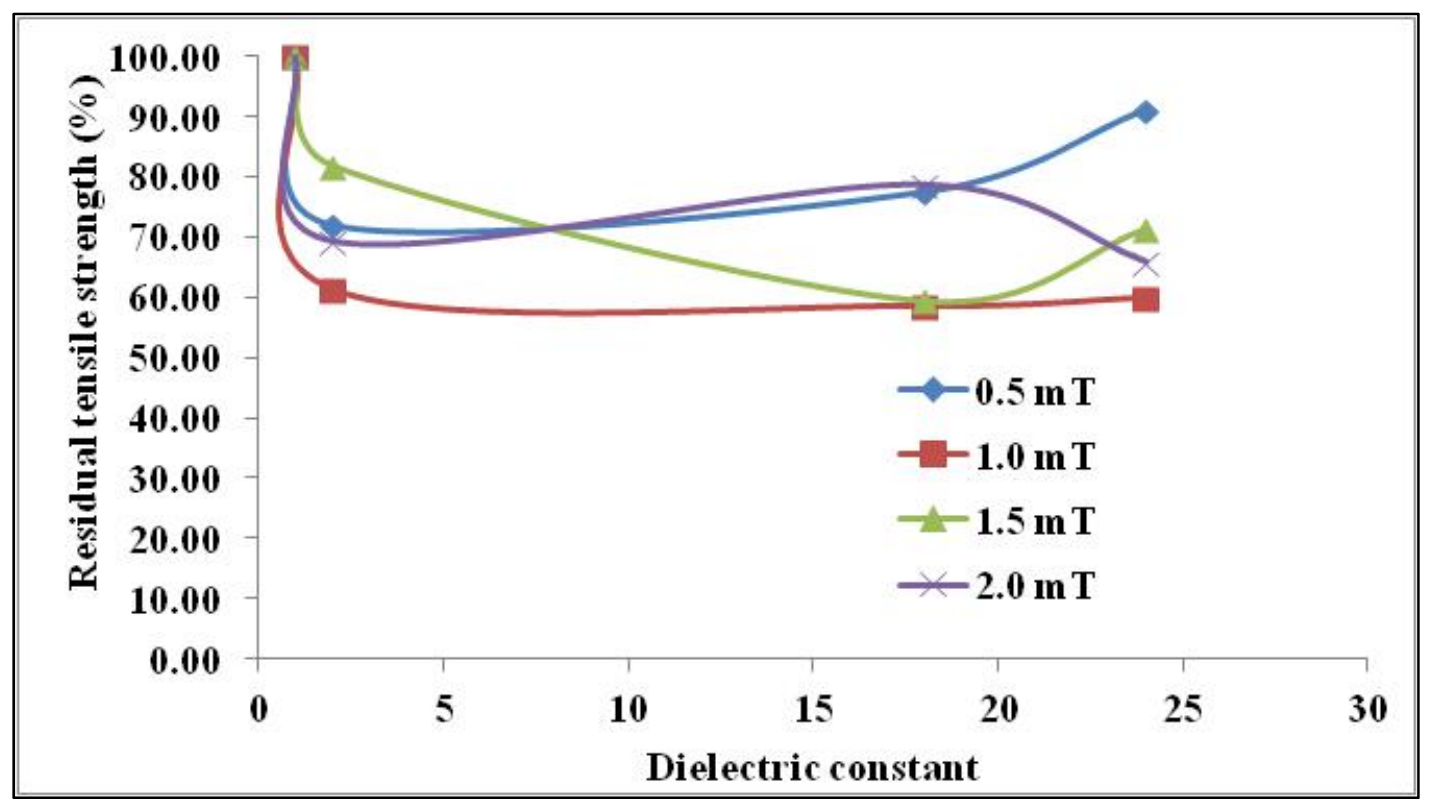

Figure 2-15. Effect of dielectric constant on remaining tensile strength of compacts 
The percent remaining tensile strength of compacts was not proportional to with the compression force. The remaining tensile strength of griseofulvin was significantly higher at the plateau level which suggests the greater tendency for griseofulvin to form solid bridges. Eriksson et al, described the variation in the predicted and actual tensile strength of the compacts might be due to fracture mechanic effect or a high degree of deformity of particles during compression. ${ }^{105}$ Based on these results more energy is required to break the formed agglomerates during compression of micronized griseofulvin particles. The increase in tensile strength due to fragmentation and formation of new surfaces can be ruled out since the starting particles itself are micron size.

\subsection{Conclusions}

In conclusion, the effect of compression force study on agglomeration of micronized furosemide during compression showed significant increase in its particle size compared to initial particle size but that increase was not significant among the (consistent as compression force increased) compression forces. This consistent increase after $1 \mathrm{mT}$ compression force might be large enough to exceed the elastic limit of furosemide particles that lead to breakage of furosemide aggregates by fracturing. Increasing drug loading study for furosemide showed significant increase in furosemide particle size during compression. 3 to 16 times increase from initial furosemide particles in the effect of diluent particle size study on agglomeration of furosemide particles during compression can be concluded that diluents with larger surface area $\left(1.03 \mathrm{~m}^{2} / \mathrm{gm}\right.$ or smaller particle size $(37.90 \mu \mathrm{m})$ are more suitable to avoid API agglomeration during compression compared to diluents with larger size and smaller surface area. This might be due to reduced adhesion of micronized API particles on large size/smaller surface area diluents and higher deposition of API particles on smaller size/large surface area diluents which provide more interparticulate contact area. Increasing the compression force and drug loading study for griseofulvin particle agglomeration significantly increased the particle size of griseofulvin during compression except at high drug loadings. The results of the characterization studies SEM, thermal studies and powder x-ray diffraction for furosemide and griseofulvin before and after compression concluded that high compression forces modify the surface properties but had no effect on polymorphic transformation during compression. The powder dissolution results for both the API's at different particles size showed effect of surface area exposed to dissolution media. The dominating bonding mechanism responsible for agglomerate formation was found to be solid bridge formation between API particles during compression. 


\section{CHAPTER 3. PREVENTION OF DRUG-DRUG PARTICLE AGGLOMERATION DURING COMPRESSION}

\subsection{Introduction}

During the tablet compression the formula composition and the arrangement of the various particles and their nearest neighbor contacts in the die influence the bonding which occurs. Micronized or nano sized particles of hydrophobic drugs can agglomerate into larger particles due to their inter-particular bonding of various types between these particles during tablet compression. Agglomeration of these drug particles can be prevented by treating these particles with particle agglomeration inhibitors (PAI) and/or by using various processing techniques like roller compaction and spray drying before compression.

The desirable characteristics of PAI's such as nonionic block copolymer, waxes, low melting polyethylene glycols and these should not interact chemically with the active pharmaceutical ingredients. PAI's should possess good wetting properties, soft (low melting point), large surface area (small in size), hydrophilic (high HLB) in nature and should possess excellent compressibility. Various methods such as film coating of crystals, dispersion coating with soluble film former can reduce generation of hot spots which affects adhesion during compression. ${ }^{106} \mathrm{~A}$ common process in pharmaceutical industry for manufacturing of tablets is powder mixing. Powder mixtures are classified into two major groups, free-flowing mixtures, and mixtures containing interactive constituents. 107

Chiou et al. found a marked increase in dissolution rate for griseofulvin with PEG dispersions. The physical nature of these systems was not well characterized. This marked increase in dissolution was due to the decreased particle size in this molecularly or colloidally dispersed griseofulvin in matrices of polyethylene glycol polymers. ${ }^{108}$

Gonnissen et al. described the change in the physical properties of various drugs, particularly surface properties by co-processing with excipients together using spray drying technique. This platform technology can be useful in developing ready-tocompress powder mixtures to improve poor compactability of drugs. ${ }^{109,110}$ According to Makai et al. spray drying with alginates and lactose showed marked increase in the polarity of the surface of the particles. ${ }^{111}$ Granules containing too high concentration of super disintegrant can slow down the drug release from tablets due to viscous barrier surrounding the particles. ${ }^{112}$

Expensive and time consuming process such as co-micronization of fenofibrate a poor bioavailable antihyperlipoproteinemic agent improved the bioavailability significantly greater extent with solid surfactant (sodium luaryl sulfate) than that simply adding to formulation. Adsorption of surfactants on the surface of the solid is very important for various applications. 
Recently roller compaction, a dry granulation process has been commonly used for improving the dissolution rate of hydrophobic drugs. Mitchell et al. describes the roller compaction with HPMC for poorly soluble drugs enhanced the dissolution rate due to its micro environmental surfactant mechanism. ${ }^{113}$

Two hydrophobic micronized furosemide and griseofulvin were selected as model drugs. Micronized furosemide and griseofulvin were individually compressed into tablets along with surface active gents having large surface area and good wetting and compressible properties. Today nanotechnology is increasing interest. Development of tablet formulation with nano sized particles is a challenging task due to aggregation of these particles during compression. Thus, very few tablet products with low dose are currently available in the market based on the nanocrystal technology.

The objective of this study was to study the effect of various surface active agents and various processing methods like physical mixing, roller compaction and spray drying in prevention of drug-drug particle agglomeration during compression. Effect of compression force also evaluated after treating the API with above mentioned methods and particle agglomeration inhibitors (PAI). Particle sizes of these drugs were measured before and after compression as the primary distinguishing evaluation parameter in this work.

\subsection{Materials and Methods}

\subsubsection{Materials}

Furosemide (micronized API) was purchased from Teva Pharmaceuticals, Israel. Micronized griseofulvin was a generous gift from Wuhan Yuancheng Technology, China. Modified spray dried lactose 315 (Foremost ${ }^{\circledR}$ NF Lactose) was purchased from Foremost Forms, Baraboo, WI. Mannitol SD 200, hydroxy propyl starch (Lycoat RS 720) were gift samples from Roquette America Inc, Geneva, IL. Croscarmellose sodium (Ac-Di-Sol ${ }^{\circledR}$ ) was obtained from FMC biopolymer, Philadelphia. Poloxamer 188 (Lutrol ${ }^{\circledR}$ micro 68) was obtained as a gift sample from BASF Corporation, New Jersey. Polyethylene glycol (PEG 3350) was obtained from Dow chemicals, USA. Sodium benzoate was purchased from Spectrum Chemicals Manufacturing Corporation, California. Magnesium stearate was purchased from Mallinckrodt, New Jersey.

\subsubsection{Methods}

\subsubsection{Effect of physical mixing}

Initially API's and PAI's were mixed together geometrically and passed through \# 60 stainless steel mesh along with spray dried lactose monohydrate and mixed together 
by geometric mixing by passing through \#60 stainless steel mesh twice and finally AcDi-Sol was blended in a plastic bag for $5 \mathrm{~min}$. All the materials were accurately weighed according to formulation in Tables 3-1 to 3-5.

\subsubsection{Effect of roller compaction}

The blend of API and PAI was prepared to prevent API-API agglomeration during compression by roller compaction. To increases the API-PAI contact during processing and to avoid segregation the API was blended with PAI which has larger surface area and smaller in particle size than the unmodified PAI. To obtain smaller particles of PEG 3350 the bulk PEG 3350 was sifted through mesh \# 100 and a fraction of particle size range less than $149 \mu \mathrm{m}$ was collected. For micronized Lutrol F68 the supplier provided the average particle size of $50 \mu \mathrm{m}$. Similarly spray dried lactose from bulk was selected by sieving through a stack containing mesh \# 80 and mesh \#200. The fraction that passed through mesh \#80 and retained on mesh \#200 was selected for this study. For preparation of blend for roller compaction initially the micronized griseofulvin/furosemide and PEG 3350/ Lutrol F68 micro were blended by passing through mesh \# $140(105 \mu \mathrm{m})$ based on drug to particle agglomeration inhibitor ratios mentioned in the Table 3-4 and Table 3-5.

The pre-blend was made into ribbons using TFC-Labo (Vector Corporation) mini roller compactor. Compaction pressure was adjusted to $5 \mathrm{MPa}$ and roll speed was kept at $2 \mathrm{rpm}$ and $20 \mathrm{rpm}$ feed rate was adjusted to feed blend. Thick and uniform ribbons were produced with above parameters and the ribbons were passed through a \# 40 mesh screen and blended with spray dried lactose and Ac-Di-Sol for 15 minutes before compression.

\subsubsection{Effect of spray drying}

A suspension of micronized furosemide was prepared by weighing 2 parts of Mannitol and 1 part of micronized furosemide in $100 \mathrm{~mL}$ of distilled water and stirred for $4 \mathrm{hrs}$ to obtain a homogeneous suspension. The suspension was spray dried using a BUCHI-290 (Switzerland) spray dryer with $150^{\circ} \mathrm{C}$ inlet and $95^{\circ} \mathrm{C}$ outlet temperature; the aspirator capacity was $100 \%$, the feed rate was $20 \%$ and $60 \mathrm{ml} / \mathrm{hr}$ of airflow. Mannitol and furosemide co-processed powder was collected in cyclone separator. For comparison another batch of physical blend was prepared according to the formulation given in Table 3-6 and compressed into tablets at various compression forces. The co-processed powder was blended with remaining excipients present in Table 3-7 and compressed into tablets. Preparation procedure for co-processed powder is given in Figure 3-1. Similarly a furosemide suspension was prepared by weighing 5 parts of micronized furosemide and 1 part of hydroxyl propyl starch (Lycoat RS 720) in $100 \mathrm{ml}$ of distilled water and stirred for $4 \mathrm{hrs}$ to get homogenous suspension. 
Table 3-1. Formulations for effect of physical mixing on griseofulvin particles

\begin{tabular}{ccccccc}
\hline Ingredients & F-1 & F-2 & F-3 & F-4 & F-5 & F-6 \\
\hline Griseofulvin (\%) & 30.00 & 30.00 & 30.00 & 30.00 & 30.00 & 30.00 \\
PEG 3350 (\%) & 15.00 & 30.00 & 60.00 & - & - & - \\
$\begin{array}{c}\text { Lutrol F68 micro } \\
(\%)\end{array}$ & - & - & - & 15.00 & 30.00 & 60.00 \\
$\begin{array}{c}\text { Spray dried lactose } \\
(\%)\end{array}$ & 54.00 & 39.00 & 9.00 & 54.00 & 39.00 & 9.00 \\
$\begin{array}{c}\text { Ac-Di-Sol (\%) } \\
\text { Total (\%) }\end{array}$ & 1.00 & 1.00 & 1.00 & 1.00 & 1.00 & 1.00 \\
\hline
\end{tabular}


Table 3-2. Formulations for effect of physical mixing on furosemide particles

\begin{tabular}{ccccccc}
\hline Ingredients & F-20 & F-21 & F-22 & F-23 & F-24 & F-25 \\
\hline $\begin{array}{c}\text { Furosemide (\%) } \\
\text { Lutrol F68 micro } \\
(\%)\end{array}$ & 10.00 & 20.00 & 30.00 & 10.00 & 20.00 & 30.00 \\
$\begin{array}{c}\text { Spray dried lactose } \\
(\%)\end{array}$ & 89.90 & 79.90 & 69.90 & 84.90 & 74.90 & 64.90 \\
$\begin{array}{c}\text { Ac-Di-Sol (\%) } \\
\text { Total (\%) }\end{array}$ & 0.10 & 0.10 & 0.10 & 0.10 & 0.10 & 0.10 \\
\hline
\end{tabular}


Table 3-3. Formulations for effect of physical mixing of PEG 3350 on furosemide particles

\begin{tabular}{cc}
\hline Ingredients & F-28 \\
\hline Furosemide (\%) & 10.00 \\
PEG 3350 (\%) & 10.00 \\
Spray dried lactose (\%) & 79.90 \\
Ac-Di-Sol (\%) & 0.10 \\
Total (\%) & 100.00 \\
\hline
\end{tabular}


Table 3-4. Formulations for effect of roller compaction on griseofulvin particles

\begin{tabular}{ccccccc}
\hline Ingredients & F-7 & F-8 & F-9 & F-10 & F-11 & F-12 \\
\hline Griseofulvin (\%) & 30.00 & 30.00 & 30.00 & 30.00 & 30.00 & 30.00 \\
PEG 3350 (\%) & 15.00 & 30.00 & 60.00 & - & - & - \\
Lutrol F68 micro (\%) & - & - & - & 15.00 & 30.00 & 60.00 \\
Spray dried lactose (\%) & 54.00 & 39.00 & 9.00 & 54.00 & 39.00 & 9.00 \\
Ac-Di-Sol (\%) & 1.00 & 1.00 & 1.00 & 1.00 & 1.00 & 1.00 \\
Total (\%) & 100.00 & 100.00 & 100.00 & 100.00 & 100.00 & 100.00 \\
\hline
\end{tabular}


Table 3-5. Formulations for effect of physical mixing and roller compaction on furosemide particles

\begin{tabular}{ccc}
\hline Ingredients & F-26(Physical mixing) & F-27 (Roller compaction) \\
\hline Furosemide (\%) & 20.00 & 20.00 \\
Lutrol F68 micro (\%) & 20.00 & 20.00 \\
Spray dried lactose (\%) & 59.90 & 59.90 \\
Ac-Di-Sol (\%) & 0.10 & 0.10 \\
Total (\%) & 100.00 & 100.00 \\
\hline
\end{tabular}


Table 3-6. Formulations for effect of physical mixing of mannitol on furosemide particles

\begin{tabular}{cccc}
\hline Ingredients & F-13 & F-14 & F-15 \\
\hline Furosemide (\%) & 10.00 & 20.00 & 30.00 \\
Mannitol SD 200 (\%) & 89.90 & 79.90 & 69.90 \\
Ac-Di-Sol (\%) & 0.10 & 0.10 & 0.10 \\
Total (\%) & 100.00 & 100.00 & 100.00 \\
\hline
\end{tabular}


Table 3-7. Formulations for effect of spray drying with mannitol on furosemide particles

\begin{tabular}{cccc}
\hline Ingredients & F-16 & F-17 & F-18 \\
\hline Furosemide (\%) & 10.00 & 20.00 & 30.00 \\
Mannitol SD 200 (\%) & 89.90 & 79.90 & 69.90 \\
Ac-Di-Sol (\%) & 0.10 & 0.10 & 0.10 \\
Total (\%) & 100.00 & 100.00 & 100.00 \\
\hline
\end{tabular}




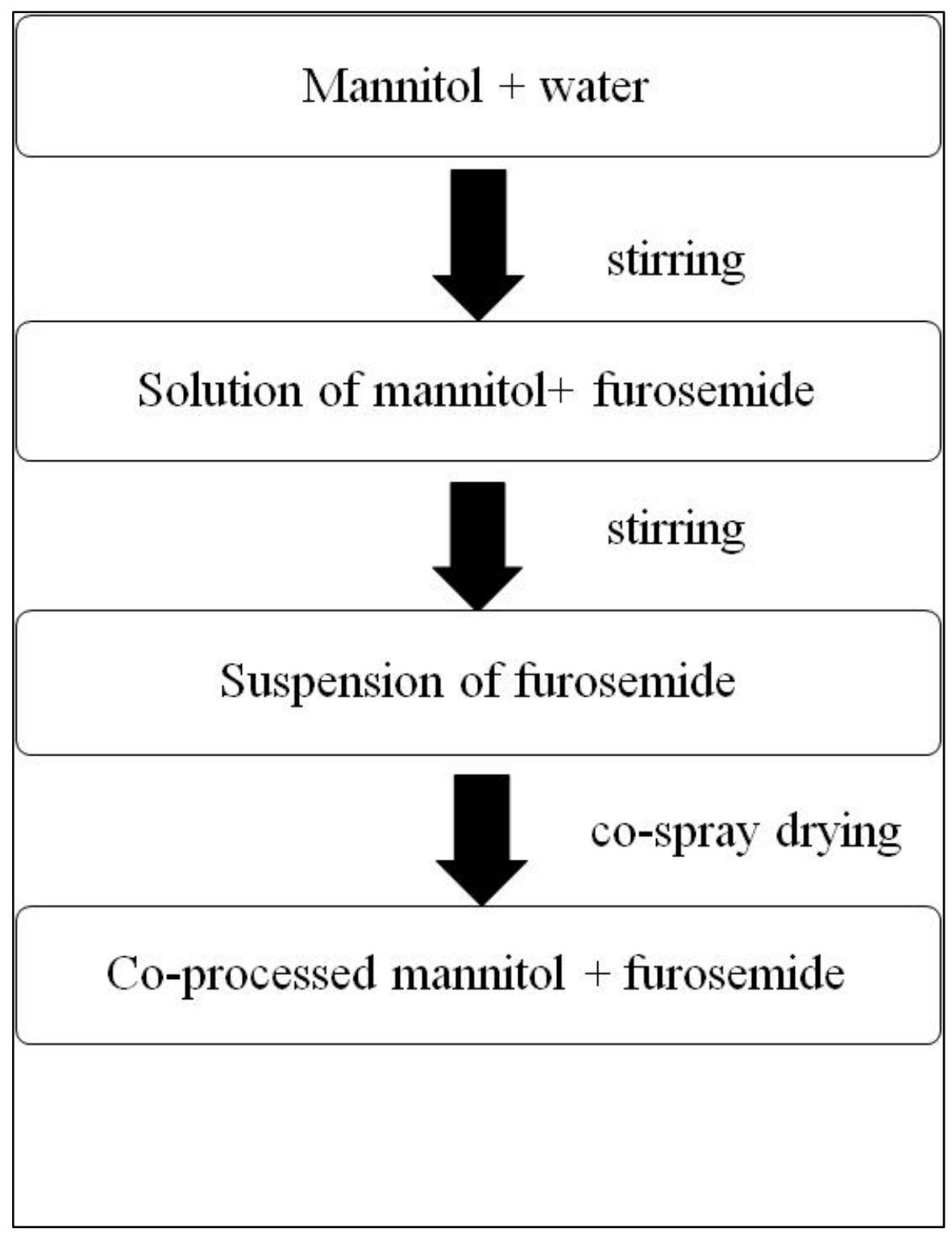

Figure 3-1. Preparation procedure for co-processed powder of mannitol and furosemide 
Furosemide and Lycoat RS 720suspension was spray dried at $100^{\circ} \mathrm{C}$ inlet and $80^{\circ} \mathrm{C}$ outlet temperature; aspirator capacity was $100 \%$ and feed rate of $20 \%$ and $60 \mathrm{ml} / \mathrm{hr}$ airflow. The co-processed powder was collected in cyclone separator and blended with remaining excipients presented in Table 3-8. The final blend was compressed into tablets at various compression forces. Procedure for preparation of co-processed powder is presented in Figure 3-2.

\subsubsection{Effect of level of super disintegrant}

The effect of the level of super disintegrant was evaluated for preventing the agglomeration of API during compression. Micronized furosemide and spray dried lactose monohydrate were accurately weighed by varying the Ac-Di-Sol levels according to Table 3-9. Initially furosemide and spray dried lactose monohydrate were geometrically mixed together on paper and by passing through \#60 stainless steel mesh twice. Finally Ac-Di-Sol was blended in a plastic bag for 5 min. the final blend was compressed into tablets at various compression forces. The compressed tablets were disintegrated in a furosemide saturated medium and particle size of furosemide after disintegration was estimated by light scattering technique. Saturated furosemide solution was used as medium for particle size measurement. Six portions of blend (200 $\mathrm{mg}$ each) were individually weighed and compressed for each level of compression force.

\subsubsection{Effect of hexane treatment}

Based on the formulation micronized furosemide and spray dried lactose monohydrate were accurately weighed and dispersed in $150 \mathrm{ml}$ of hexane and stirred using magnetic stirrer for 4 hours to make a slurry (furosemide and lactose were insoluble in hexane). The slurry was dried while stirring under fume hood in a crucible. The dried residue was further dried under vacuum oven at $60^{\circ} \mathrm{C}$ for 24 hours to get rid of any residual hexane. The residue was characterized for surface morphology by using Scanning Electron Microscopy. Final blend was prepared based on the formulation in Table 3-10.

\subsubsection{Compression of tablets}

Tablets of weight $200 \mathrm{mg}$ for furosemide and $400 \mathrm{mg}$ for griseofulvin were compressed on a Carver hydraulic manual bench top laboratory press (Model C, Carver, Inc) with dual scale gauge. 5/16" FFBE (flat face bevel edge) for furosemide and 3/8" FF (flat face) for griseofulvin tooling were used with $7.93 \mathrm{~mm}$ and $9.5 \mathrm{~mm}$ diameter tooling was used respectively. Lubricant, magnesium stearate was applied externally to the faces of upper and lower punches before each tablet compression. 
Table 3-8. Formulations for effect of spray drying with Lycoat RS 720 on furosemide particles

\begin{tabular}{cc}
\hline Ingredients & F-19 \\
\hline Furosemide (\%) & 30.00 \\
Lycoat RS 720 (\%) & 1.00 \\
Mannitol SD 200 (\%) & 68.90 \\
Ac-Di-Sol (\%) & 0.10 \\
Total (\%) & 100.00 \\
\hline
\end{tabular}




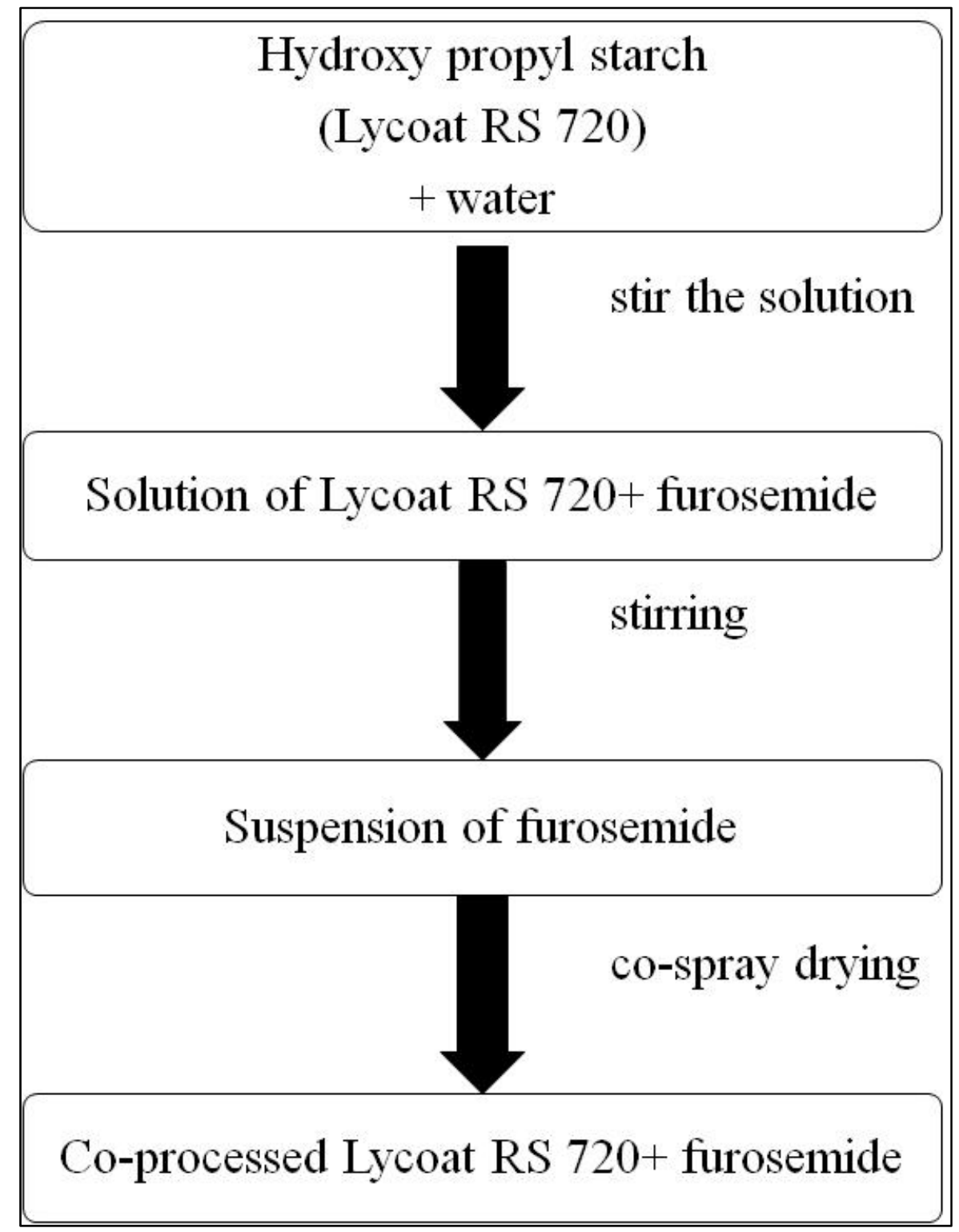

Figure 3-2. Preparation procedure for co-processed furosemide and Lycoat RS 720 powder 
Table 3-9. Formulations for level of super disintegrant

\begin{tabular}{cccc}
\hline Ingredients & F-29 & F-30 & F-31 \\
\hline Furosemide (\%) & 30.00 & 30.00 & 30.00 \\
Spray dried lactose (\%) & 69.90 & 69.00 & 68.00 \\
Ac-Di-Sol (\%) & 0.10 & 1.00 & 2.00 \\
Total (\%) & 100.00 & 100.00 & 100.00 \\
\hline
\end{tabular}


Table 3-10. Formulation for hexane treatment effect for furosemide

\begin{tabular}{cc}
\hline Ingredients & F-32 \\
\hline Furosemide (\%) & 30.00 \\
Spray dried lactose (\%) & 69.90 \\
Ac-Di-Sol (\%) & 0.10 \\
Total (\%) & 100.00 \\
\hline
\end{tabular}




\subsubsection{Disintegration of tablets}

Disintegration of tablets was done in QC-21 ${ }^{\mathrm{TM}}$ disintegration test system in saturated drug solution at room temperature. The disintegration was continued further for another one hour after disintegration of tablets to dissolve all soluble excipients in the media. The residue was collected by vacuum filtration unit with $0.45 \mu \mathrm{m}$ nylon filters and proceeds further for particle size measurement and characterization.

\subsubsection{Particle size analysis}

A Saturn Digisizer ${ }^{\circledR} 5200$, a high resolution laser particle size analyzer attached with low volume liquid sampling handling unit, was used to measure the particle size of the residue after the disintegration study. The low volume LSHU holds only $100 \mathrm{ml}$ of saturated medium for carrying the sample and the circulation pump rate was adjusted to $6 \mathrm{~L}$ per minute. Each sample was analyzed three times. Saturated furosemide solution for furosemide samples and saturated griseofulvin solution for griseofulvin samples was used as dispersion media to measure the particle size. A small suspension sample was prepared in saturated drug solution to measure the particle size of the drug residue obtained after disintegration process. Particle size data obtained after compression at various compression forces and treatments were subjected to a student $t$ test and $p$-values were presented.

\subsubsection{Modulated differential scanning calorimetry}

Modulated Differential Scanning Calorimetric (MDSC) studies were carried out to confirm the polymorphic changes in furosemide and griseofulvin in the samples during processing and tablet compression using a Q2000 DSC (TA instruments). The data were analyzed using Universal Analysis software. Thermograms reverse heat flow was plotted against temperature.

\subsubsection{Scanning electron microscopy studies}

Surface morphologies of particles were studied using an environmental scanning electron microscope (SEM). SEM evaluation was conducted by spreading samples onto double-sided adhesive carbon tape on an aluminum stub. A thin coating $(\sim 25 \mathrm{~nm})$ of gold was applied on to the sample using a Hummer VII Sputtering System and the coated sample stubs were mounted and focused under ESEM XL 30 (FEI). All the samples were observed at an energy of $30 \mathrm{KV}$ and various magnifications. 


\subsection{Results and Discussion}

\subsubsection{Effect of physical mixing}

Effect of physical mixing of griseofulvin and Particle Agglomeration Inhibitors (PEG 3350/Lutrol F68 micro) at various ratios' on micronized griseofulvin particles during compression is presented in Figures 3-3 and 3-4. Both the PAI's showed decrease in the particle agglomeration of griseofulvin as the ratio of GSF to PAI decreases when compared to without PAI. The micronized griseofulvin particles with mean particle size of $13.96 \pm 0.23 \mu \mathrm{m}$ were increased to $55.00 \pm 7.2 \mu \mathrm{m}$ and $111.46 \pm 5.7 \mu \mathrm{m}$ without PAI at $30 \%$ drug loading compressed at 1.0 and $2.0 \mathrm{mT}$ forces respectively. After physical treatment with PEG 3350 in F-1(1:0.5), F-2( 1:1) and F-3 (1:2) formulations, the micronized griseofulvin particles agglomerates with mean particle size of $46.59 \pm 2.28$ $\mu \mathrm{m}, 60.64 \pm 1.33 \mu \mathrm{m}$ and $50.99 \pm 2.35 \mu \mathrm{m}$ at $1 \mathrm{mT}$ compression force and $72.06 \pm 2.49$ $\mu \mathrm{m}, 68.25 \pm 1.46 \mu \mathrm{m}$ and $60.13 \pm 2.13 \mu \mathrm{m}$ at $2 \mathrm{mT}$ compression force respectively with $30 \%$ drug loading.

Similarly by physical treatment with Lutrol F68 micro a nonionic block copolymer in the formulations F-4 (1:0.5), F-5 (1:1) and F-6 (1:2) decreased the agglomeration of micronized griseofulvin particles during compression at $1 \mathrm{mT}$ and $2 \mathrm{mT}$ compared to without PAI treatment. The mean particle size of griseofulvin after physical treatment with Lutrol F68 micro was found to be $37.05 \pm 2.56 \mu \mathrm{m}, 32.50 \pm 3.06 \mu \mathrm{m}$ and $21.88 \pm 0.91 \mu \mathrm{m}$ at $1 \mathrm{mT}$ compression force and $52.77 \pm 1.15 \mu \mathrm{m}, 37.17 \pm 2.51 \mu \mathrm{m}$ and $35.39 \pm 0.99 \mu \mathrm{m}$ at $2 \mathrm{mT}$ compression force respectively with $30 \%$ drug loading. Combination of properties like high surface area, low melting point and superior surface active properties of Particle agglomeration inhibitors (PEG 3350 and Lutrol F 68 micro) might be responsible in reducing the agglomeration of micronized griseofulvin particles during compression.

Shah et al. described the high energy ordered mixtures provide the more advantages than simple ordered mixtures in deaggregation of cohesive drugs by improving the efficiency of high surface area carrier and hydrophilic nature of lactose. ${ }^{114}$

Figure 3-5 represents the particle size of furosemide agglomerates formed during compression of formulations without PAI (F-20, F-21 and F-22) and with Lutrol F68 micro (F-23, F-24 and F-25). In Formulations F-23 to F-25, furosemide was physically mixed with Lutrol F68 micro (PAI) at decreasing ratios of drug to PAI (0.5 to 0.17). Micronized furosemide agglomeration during compression was decreased with decreasing ratios of drug to PAI. Treatment with Lutrol F68 micro, the $5.04 \mu \mathrm{m}$ sized furosemide was agglomerated only from to $10.82 \pm 3.1 \mu \mathrm{m}, 49.66 \pm 1.03 \mu \mathrm{m}$ and $89.52 \pm$ $3.79 \mu \mathrm{m}$ at furosemide to Lutrol ratio $0.5,0.25$ and 0.17 after compression at $2 \mathrm{mT}$ compression force respectively. The percent reduction in the agglomeration of micronized furosemide during compression in the presence of Lutrol F68 micro presented in the Figure 3-6. 


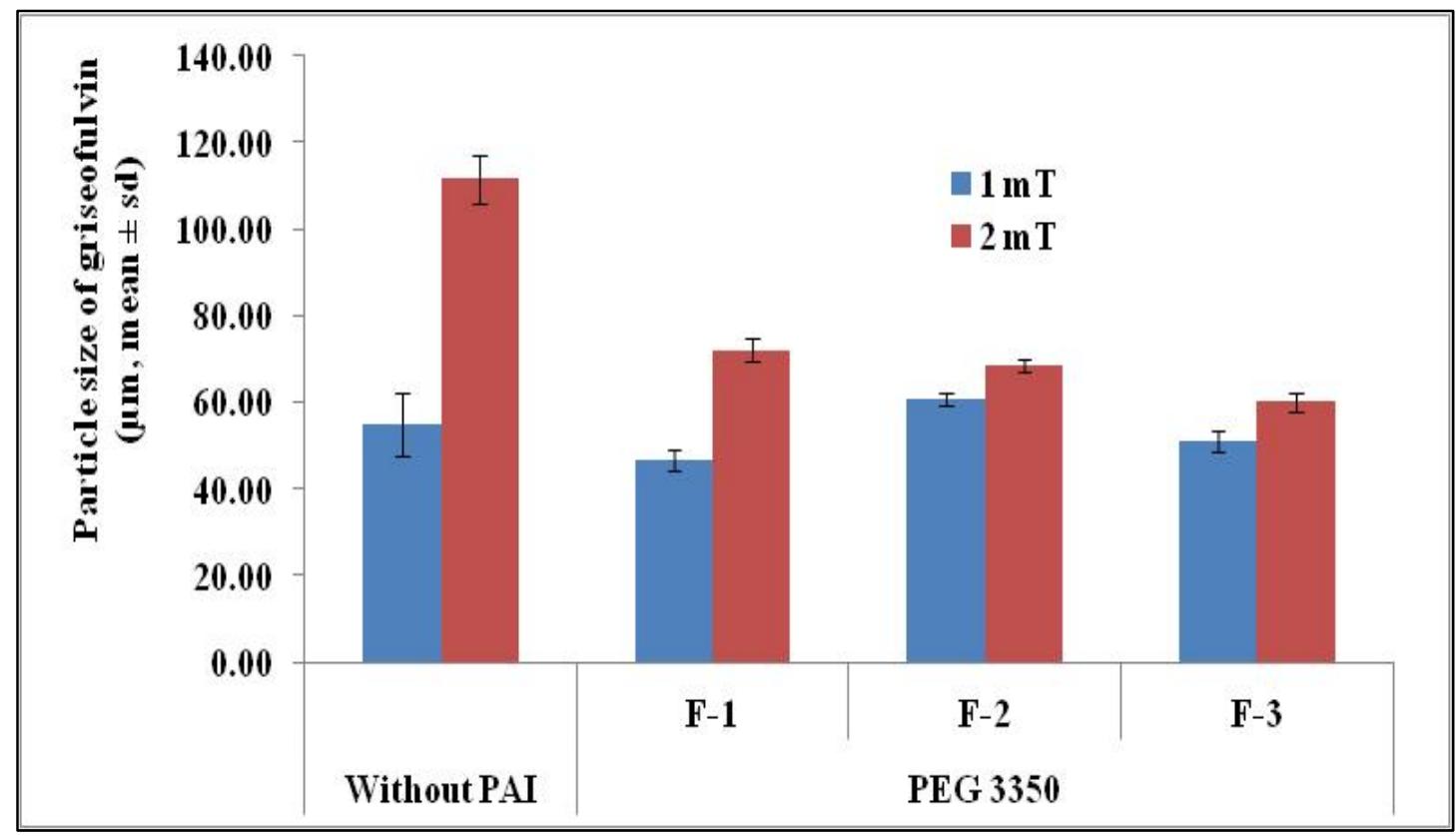

Figure 3-3. Effect of physical mixing with PEG 3350 on griseofulvin particles during compression 


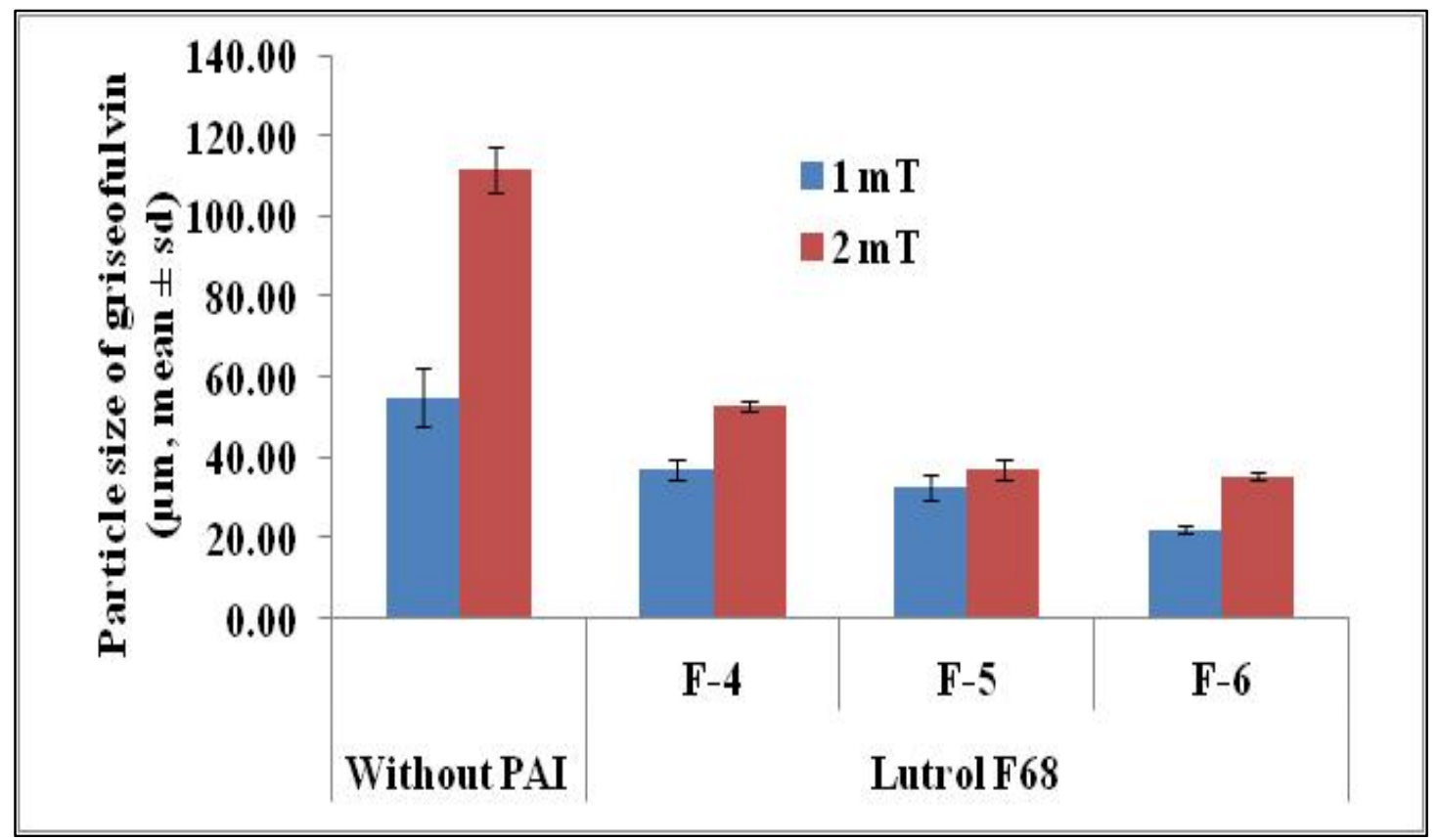

Figure 3-4. Effect of physical mixing with Lutrol F68 on griseofulvin particles during compression 


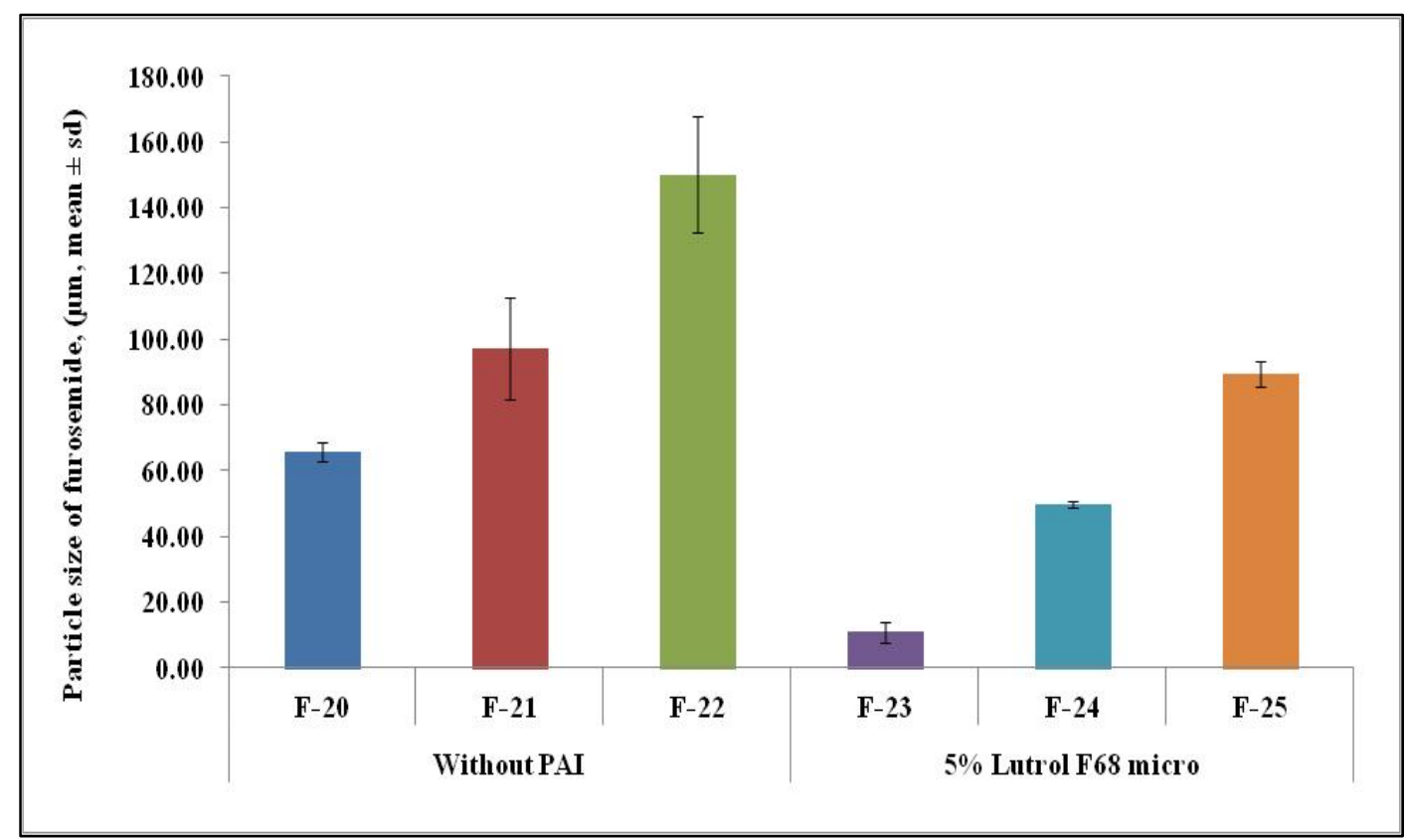

Figure 3-5. Effect of physical mixing of Lutrol F68 micro on furosemide particles during compression 


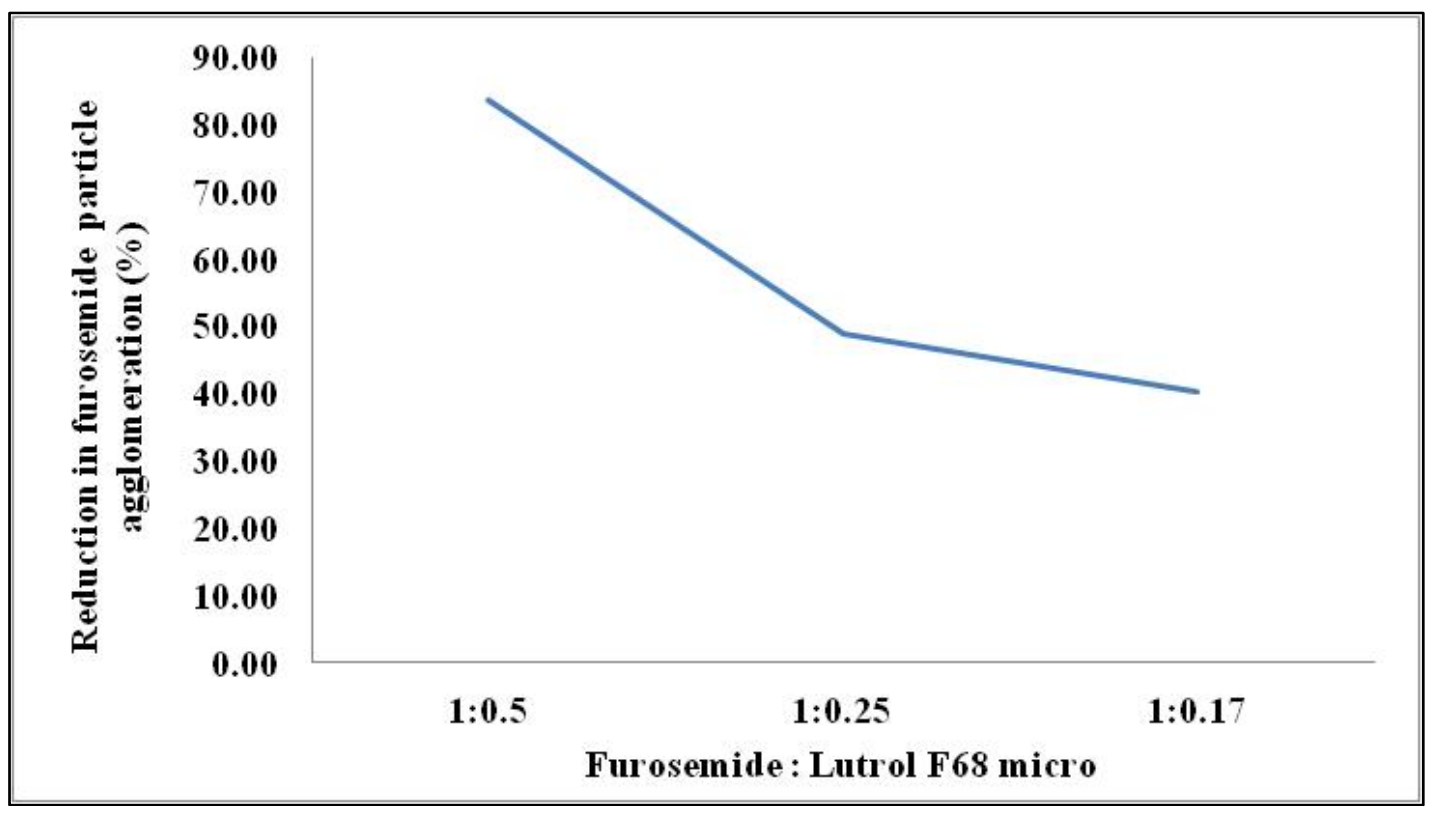

Figure 3-6. Effect of ratio of level of Lutrol F68 micro on agglomeration of furosemide particles during compression 
Prevention of agglomeration during compression of furosemide particles was achieved by physical treatment with PEG 3350 in formulation F-28 at 1:1 ratio of API to PAI and the mean particle size of agglomerates were $8.42 \mu \mathrm{m}$ and $21.09 \mu \mathrm{m}$ at $1 \mathrm{mT}$ and $2.0 \mathrm{mT}$ respectively.

\subsubsection{Effect of roller compaction}

SEM images of griseofulvin particles, after roller compaction treatment with Lutrol F68 micro and PEG 3350 are presented in Figure 3-7(a), (b) and (c) respectively. Effect of roller compaction of griseofulvin and Particle Agglomeration Inhibitors (PEG 3350/Lutrol F68 micro) at various ratios' on micronized griseofulvin particles during compression is presented in Figures 3-8 and 3-9. Both the PAI's showed decrease in the particle agglomeration of griseofulvin as the ratio of GSF to PAI decreases. The micronized griseofulvin particles with mean particle size of $13.96 \pm 0.23 \mu \mathrm{m}$ were increased to $55.00 \pm 7.2 \mu \mathrm{m}$ and $111.46 \pm 5.7 \mu \mathrm{m}$ with $30 \%$ drug loading at 1.0 and 2.0 $\mathrm{mT}$ compression forces respectively. After roller compaction treatment with PEG 3350 in F-7(1:0.5), F-8( 1:1) and F-9 (1:2) This is decreasing ratio of GSF to PAI formulations the micronized griseofulvin particles agglomerates with mean particle size of $74.17 \pm$ $6.10 \mu \mathrm{m}, 77.22 \pm 4.98 \mu \mathrm{m}$ and $29.68 \pm 0.96 \mu \mathrm{m}$ at $1 \mathrm{mT}$ compression force and $78.19 \pm$ $4.23 \mu \mathrm{m},, 79.99 \pm 3.34 \mu \mathrm{m}$ and $47.00 \pm 1.95 \mu \mathrm{m}$ at $2 \mathrm{mT}$ compression force respectively with $30 \%$ drug loading. The interference of point to point contact and the low melting point of PEG 3350 and its surface active properties might be responsible for reduction in the agglomeration of micronized griseofulvin particles during compression.

Similarly by roller compaction treatment with Lutrol F68 micro a nonionic block co-polymer in the formulations F-10 (1:0.5), F-11 (1:1) and F-12 (1:2) decreased the agglomeration of micronized griseofulvin particles during compression at $1 \mathrm{mT}$ and $2 \mathrm{mT}$ compared to without PAI. The mean particle size of griseofulvin after roller compaction treatment with Lutrol F68 micro was found to be $40.89 \pm 0.92 \mu \mathrm{m}, 53.93 \pm 2.04 \mu \mathrm{m}$ and $40.03 \pm 2.01 \mu \mathrm{m}$ at $1 \mathrm{mT}$ compression force and $62.28 \pm 1.46 \mu \mathrm{m}, 49.58 \pm 1.43 \mu \mathrm{m}$ and $32.00 \pm 1.02 \mu \mathrm{m}$ at $2 \mathrm{mT}$ compression force respectively with $30 \%$ drug loading.

Effect of physical treatment with very low percent (5\%) of Lutrol F68 micro for furosemide in prevention of agglomeration during compression was found to be successful. Agglomeration of micronized furosemide during compression at $1 \mathrm{mT}$ and $2 \mathrm{mT}$ forces for Formulations F-26(physical treatment) and F-27 (roller compaction treatment) at 1:1 ratio of API to PAI (Lutrol F68 micro) was found to be much less. The mean particle size of agglomerated furosemide in Figure 3-10 during compression was $21.88 \pm 5.6 \mu \mathrm{m}, 29.37 \pm 2.00 \mu \mathrm{m}$ and $8.97 \pm 3.02 \mu \mathrm{m}$ and $36.23 \pm 2.57 \mu \mathrm{m}$ at $1 \mathrm{mT}$ and $2 \mathrm{mT}$ forces respectively. This low melting point nature and more uniform spreading of Lutrol F68 micro during roller compaction might be responsible for preventing drug-drug agglomeration during compression process. 


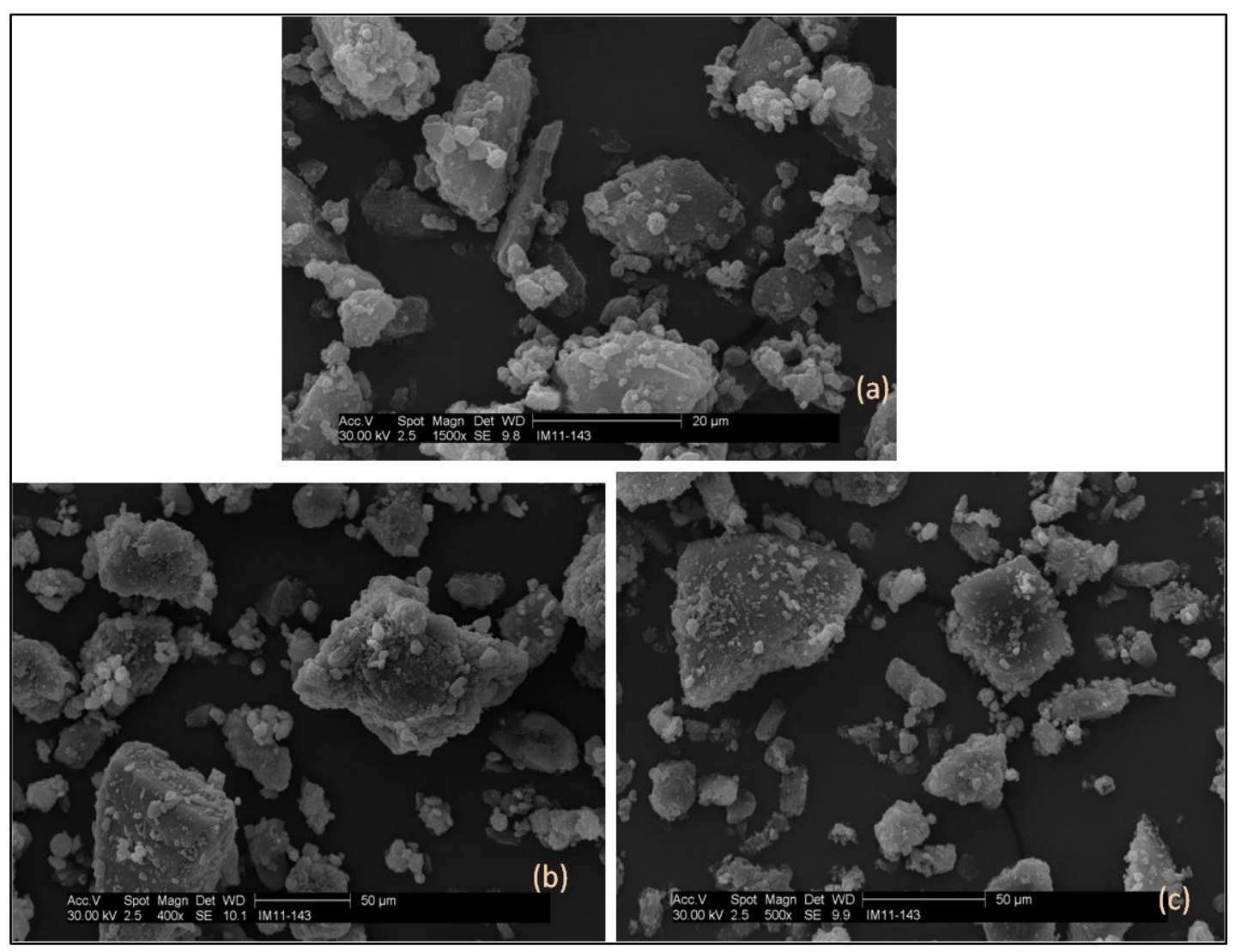

Figure 3-7. SEM image of (a) griseofulvin API particles (b) roller compacted griseofulvin + Lutrol F68 micro (c) roller compacted griseofulvin + PEG 3350 


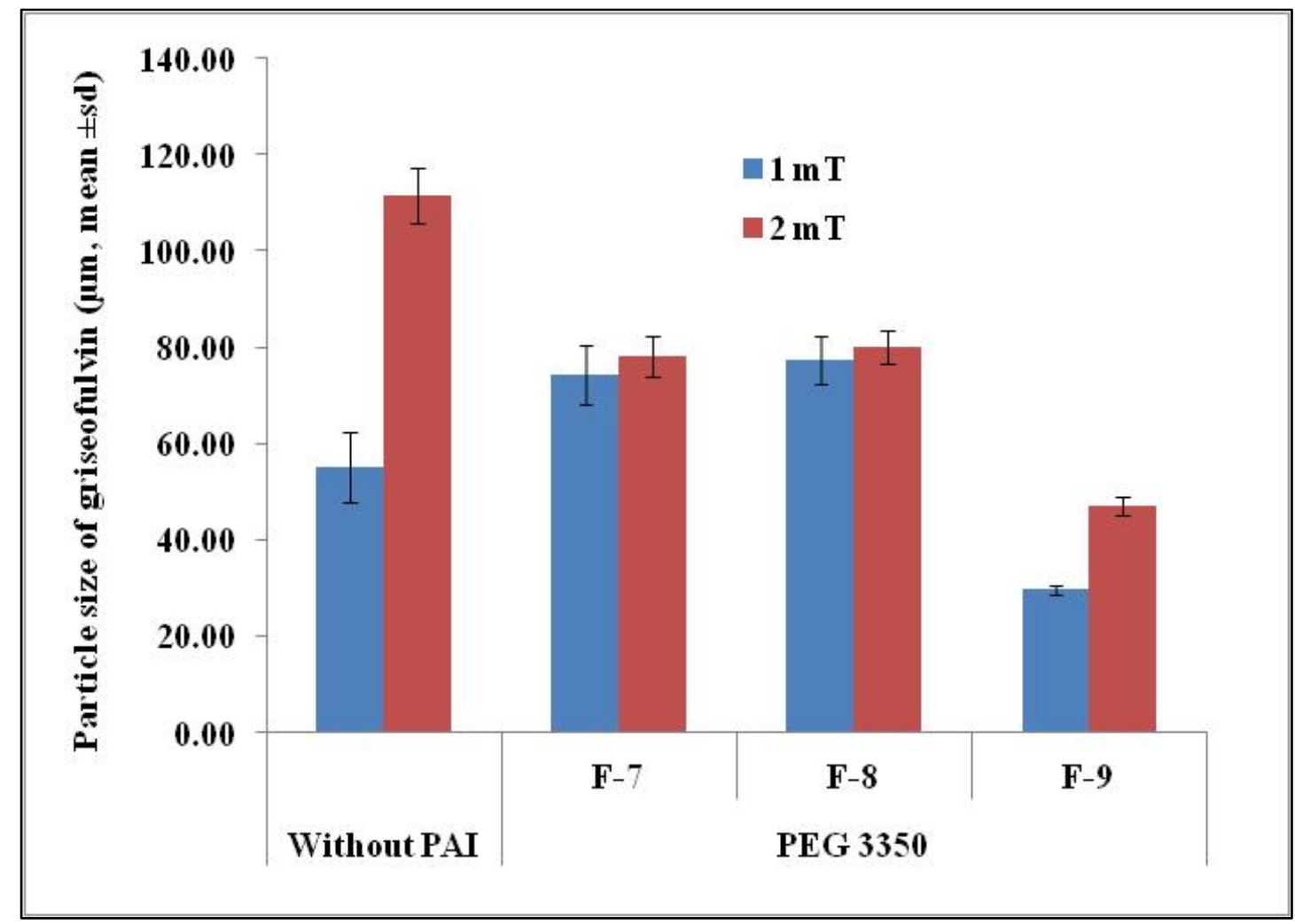

Figure 3-8. Effect of roller compaction with PEG 3350 on griseofulvin particles 


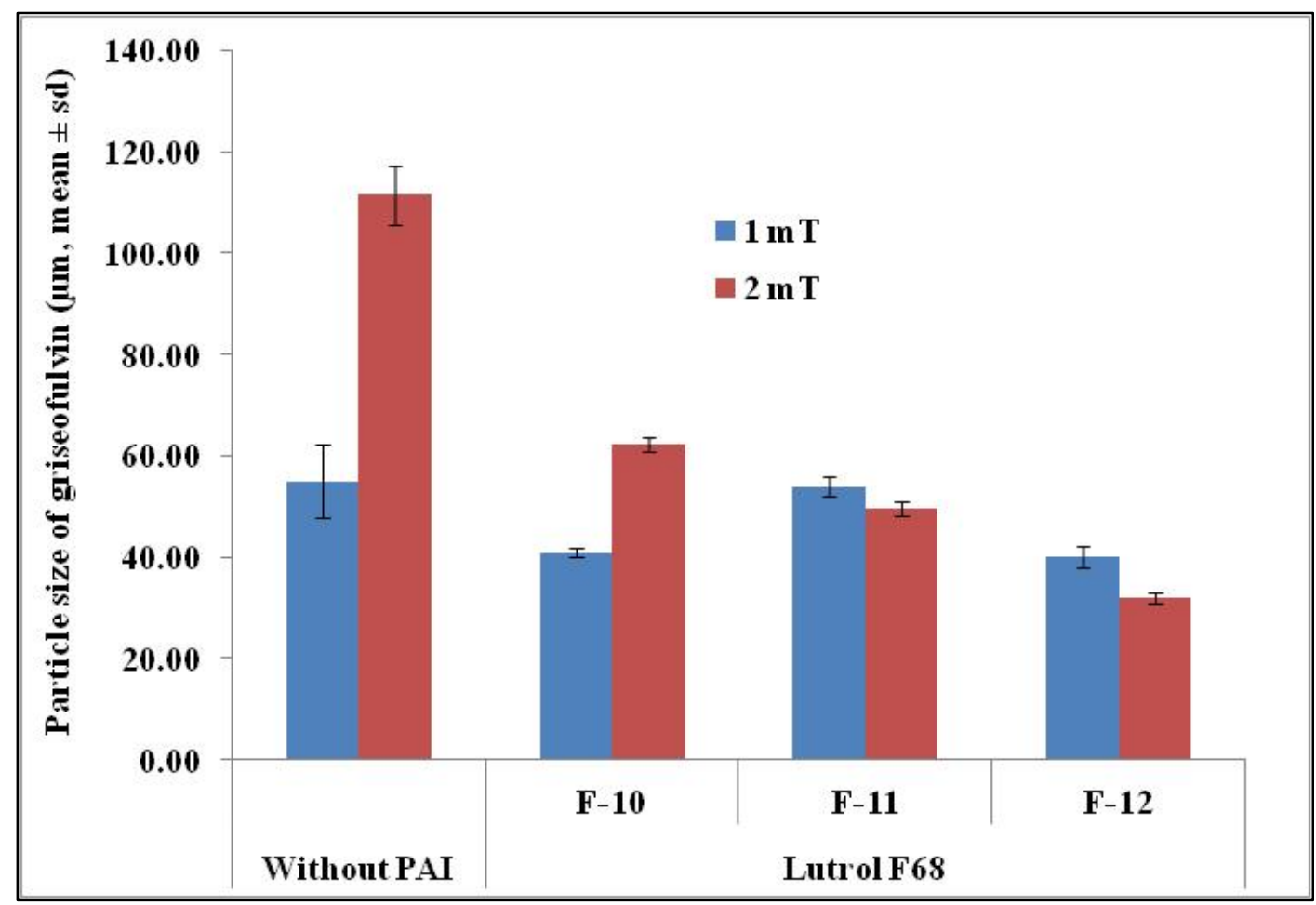

Figure 3-9. Effect of roller compaction with Lutrol F68 micro on griseofulvin particles 


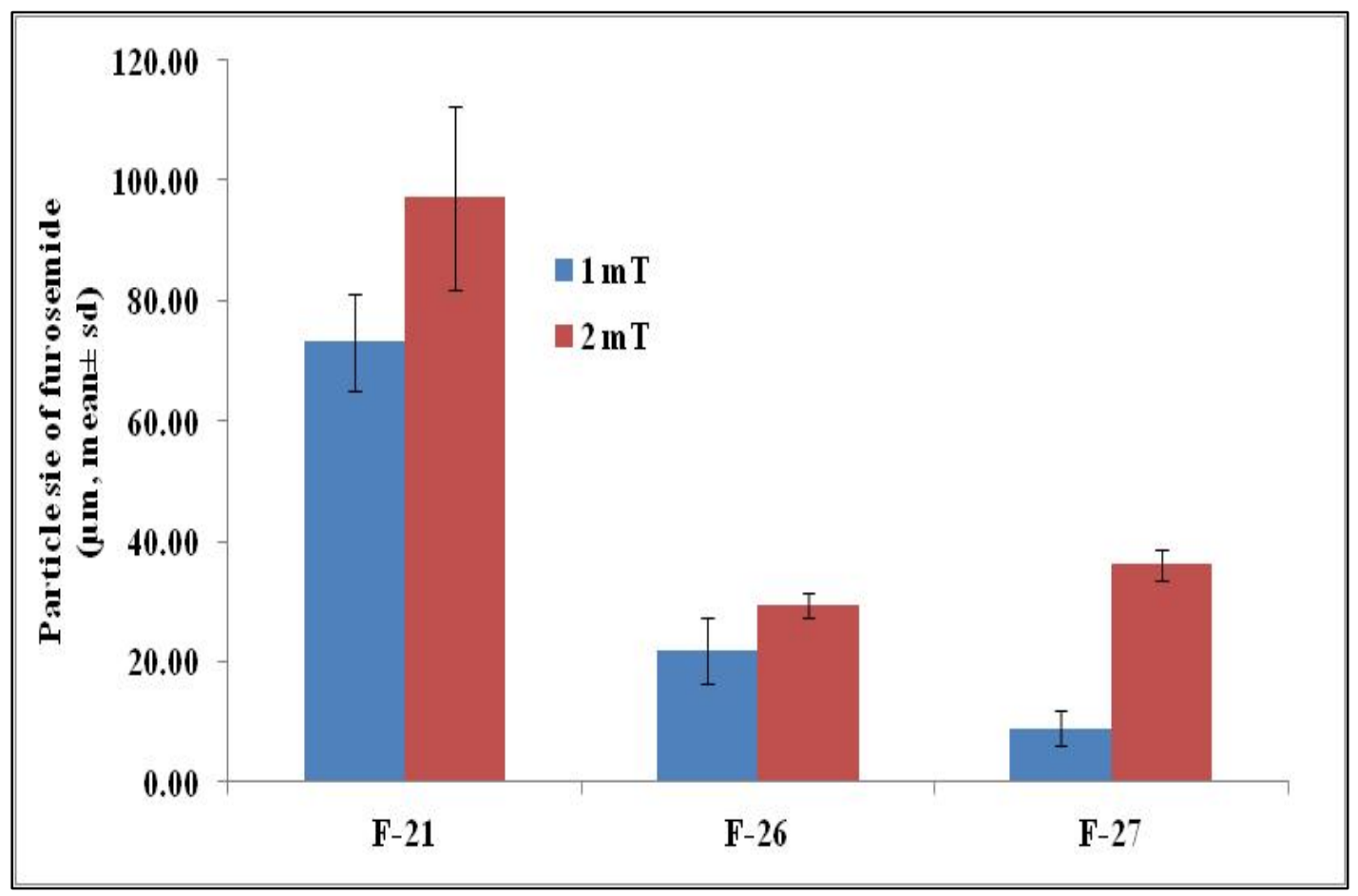

Figure 3-10. Effect of physical mixing and roller compaction with Lutrol F68 micro on furosemide particles during compression 


\subsubsection{Effect of spray drying}

\subsubsection{Mannitol SD 200}

Figure 3-11(a) and (b) represents the particle size distribution of furosemide before compression and after compression of formulation F-15 at $2 \mathrm{mT}$ respectively. The particle size distribution was represented in volume frequency percent. The mean particle size of furosemide before compression was found to be $5.04 \pm 0.5 \mu \mathrm{m}$. The size distribution curve for micronized furosemide showed a bi-modal distribution. Scanning electron microscopic images in Figure 3-12(a) represents micronized furosemide before compression. The effect of physical mixing with mannitol SD 200 on particle size of micronized furosemide during compression was evaluated by compressing the physical blend of furosemide at various compression forces for formulations F-13 (10\% drug loading), F-14 (20\% drug loading) and F-15 (30 \% drug loading). The micron size furosemide particles agglomerates into large chunks during compression with mean particle size range of $28.3 \pm 0.46,37.02 \pm 0.97,44.68 \pm 0.38$, and $46.77 \pm 1.83 \mu \mathrm{m}$ at 0.5 , $1.0,1.5$ and $2.0 \mathrm{mT}$ compression forces for formulation F-13 (10\% drug loading) respectively. For formulation F-14 with $20 \%$ drug loading these micronized furosemide particles agglomerates into $55.21 \pm 3.1,66.06 \pm 2.51,74.57 \pm 2.76$ and $83.05 \pm 2.97 \mu \mathrm{m}$ at $0.5,1.0,1.5$ and $2.0 \mathrm{mT}$ compression forces respectively. Similarly, for formulation F15 these drug particles agglomerates into $76.13 \pm 3.25,120.9 \pm 7.25,117.9 \pm 16.67$ and $128.7 \pm 7.51 \mu \mathrm{m}$. Figure 3-12(b) represents furosemide particles after compression at 2 $\mathrm{mT}$ for formulation F-15(30\% drug loading). Furosemide particles before compression were polygonal structure, cubical and rod in shape after compression these particles formed large agglomerates with narrow distribution shown in Figure 3-12. SEM image of furosemide was also correlating with the respective distributions before and after compression. Surface morphology of furosemide particles after compression was changed to rough, large chunks of agglomerates. Figure 3-13 represents effect of compression force and drug loading on furosemide particles with mannitol as a diluent by physical mixing.

Spray drying treatment of micronized furosemide with mannitol SD 200 reduced the agglomeration of furosemide particles during compression. SEM images in Figure 3-14(a) to (d) represents alone Mannitol SD 200 before spray drying, furosemide + Mannitol SD 200 particles after spray drying, micronized furosemide API particles before spray drying and after compression at $2 \mathrm{mT}$ of spray dried blend of furosemide + Mannitol SD 200 formulation (F-18) respectively. The spherical shape Mannitol SD 200 in Figure 3-14(a) did not altered its shape even after spray drying the suspension of furosemide in Figure 3-14(b). Spray drying with Mannitol SD 200 successfully reduced the agglomeration of micronized furosemide during compression for the formulations $\mathrm{F}$ 16 (10\% drug loading), F-17 (20\% drug loading) and F-18 (30\% drug loading). The mean particle size of agglomerated furosemide particles after compression of formulation F-16 was $8.44 \pm 0.63 \mu \mathrm{m}, 9.46 \pm 0.21 \mu \mathrm{m}, 10.24 \pm 0.29 \mu \mathrm{m}$ and $10.98 \pm 0.55 \mu \mathrm{m}$ at $0.5,1.0$, 1.5 and $2.0 \mathrm{mT}$ compression forces respectively. Compression of Formulation F-17 at $0.5,1.0,1.5$ and $2.0 \mathrm{mT}$ forces agglomerates into $24.51 \pm 0.78 \mu \mathrm{m}, 25.86 \pm 0.65 \mu \mathrm{m}$, 


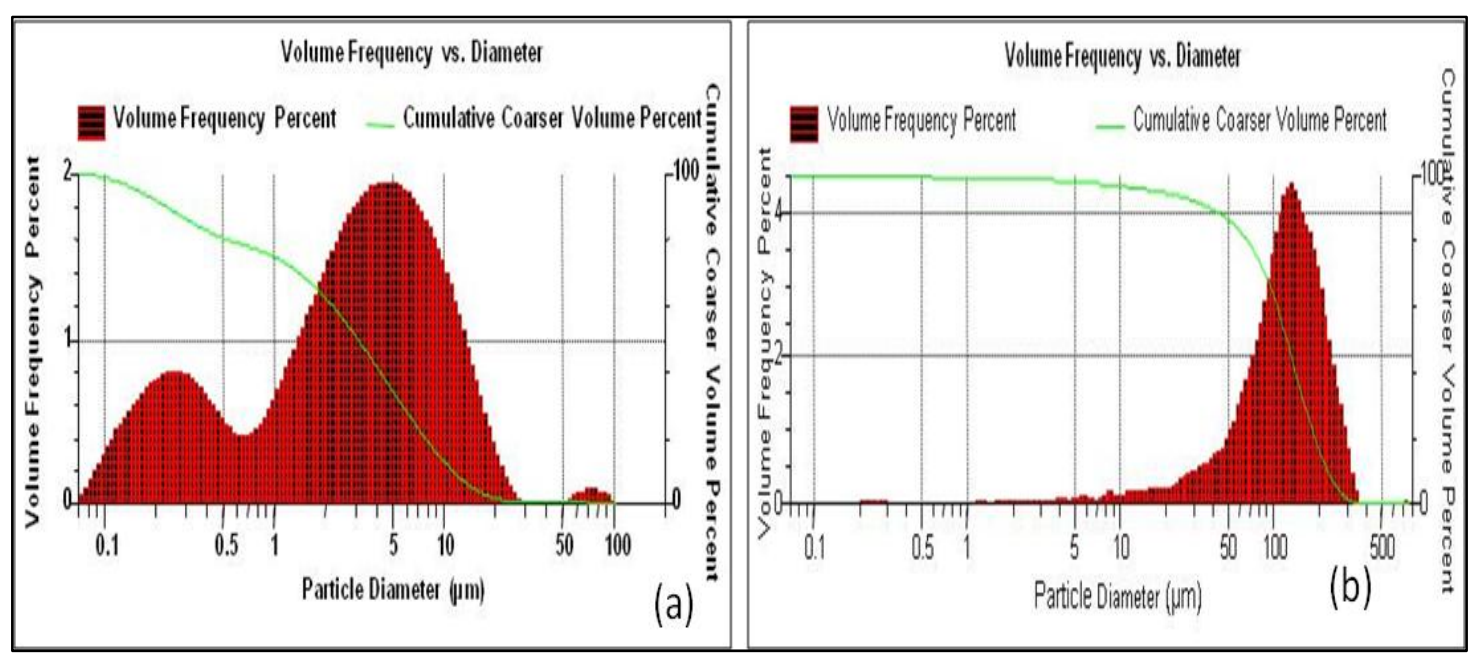

Figure 3-11. Particle size distribution of furosemide particles (a) before compression (b) after compression at $2 \mathrm{mT}$ with $30 \%$ drug loading 


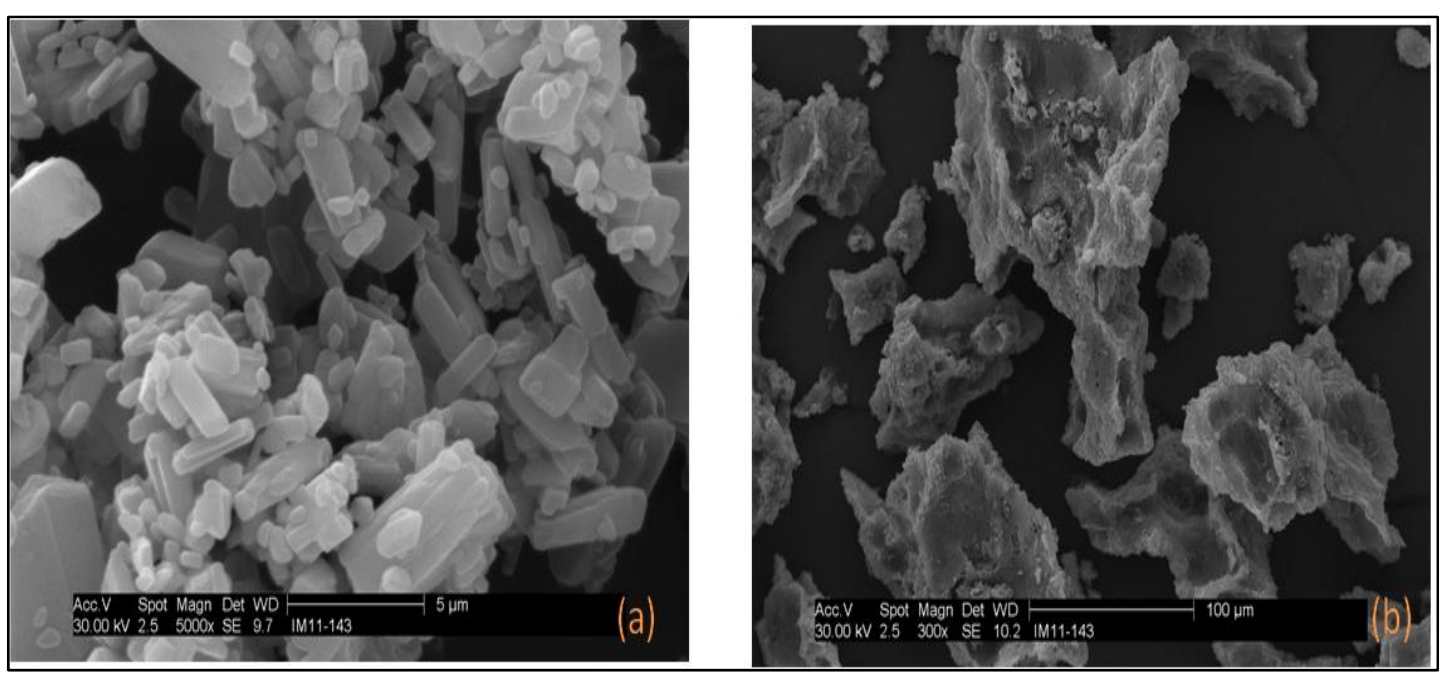

Figure 3-12. SEM image of furosemide particles (a) before compression (b) after compression (F-15) 


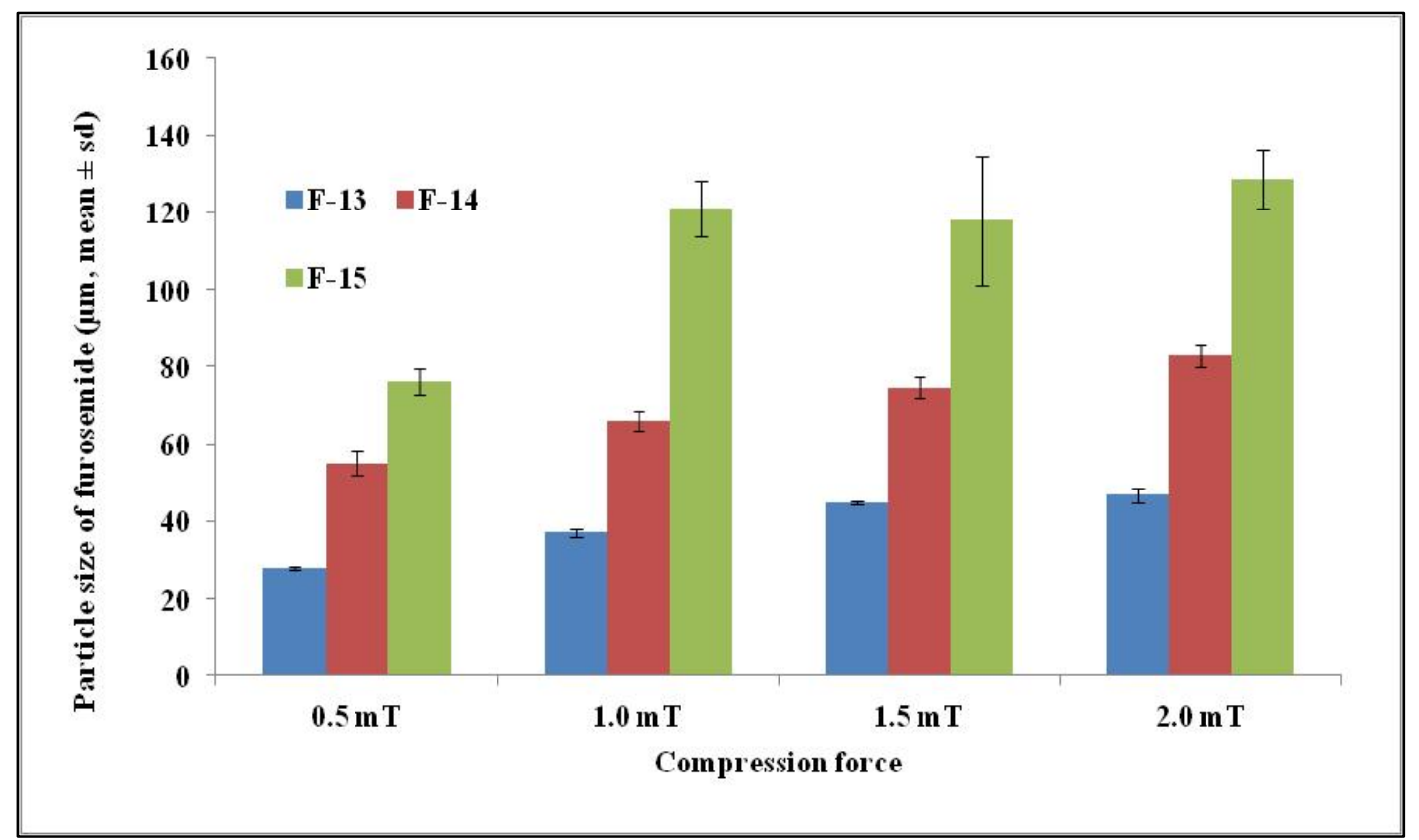

Figure 3-13. Effect of compression force and drug loading furosemide particles with mannitol as a diluent by physical mixing 


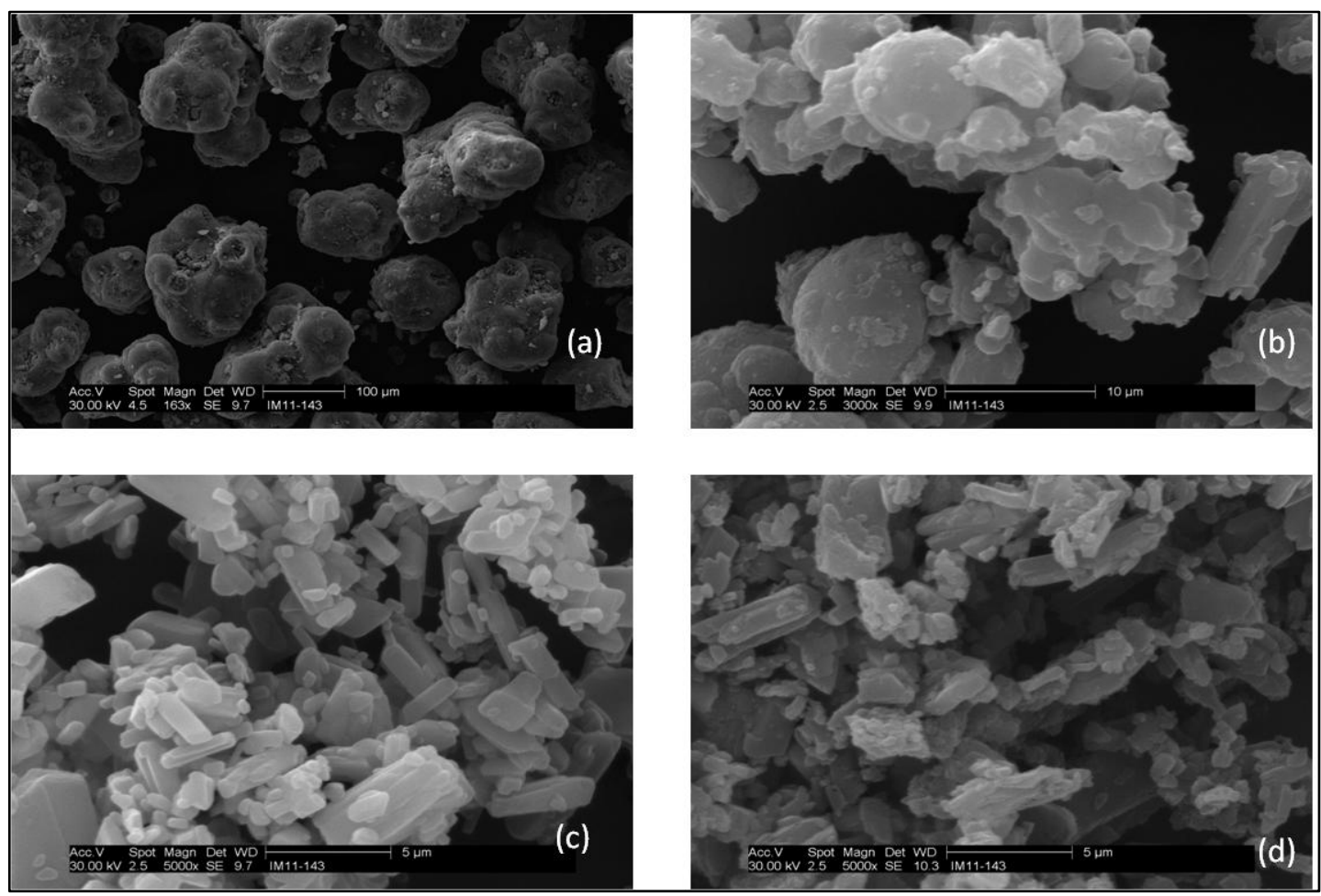

Figure 3-14. SEM image of (a) mannitol SD 200 particles (b) mannitol SD 200 + furosemide after spray drying (c) furosemide particles (d) furosemide particles after compression ( $F-18)$ 
$12.1 \pm 0.45 \mu \mathrm{m}$ and $20.83 \pm 4.11 \mu \mathrm{m}$ respectively. SEM image of furosemide particles after compression at $2 \mathrm{mT}$ for formulation F-18 is presented Figure 3-14(d). Similarly the Formulation F-18 aggregates into mean particle size of $29.4 \pm 5.95 \mu \mathrm{m}, 33.99 \pm 2.72 \mu \mathrm{m}$, $23.56 \pm 7.74 \mu \mathrm{m}$ and $28.26 \pm 1.16 \mu \mathrm{m}$ at $0.5,1.0,1.5$ and $2.0 \mathrm{mT}$ respectively. Figure 3-15 represents effect of compression force and drug loading on furosemide particles by spray drying process.

\subsubsection{Lycoat RS 720}

SEM image of furosemide in Figure 3-16 shows (a) pure API particles and (b) Spray dried sample of furosemide with hydroxy propyl starch (Lycoat RS 720). The images of spray dried mixture shows that micronized furosemide was coated by thin layer of hydroxy propyl starch which is specifically designed for aqueous film coating of immediate release solid dosage forms. Compression of blend containing coated mixture and remaining excipients in the formulation F-19 at $0.5,1.0,1.5$ and $2.0 \mathrm{mT}$ force results in the agglomerates of furosemide particle with mean particles size of $16.91 \pm 0.16 \mu \mathrm{m}$, $16.83 \pm 0.53 \mu \mathrm{m}, 15.27 \pm 0.23 \mu \mathrm{m}$ and $17.82 \pm 0.62 \mu \mathrm{m}$ respectively. Figure 3-17 represents effect of spray drying of furosemide with Lycoat RS 720 on furosemide particles during compression. Based on these results aqueous film coating by spray drying process with hydroxyl propyl starch + micronized furosemide particles successfully prevented the aggregation during compression. This might be due to fast dispersible nature and easy film formation in aqueous environment of hydroxy propyl starch.

\subsubsection{Effect of concentration of super disintegrant}

Incorporation of large percentage of super disintegrant in the formulation can prevent agglomeration of micronized drug particles during compression. However, the cost of the super disintegrant and formulation issues like stability, drug release needs to consider in adopting high percentage of super disintegrants in the formulation. The micronized furosemide particles in the formulations F-22, F-30 and F-31 agglomerated during compression with $30 \%$ drug loading and presented in the Figure 3-18. The mean particle size of formed agglomerates of furosemide with low levels of super disintegrant $(0.1 \%)$ were $139.5 \pm 6.8 \mu \mathrm{m}$ and $150.1 \pm 17.7 \mu \mathrm{m}$ at $1 \mathrm{mT}$ and $2 \mathrm{mT}$ compression force respectively. Similarly with increasing levels of super disintegrant $1.0 \%$ and $2 \%$ of AcDi-Sol in the formulations F-30 and F-31, the micronized furosemide particles agglomerated with the mean particle size of $61.58 \pm 4.11 \mu \mathrm{m}, 84.64 \pm 4.78 \mu \mathrm{m}$ and $64.1 \pm$ $3.5 \mu \mathrm{m}, 74.07 \pm 4.98 \mu \mathrm{m}$ at $1 \mathrm{mT}$ and $2 \mathrm{mT}$ compression force respectively. These results showing that increasing the concentration of super disintegrant in the formulation could not fully prevent the agglomeration of micronized furosemide particles during compression at $30 \%$ drug loading. However some particle agglomeration was prevented with increasing levels of super disintegrant. The capillary action of superdisintegrant at high concentration during disintegration of tablets might be responsible for this reduction in the particle size of agglomerates. 


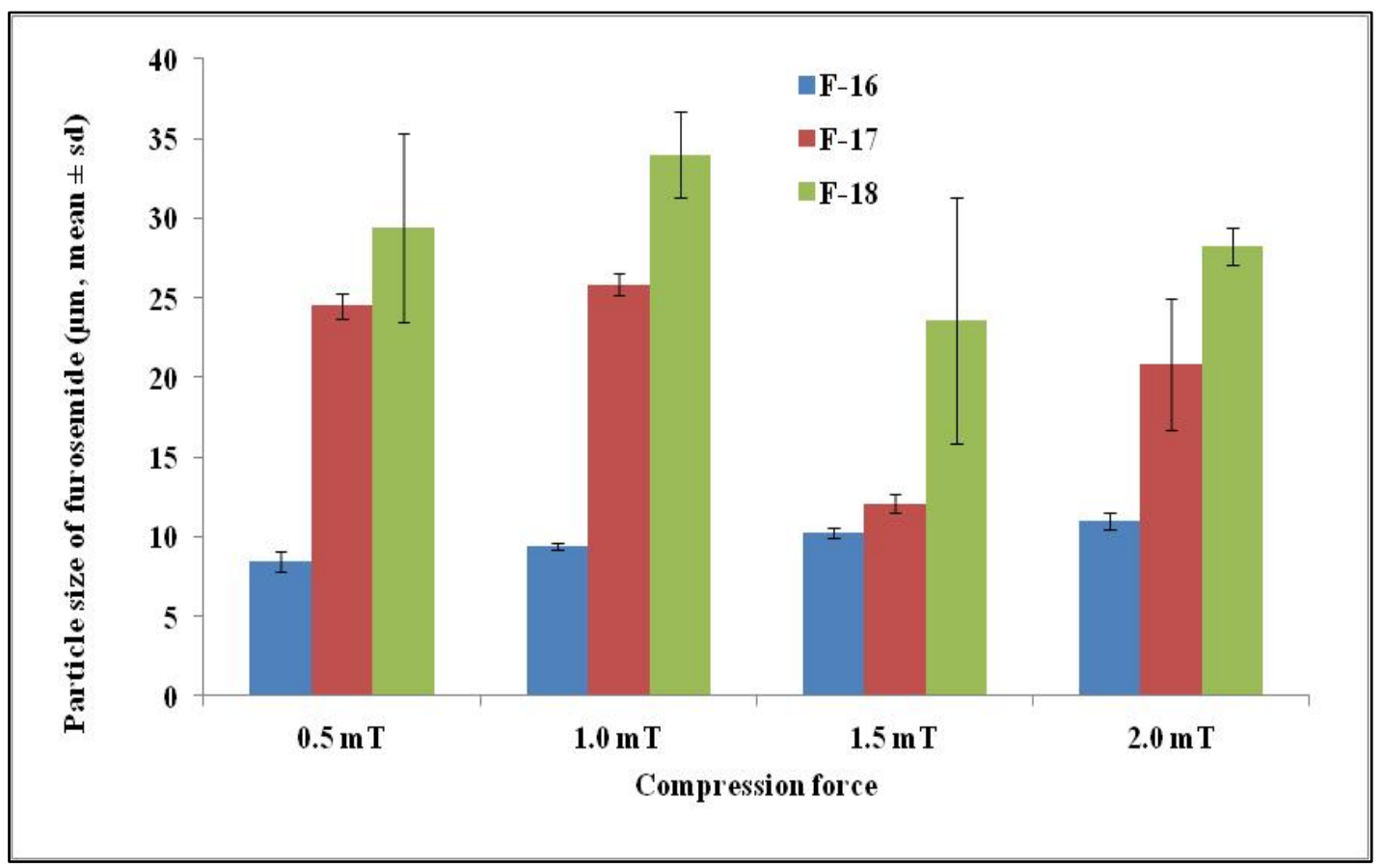

Figure 3-15. Effect of compression force and drug loading on furosemide particles by spray drying process 


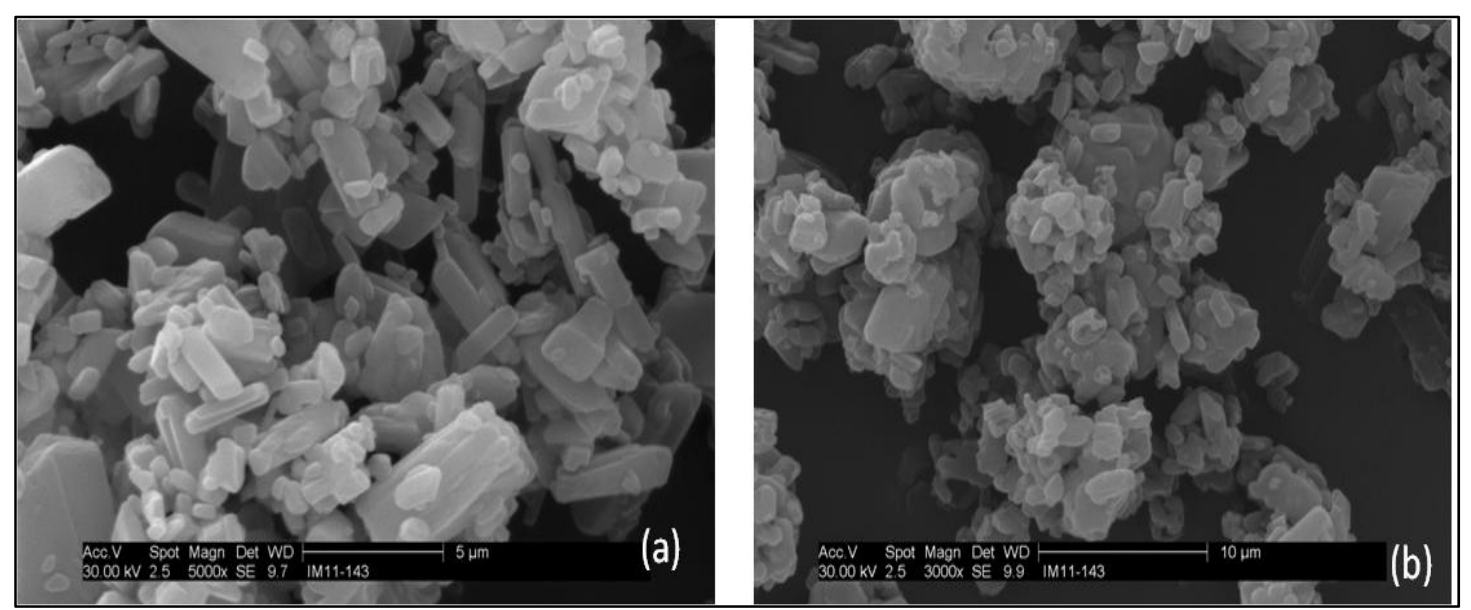

Figure 3-16. SEM image (a) furosemide particles (b) furosemide + Lycoat RS 720 spray dried particles 


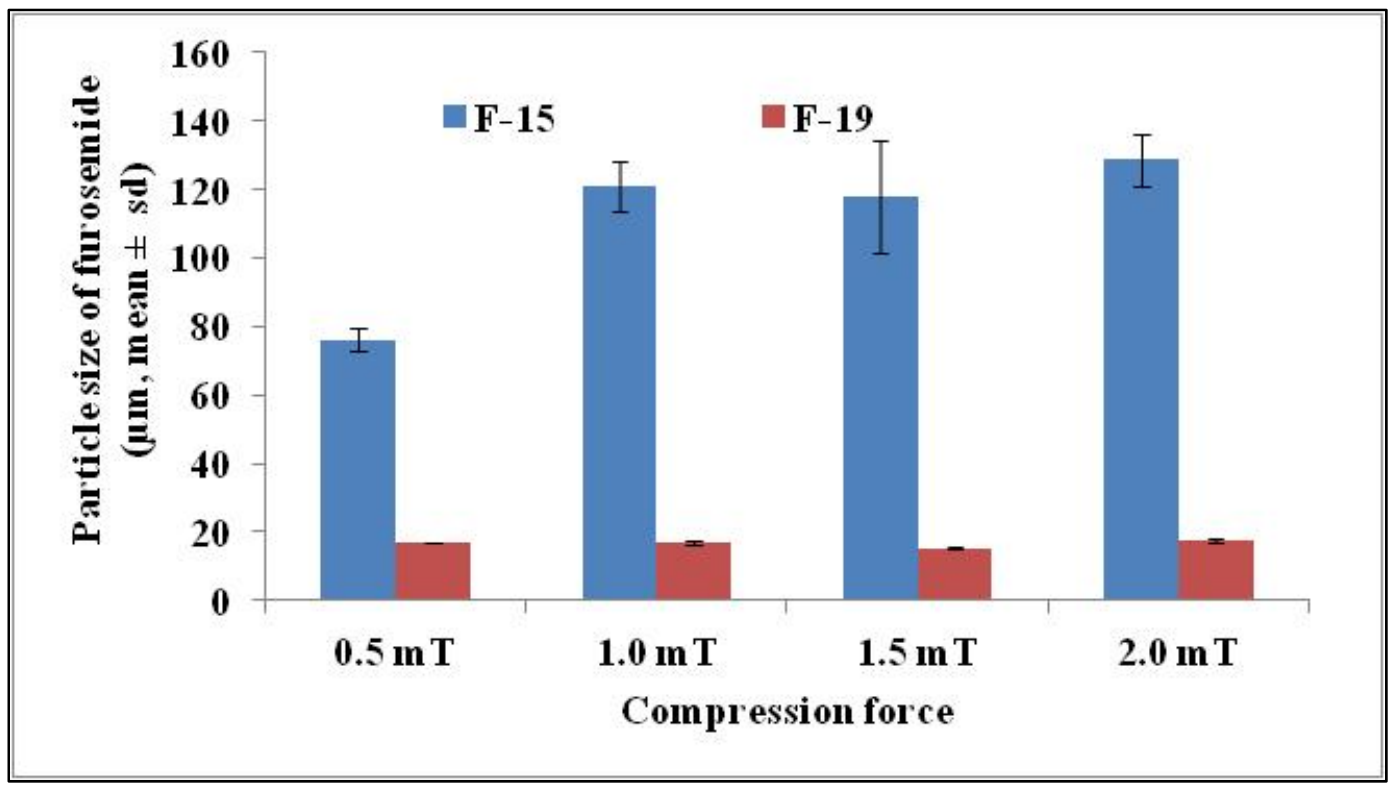

Figure 3-17. Effect of spray drying of furosemide with Lycoat RS 720 on furosemide particles during compression 


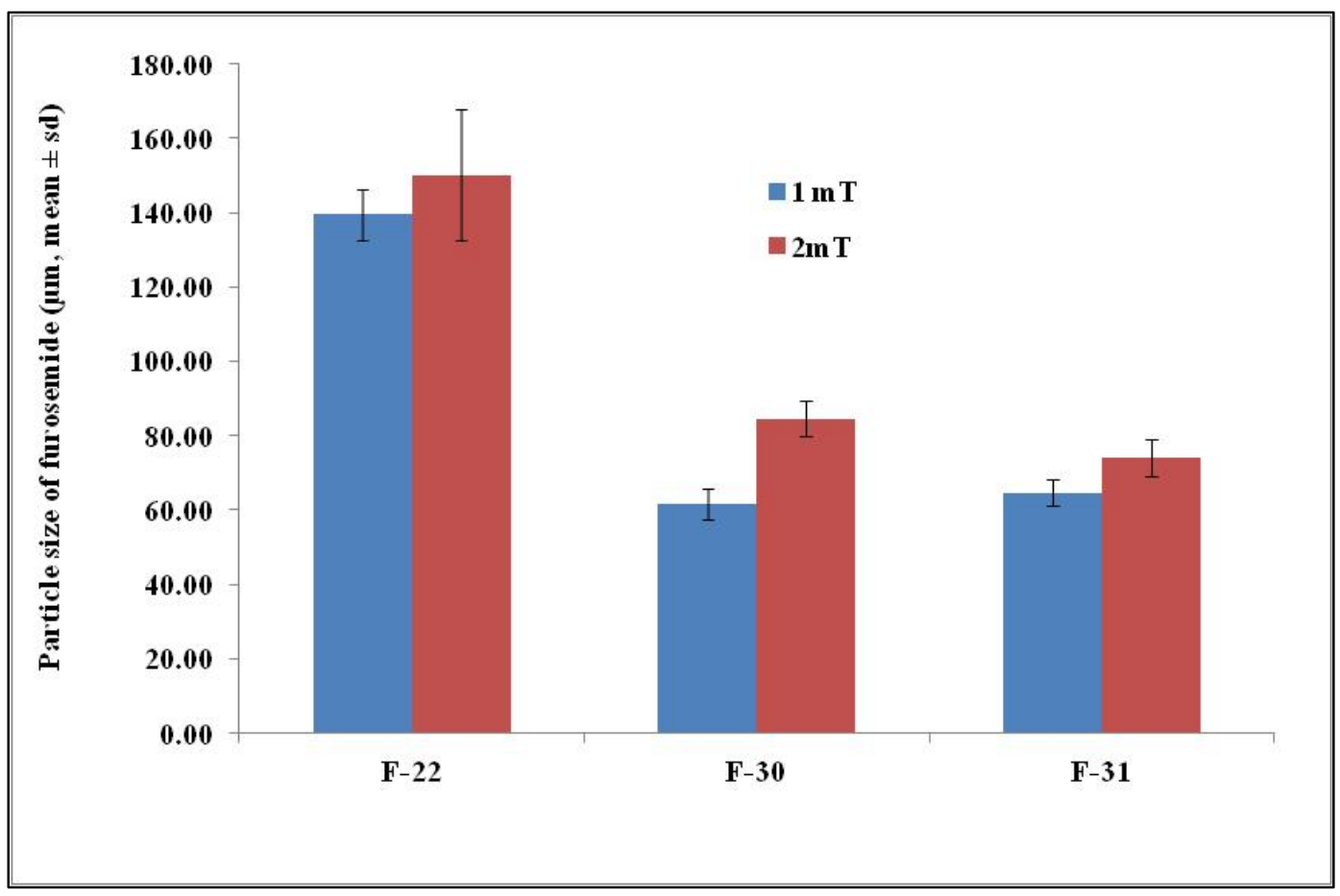

Figure 3-18. Effect of AC-Di-Sol concentration on furosemide particles during compression 


\subsubsection{Effect of hexane treatment}

Agglomeration of micronized furosemide during compression can be prevented by treating with Hexane. The SEM images in the Figure 3-19(a) to (c) shows furosemide coated spray dried lactose particles after hexane treatment at 400X, 200X and 6400X magnification respectively. The dried slurry of hexane shows uniform coating of micronized furosemide on large particles of spray dried lactose which could help in preventing drug-drug particle agglomeration during compression. Figure 3-20 shows the effect of hexane slurry treatment of furosemide particles after compression and disintegration. The mean particle size of agglomerates in formulation F-32 were found to be $56.70 \pm 5.42 \mu \mathrm{m}$ and $97.64 \pm 17.36 \mu \mathrm{m}$ with $30 \%$ drug loading at $1 \mathrm{mT}$ and $2 \mathrm{mT}$ force respectively. Hexane treatment helped some extent in preventing agglomeration during compression compared to without treatment (F-22). This might be due to uniform distribution of micronized furosemide particles on the large size spray dried lactose particles which might have created less contact area between drug particles during compression.

\subsubsection{Modulated differential scanning calorimetry}

Thermograms in the Figure 3-21 represent comparison of furosemide particles before compression and after compression. Treatment effect of on polymorphic changes in furosemide particles during compression was compared and a change in the melting point of furosemide was observed in physically treated and spray dried blends. This effect might be due to small portion of PAI traces in the sample or amorphous conversion of furosemide during treatment with PAI. Similarly the Figure 3-22 represents the thermograms of furosemide particles after compression at $2 \mathrm{mT}$ using the blend containing co-spray dried furosemide with Lycoat RS 720. Based on the MDSC results spray coating of Lycoat RS 720 (which is an aqueous film former) on furosemide particles did not show change any polymorphism of furosemide particles during compression. The sharp melting point at $210.52^{\circ} \mathrm{C}$ in reverse heat flow in the Figures 3-21 and 3-22 represents the crystalline nature of furosemide. Sharp endotherm at $195.31^{\circ} \mathrm{C}$ represents the melting point of Lycoat RS 720 in Figure 3-22, which is absent in the compressed and disintegrated tablets of blend containing Lycoat RS 720 spray coated furosemide particles. The compression of tablets was done at $2 \mathrm{mT}$ force with $30 \%$ drug loading. These thermograms were overlapping with the furosemide thermograms represent no polymorphic change during compression or spray drying. The MDSC thermograms in Figures 3-23 and 3-24 represent the effect of physical treatment and roller compaction treatment with particle agglomeration inhibitors (PEG 3350, Lutrol F68 micro) on griseofulvin particles during compression respectively.

The sharp endothermic peaks of griseofulvin at $218.58^{\circ} \mathrm{C}$ before and after compression at $2 \mathrm{mT}$ with 1:0.5 ratio of drug to PAI's shows a polymorphic change in griseofulvin. This melting endotherm corresponds to the crystalline nature of griseofulvin before and after compression but shift in the melting point might be due to impurities in the material or conversion to amorphous griseofulvin. 


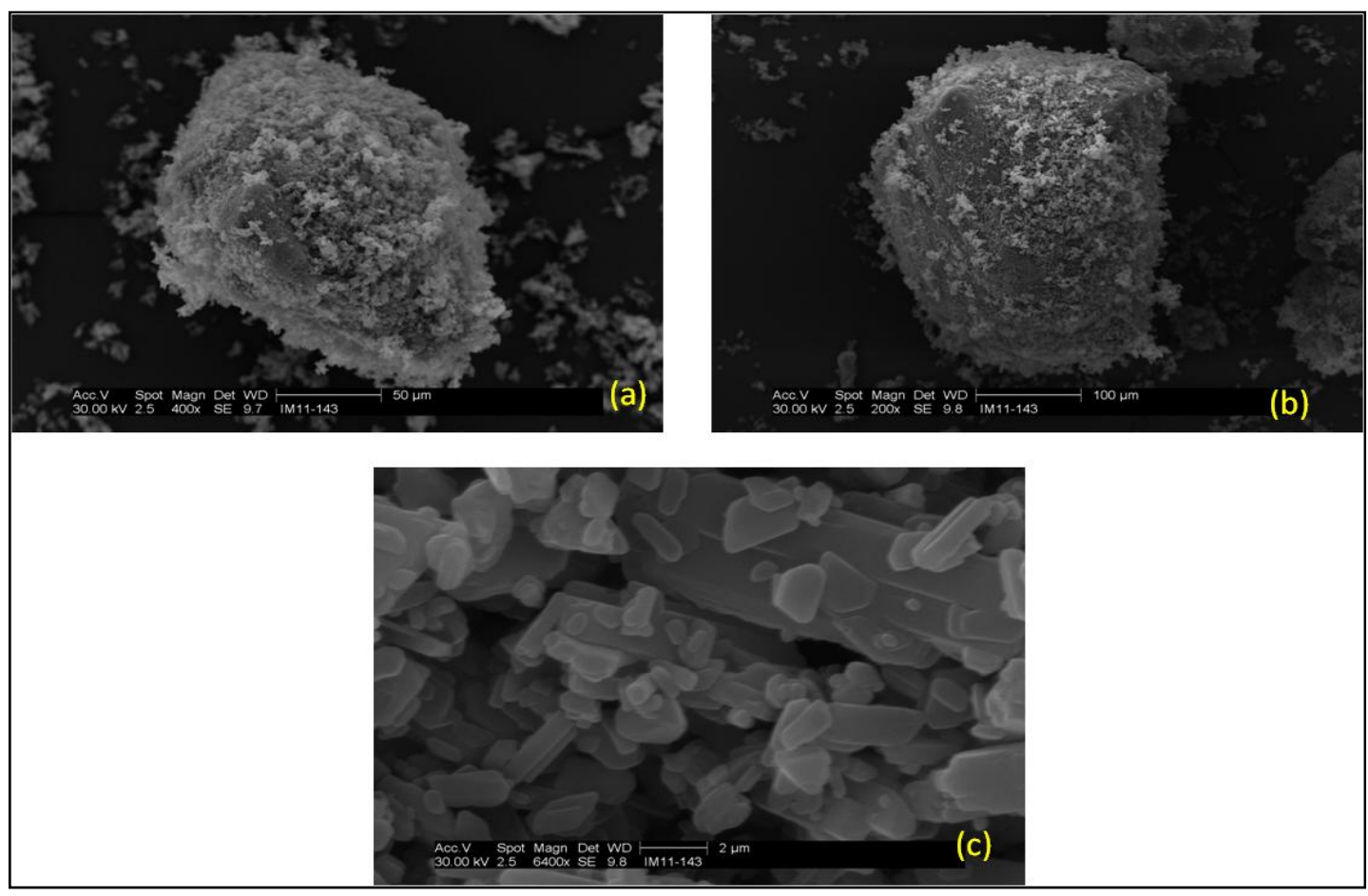

Figure 3-19. SEM images (a) 400X, (b) 200X, (c) 6400X magnification of furosemide particles coated on lactose monohydrate particle in hexane slurry treatment 


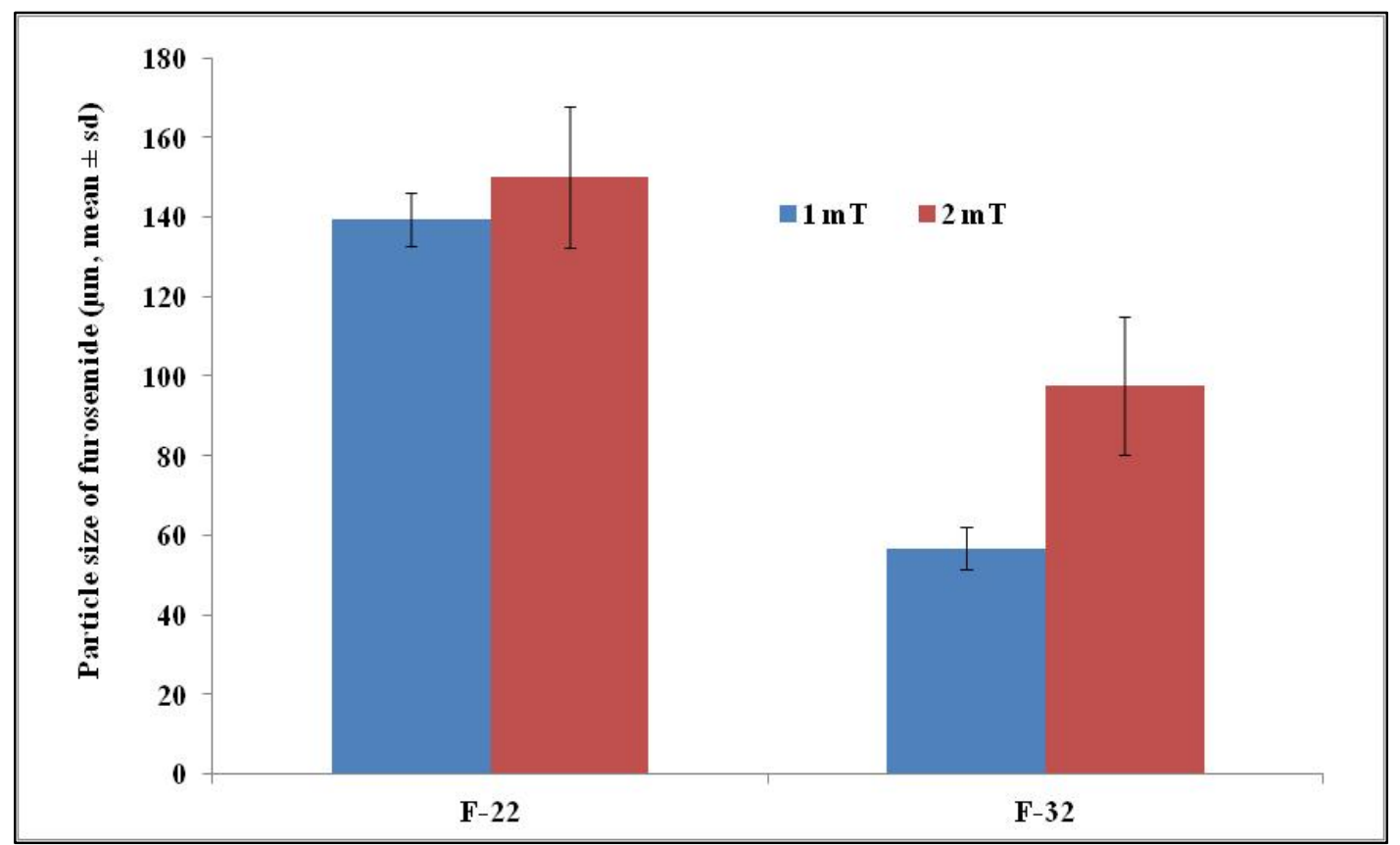

Figure 3-20. Effect of treatment with hexane on furosemide particles during compression 


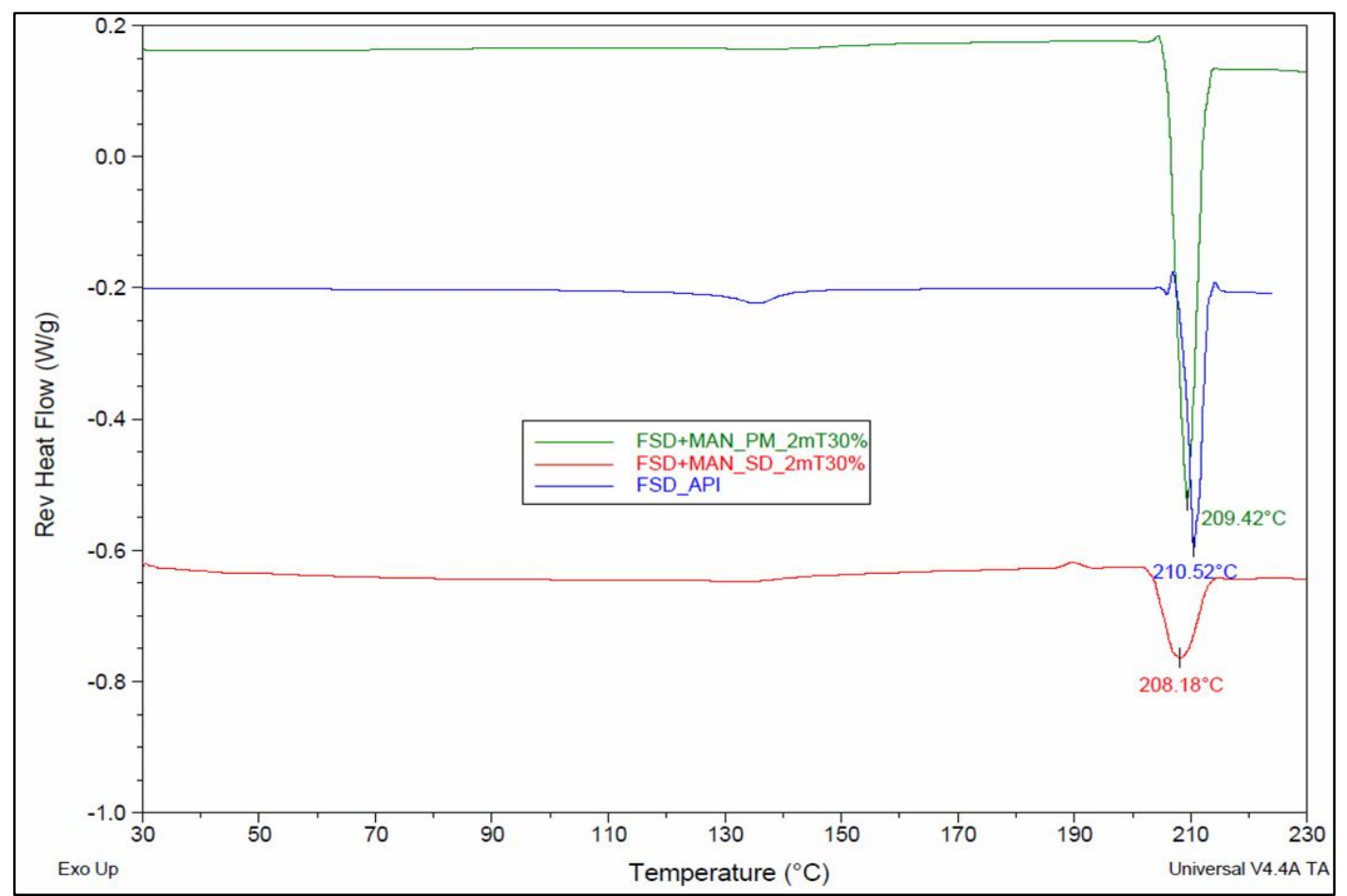

Figure 3-21. MDSC thermograms of furosemide particles after treatment with mannitol spray dried 


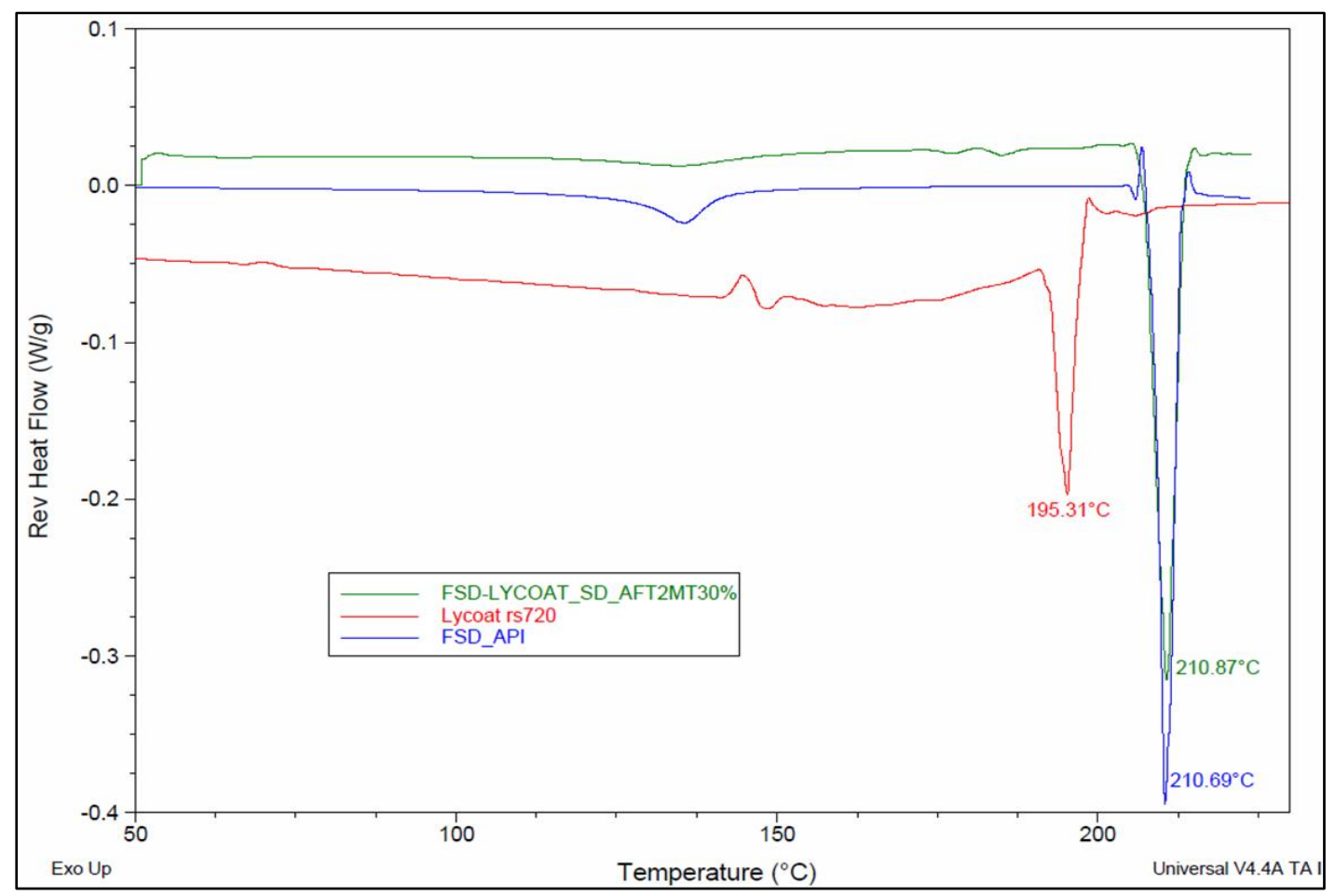

Figure 3-22. MDSC thermograms of furosemide after particles after treatment with Lycoat RS 720 


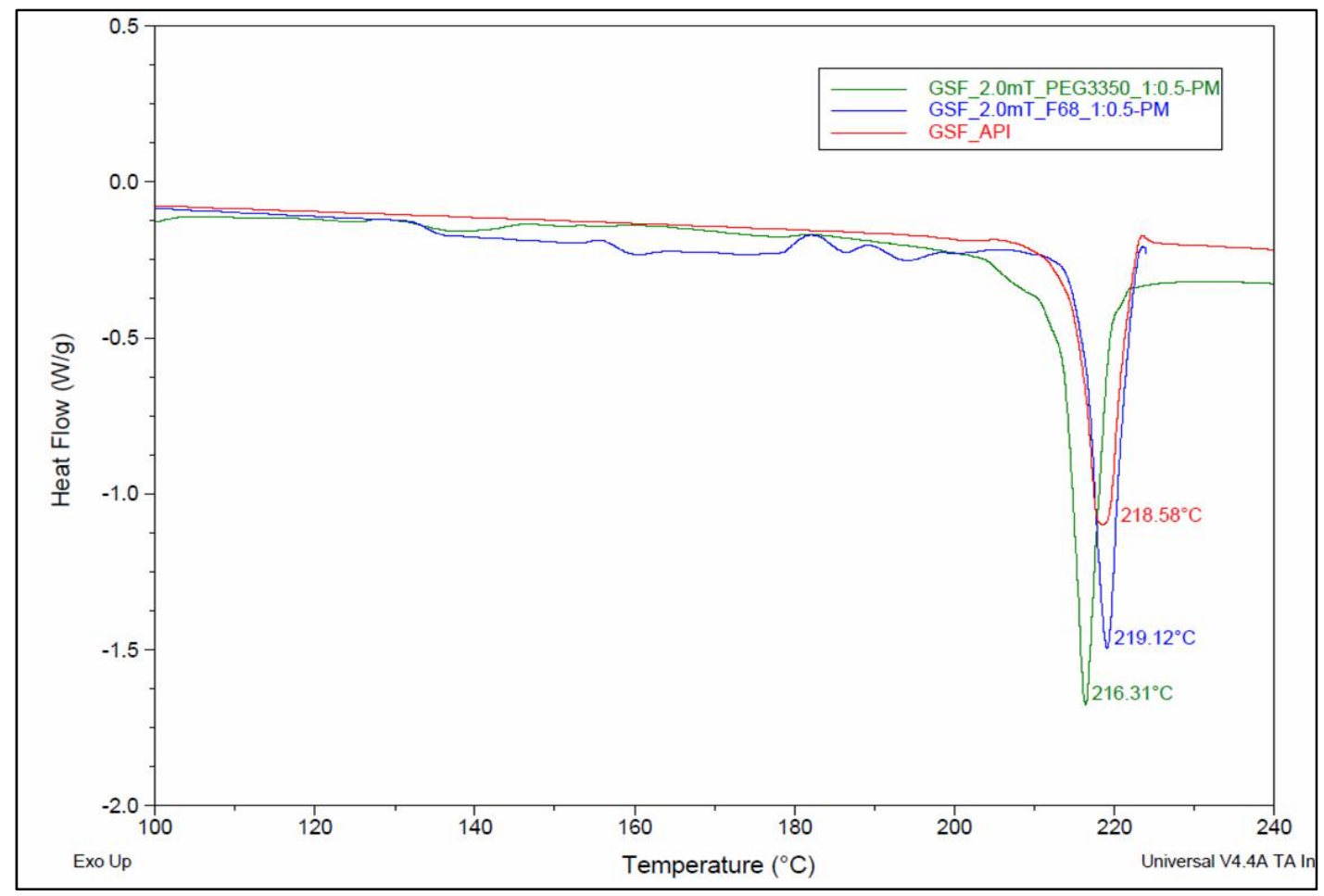

Figure 3-23. MDSC thermograms of griseofulvin after physical treatment with PEG3350 and Lutrol F68 


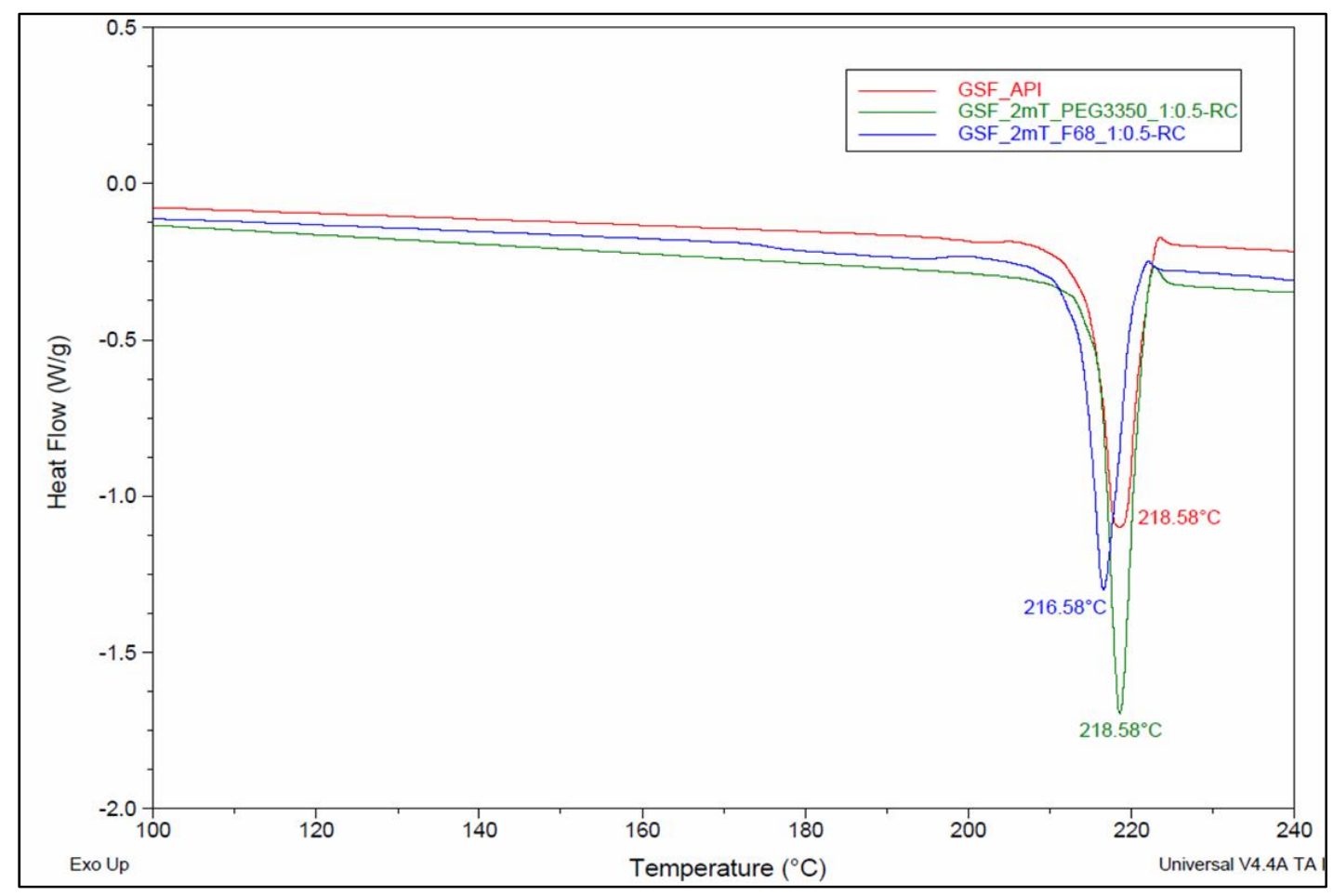

Figure 3-24. MDSC thermograms of griseofulvin particles after roller compaction with PEG 3350 and Lutrol F68 


\subsection{Conclusions}

In conclusion, furosemide and griseofulvin particles agglomeration during compression was prevented by treatment with particle agglomeration inhibitors like PEG 3350 and Lutrol F68 micro. Treating the furosemide particles prior to compression with Lutrol F68 micro by roller compaction process significantly $(p<0.05)$ prevented more agglomeration when compared to physical treatment with PAI's. Modulated differential scanning calorimetry studies provided the evidence of crystallinity and also effect of PAI's on polymorphic transformation during treatment and compression. Hexane treatment for furosemide provided significant $(\mathrm{p}<0.05)$ prevention in agglomeration during compression compared to without any treatment. Increasing level of super disintegrant in preventing the agglomeration during compression study for furosemide showed significant $(\mathrm{p}<0.05)$ prevention when compared at $0.1 \%$ to $1 \%$ and $2 \%$ but not at $1 \%$ to $2 \%(p>0.05)$ which shows its limitation. Spray drying with either mannitol or hydroxyl propyl starch for micronized furosemide significantly $(\mathrm{p}<0.05)$ reduced the agglomeration of API particles during compression. Similarly for griseofulvin physical treatment with Lutrol F68 micro prevented the agglomeration significantly $(p<0.05)$ during compression more successfully whereas with PEG 3350 treatment the prevention was not significant ( $\mathrm{p}>0.05$ ) at $1 \mathrm{mT}$ compression force compared to without any treatment. Roller compaction with Lutrol F68 micro significantly $(\mathrm{p}<0.05)$ prevented the agglomeration of griseofulvin during compression compared to without any treatment whereas with PEG 3350 the prevention was not significant $(p>0.05)$ at $1 \mathrm{mT}$ compression force. This increase in particle size of griseofulvin might be due to formation of griseofulvin-PEG 3350 solid dispersions. Thermal studies revealed that treatment with PAI's and roller compaction processing with PAI's affect the griseofulvin crystalline nature. This polymorphic change might be due to formation of solid dispersion by either treatment with PAI's or during the compression.

In summary, agglomeration of micronized hydrophobic drugs during compression can be prevented by treating with particle agglomeration inhibitors prior to compression. 


\section{LIST OF REFERENCES}

1. Chasseaud LF, Taylor T 1974. Bioavailability of Drugs From Formulations After Oral Administration. Annual Review of Pharmacology 14(1):35-46.

2. Lipinski CA 2002. Poor Aqueous Solubility - an Industry Wide Problem in Drug Discovery. American Pharmaceutical Review 5(3):82-85.

3. Lipinski CA 2000. Drug-like properties and the causes of poor solubility and poor permeability. Journal of pharmacological and toxicological methods 44(1):235249.

4. Lipinski CA, Lombardo F, Dominy BW, Feeney PJ 2001. Experimental and computational approaches to estimate solubility and permeability in drug discovery and development settings. Advanced drug delivery reviews 46(1-3):326.

5. Gullapalli RP 2010. Soft gelatin capsules (softgels). Journal of pharmaceutical sciences 99(10):4107-4148.

6. Wong SM, Kellaway IW, Murdan S 2006. Enhancement of the dissolution rate and oral absorption of a poorly water soluble drug by formation of surfactantcontaining microparticles. International Journal of Pharmaceutics 317(1):61-68.

7. Amidon GL, Lennernas H, Shah VP, Crison JR 1995. A theoretical basis for a biopharmaceutic drug classification: the correlation of in vitro drug product dissolution and in vivo bioavailability. Pharmaceutical research 12(3):413-420.

8. Yu LX, Amidon GL, Polli JE, Zhao H, Mehta MU, Conner DP, Shah VP, Lesko LJ, Chen ML, Lee VH, Hussain AS 2002. Biopharmaceutics classification system: the scientific basis for biowaiver extensions. Pharmaceutical research 19(7):921-925.

9. Noyes AA, Whitney WR 1897. The rate of solution of solid substances in their own solutions. Journal of the American Chemical Society 19(12):930-934.

10. Gibaldi M. 1970. Biopharmaceutics. In Leon Lachman, Herbert A. Lieberman, Joseph L. Kanig, editor The Theory and practice of Industrial Pharmacy, ed., Philadelphia: Lea and Febiger. p 246-250.

11. Dressman JB, Reppas C 2000. In vitro-in vivo correlations for lipophilic, poorly water-soluble drugs. European journal of pharmaceutical sciences : official journal of the European Federation for Pharmaceutical Sciences 11 Suppl 2:S7380. 
12. Godec A, Gaberscek M, Jamnik J 2009. Comment on the Article "A New Understanding of the Relationship between Solubility and Particle Size" by W. Wu and GH Nancollas. J Solution Chem 38(1):135-146.

13. Nancollas GH, Wu WJ 2009. Response to "Comment on "A New Understanding of the Relationship between Solubility and Particle Size" by Godec, A., Jamnik, J., and Gaderscek, M. J Solution Chem 38(1):147-148.

14. Wu WJ, Nancollas GH 1998. A new understanding of the relationship between solubility and particle size. J Solution Chem 27(6):521-531.

15. Leuner C, Dressman J 2000. Improving drug solubility for oral delivery using solid dispersions. Eur J Pharm Biopharm 50(1):47-60.

16. Elamin AA, Ahlneck C, Alderborn G, Nyström C 1994. Increased metastable solubility of milled griseofulvin, depending on the formation of a disordered surface structure. International Journal of Pharmaceutics 111(2):159-170.

17. Rasenack N, Muller BW 2002. Dissolution rate enhancement by in situ micronization of poorly water-soluble drugs. Pharmaceutical research 19(12):1894-1900.

18. Jounela AJ, Pentikainen PJ, Sothmann A 1975. Effect of particle size on the bioavailability of digoxin. European journal of clinical pharmacology 8(5):365370 .

19. Liversidge GG, Cundy KC 1995. Particle size reduction for improvement of oral bioavailability of hydrophobic drugs: I. Absolute oral bioavailability of nanocrystalline danazol in beagle dogs. International Journal of Pharmaceutics 125(1):91-97.

20. Vogt M, Kunath K, Dressman JB 2008. Dissolution enhancement of fenofibrate by micronization, cogrinding and spray-drying: comparison with commercial preparations. Eur J Pharm Biopharm 68(2):283-288.

21. Joshi HN, Tejwani RW, Davidovich M, Sahasrabudhe VP, Jemal M, Bathala MS, Varia SA, Serajuddin AT 2004. Bioavailability enhancement of a poorly watersoluble drug by solid dispersion in polyethylene glycol-polysorbate 80 mixture. Int J Pharm 269(1):251-258.

22. Dannenfelser RM, He H, Joshi Y, Bateman S, Serajuddin AT 2004. Development of clinical dosage forms for a poorly water soluble drug I: Application of polyethylene glycol-polysorbate 80 solid dispersion carrier system. Journal of pharmaceutical sciences 93(5):1165-1175.

23. Sathigari S, Chadha G, Lee YH, Wright N, Parsons DL, Rangari VK, Fasina O, Babu RJ 2009. Physicochemical characterization of efavirenz-cyclodextrin inclusion complexes. AAPS PharmSciTech 10(1):81-87. 
24. Badr-Eldin SM, Elkheshen SA, Ghorab MM 2008. Inclusion complexes of tadalafil with natural and chemically modified beta-cyclodextrins. I: preparation and in-vitro evaluation. Eur J Pharm Biopharm 70(3):819-827.

25. Han HK, Choi HK 2007. Improved absorption of meloxicam via salt formation with ethanolamines. Eur J Pharm Biopharm 65(1):99-103.

26. Farinha A, Bica A, Tavares P 2000. Improved bioavailability of a micronized megestrol acetate tablet formulation in humans. Drug Development and Industrial Pharmacy 26(5):567-570.

27. Atkinson RM, Bedford C, Child KJ, Tomich EG 1962. Effect of particle size on blood griseofulvin-levels in man. Nature 193:588-589.

28. Chokshi RJ, Zia H, Sandhu HK, Shah NH, Malick WA 2007. Improving the dissolution rate of poorly water soluble drug by solid dispersion and solid solution: pros and cons. Drug delivery 14(1):33-45.

29. Loftsson T, Jarho P, Masson M, Jarvinen T 2005. Cyclodextrins in drug delivery. Expert opinion on drug delivery 2(2):335-351.

30. S Stavchansky JM. 1990. Bioavailibility in tablet technology. In H Lieberman LL, JB Scharwtz, editor Pharmaceutical Dosage Forms: Tablets, ed., New York: Marcel Dekker. p 453.

31. Yasuji T, Takeuchi H, Kawashima Y 2008. Particle design of poorly watersoluble drug substances using supercritical fluid technologies. Advanced drug delivery reviews 60(3):388-398.

32. J.A Hersey 1975. Ordered mixing: A new concept in powder mixing practice. Powder Technology 11(1):41-44.

33. Fan LT, Chen Yi-ming, Lai FS 1990. Recent developments in solids mixing. Powder Technology 61(3):255-287.

34. Venables HJ, Wells JI 2001. Powder mixing. Drug Dev Ind Pharm 27(7):599612.

35. Nikolakakis I, Newton JM 1989. Solid state adsorption of antibiotics onto sorbitol. The Journal of pharmacy and pharmacology 41(3):145-148.

36. Nystrom C, Westerberg M 1986. The use of ordered mixtures for improving the dissolution rate of low solubility compounds. The Journal of pharmacy and pharmacology 38(3):161-165. 
37. Nilsson P, Westerberg M, Nyström C 1988. Physicochemical aspects of drug release. V. The importance of surface coverage and compaction on drug dissolution from ordered mixtures. International Journal of Pharmaceutics $45(1-$ 2):111-121.

38. Westerberg M, Jonsson B, Nyström C 1986. Physicochemical aspects of drug release. IV. The effect of carrier particle properties on the dissolution rate from ordered mixtures. International Journal of Pharmaceutics 28(1):23-31.

39. Alderborn G, Nystrom C 1982. Studies on direct compression of tablets. IV. The effect of particle size on the mechanical strength of tablets. Acta pharmaceutica Suecica 19(5):381-390.

40. Vromans H, de Boer AH, Bolhuis GK, Lerk CF, Kussendrager KD 1985. Studies on tabletting properties of lactose, Part I. The effect of initial particle size on binding properties and dehydration characteristics of lactose. Acta pharmaceutica Suecica 22(3):163-172.

41. McKenna A, McCafferty DF 1982. Effect on particle size on the compaction mechanism and tensile strength of tablets. The Journal of pharmacy and pharmacology 34(6):347-351.

42. Jivraj II, Martini LG, Thomson CM 2000. An overview of the different excipients useful for the direct compression of tablets. Pharmaceutical science \& technology today 3(2):58-63.

43. H Leuenberger 1982. The compressibility and compactibility of powder systems. International Journal of Pharmaceutics 12(1):41-55.

44. Marshall K. 1986. Compression and consolidation of powdered solids. In Leon Lachman HAL, Joseph L. Kanig, editor The Theory and practice of Industrial Pharmacy, 3 ed., Philadelphia: Lea and Febiger. p 66-99.

45. Alderborn G, Pasanen K, Nyström C 1985. Studies on direct compression of tablets. XL Characterization of particle fragmentation during compaction by permeametry measurements of tablets. International Journal of Pharmaceutics 23(1):79-86.

46. Duberg M, Nyström C 1986. Studies on direct compression of tablets XVII. Porosity - pressure curves for the characterization of volume reduction mechanisms in powder compression. Powder Technology 46(1):67-75.

47. Nyström C, Alderborn G, Duberg M, Karehill P-G 1993. Bonding Surface area and Bonding Mechanism-Two Important Factors for the Understanding of Powder Comparability. Drug Development and Industrial Pharmacy 19(1718):2143-2196. 
48. Coffin-Beach DP, Gary Hollenbeck R 1983. Determination of the energy of tablet formation during compression of selected pharmaceutical powders. International Journal of Pharmaceutics 17(2-3):313-324.

49. Luangtana-Anan M, Fell JT 1990. Bonding mechanisms in tabletting. International Journal of Pharmaceutics 60(3):197-202.

50. Rumpf H. 1962. The strength of granules and agglomerates. editor Knepper WA, In Agglomeration, ed., New York: John Wiley \& Sons. p 379-418.

51. C Fuhrer 1977. Substance behavior in direct compression. Labo Pharma Probl Techn 25:759-762.

52. Rankell AS, Highuchi T 1968. Physics of tablet compression. XV. Thermodynamic and kinetic aspects of adhesion under pressure. Journal of pharmaceutical sciences 57(4):574-577.

53. Matsumoto T, Kaneniwa N, Higuchi S, Otsuka M 1991. Effects of temperature and pressure during compression on polymorphic transformation and crushing strength of chlorpropamide tablets. The Journal of pharmacy and pharmacology 43(2):74-78.

54. Israelachvili JN. 2011. Intermolecular and Surface Forces. 3 ed., London: Academic Press.

55. De Boer AH, Bolhuis GK, Lerk CF 1978. Bonding characteristics by scanning electron microscopy of powders mixed with magnesium stearate. Powder Technology 20(1):75-82.

56. Bhatia RP, Lordi NG 1979. Electrical conductance of directly compressible materials under pressure. Journal of pharmaceutical sciences 68(2):222-226.

57. Luangtana-anan M, Fell JT 1988. Surface energetics of powders before and after compaction. International Journal of Pharmaceutics 41(3):237-240.

58. Luangtana-Anan M, Fell JT 1987. Surface free energy determinations on powders. Powder Technology 52(3):215-218.

59. Karehill PG, Nyström C 1990. Studies on direct compression of tablets XXI. Investigation of bonding mechanisms of some directly compressed materials by strength characterization in media with different dielectric constants (relative permittivity). International Journal of Pharmaceutics 61(3):251-260.

60. Omelczuk MO, Wang C-C, Pope DG 1997. Influence of micronization on the compaction properties of an investigational drug using tableting index analysis. European Journal of Pharmaceutics and Biopharmaceutics 43(1):95-100. 
61. Hartley PA, Parfitt GD, Pollack LB 1985. The role of the van der Waals force in the agglomeration of powders containing submicron particles. Powder Technology 42(1):35-46.

62. Finholt P, Solvang S 1968. Dissolution kinetics of drugs in human gastric juice-the role of surface tension. Journal of pharmaceutical sciences 57(8):1322-1326.

63. Ampolsuk C, Mauro JV, Nyhuis AA, Shah N, Jarowski CI 1974. Influence of dispersion method on dissolution rate of digoxin-lactose and hydrocortisonelactose triturations. I. Journal of pharmaceutical sciences 63(1):117-118.

64. Aguiar AJ, Zelmer JE, Kinkel AW 1967. Deaggregation behavior of a relatively insoluble substituted benzoic acid and its sodium salt. Journal of pharmaceutical sciences 56(10):1243-1252.

65. Pratibha S. Pilgaonkar MTR, Anilkumar S. Gandhi, Paras R. Jain. 2009. Pharmaceutical Compositions. In USPTO, editor, ed., USA: Rubicon Research Private Limited.

66. Bolhuis GK, Smallenbroek AJ, Lerk CF 1981. Interaction of tablet disintegrants and magnesium stearate during mixing I: Effect on tablet disintegration. Journal of pharmaceutical sciences 70(12):1328-1330.

67. Vorwerg W, Dijksterhuis J, Borghuis J, Radosta S, Kroger A 2004. Film properties of hydroxypropyl starch. Starch-Starke 56(7):297-306.

68. Obara S, Maruyama N, Nishiyama Y, Kokubo H 1999. Dry coating: an innovative enteric coating method using a cellulose derivative. Eur J Pharm Biopharm 47(1):51-59.

69. 1997. Training papers spray drying. ed., Switzarland: BÜCHI Labortechnik AG. Vol B, p 1-19.

70. Fichtner F, Mahlin D, Welch K, Gaisford S, Alderborn G 2008. Effect of surface energy on powder compactibility. Pharmaceutical research 25(12):2750-2759.

71. Gohel MC, Jogani PD 2005. A review of co-processed directly compressible excipients. J Pharm Pharm Sci 8(1):76-93.

72. Latosinska JN, Latosinska M, Medycki W, Osuchowicz J 2006. Molecular dynamics of solid furosemide (4-chloro-2-furfurylamino-5-sulfamoyl-benzoic acid) studied by NMR and DFT methods. Chem Phys Lett 430(1-3):127-132.

73. Pudipeddi M, Serajuddin ATM 2005. Trends in solubility of polymorphs. Journal of pharmaceutical sciences 94(5):929-939. 
74. Beyers H, Malan SF, van der Watt JG, de Villiers MM 2000. Structure-solubility relationship and thermal decomposition of furosemide. Drug Development and Industrial Pharmacy 26(10):1077-1083.

75. Matsuda Y, Tatsumi E 1990. Physicochemical Characterization of Furosemide Modifications. International Journal of Pharmaceutics 60(1):11-26.

76. Matsuda Yoshihisa TE 1989. Physicochemical Characterization of Furosemide Polymorphs and Their Evaluation of Stability against Some Environmental Factors. Journal of pharmacobio-dynamics 12(2):s-38.

77. Shin SC, Kim J 2003. Physicochemical characterization of solid dispersion of furosemide with TPGS. Int J Pharm 251(1-2):79-84.

78. Berthod A, Carda-Broch S, Garcia-Alvarez-Coque MC 1999. Hydrophobicity of Ionizable Compounds. A Theoretical Study and Measurements of Diuretic Octanol-Water Partition Coefficients by Countercurrent Chromatography. Analytical Chemistry 71(4):879-888.

79. 1989. Budavari S, The Merck Index-Encyclopedia of Chemicals, Drugs and Biologicals, ed., Rahway, NJ: Merck and Co., Inc. p 715.

80. Arida AI, Al-Tabakha MM, Hamoury HA 2007. Improving the high variable bioavailability of griseofulvin by SEDDS. Chemical \& pharmaceutical bulletin 55(12):1713-1719.

81. Lin C, Lim J, DiGiore C, Gural R, Symchowicz S 1982. Comparative bioavailability of a microsize and ultramicrosize griseofulvin formulation in man. The Journal of international medical research 10(4):274-277.

82. Straughn AB, Meyer MC, Raghow G, Rotenberg K 1980. Bioavailability of microsize and ultramicrosize griseofulvin products in man. Journal of pharmacokinetics and biopharmaceutics 8(4):347-362.

83. Needham Jr TE 1972. Biopharmaceutics and relevant pharmacokinetics. By John G. Wagner. Drug intelligence publications, Hamilton, IL 62341, 1971. 375 pp. $18.5 \times 26 \mathrm{~cm}$. Price $\$ 15.00$. Journal of pharmaceutical sciences $61(5): 819-819$.

84. Le VN, Leterme P, Gayot A, Flament MP 2006. Influence of granulation and compaction on the particle size of ibuprofen--development of a size analysis method. Int J Pharm 321(1-2):72-77.

85. Kitamori N, Makino T 1979. Evaluation of changes in drug particle size during tableting by measurement of dissolution of disintegrated tablets. The Journal of pharmacy and pharmacology 31(8):501-504. 
86. Carless JE, Sheak A 1976. Changes in the particle size distribution during tableting of sulphathiazole powder. Journal of Pharmacy and Pharmacology 28(1):17-22.

87. Armstrong NA, Griffiths RV 1970. Surface area measurements in compressed powder systems. Pharmaceutica acta Helvetiae 45(9):583-588.

88. Newman AW, Byrn SR 2003. Solid-state analysis of the active pharmaceutical ingredient in drug products. Drug Discovery Today 8(19):898-905.

89. Chan HK, Doelker E 1985. Polymorphic Transformation of Some Drugs Under Compression. Drug Development and Industrial Pharmacy 11(2-3):315-332.

90. Morris KR, Griesser UJ, Eckhardt CJ, Stowell JG 2001. Theoretical approaches to physical transformations of active pharmaceutical ingredients during manufacturing processes. Advanced drug delivery reviews 48(1):91-114.

91. Koivisto M, Heinanen P, Tanninen VP, Lehto VP 2006. Depth profiling of compression-induced disorders and polymorphic transition on tablet surfaces with grazing incidence X-ray diffraction. Pharmaceutical research 23(4):813-820.

92. Khan KA, Rhodes CT 1975. Effect of compaction on particle size. Journal of pharmaceutical sciences 64(3):444-447.

93. Smith HL, Baker CA, Wood JH 1971. Interpretation of dissolution rate maxima: dependence upon tablet compression force. The Journal of pharmacy and pharmacology 23(7):536-538.

94. Hirschorn JO, Kornblum SS 1971. Dissolution of poorly water-soluble drugs II: Excipient dilution and force of compression effects on tablets of a quinazolinone compound. Journal of pharmaceutical sciences 60(3):445-448.

95. Tuladhar MD, Carless JE, Summers MP 1983. The effects of polymorphism, particle size and compression pressure on the dissolution rate of phenylbutazone tablets. Journal of Pharmacy and Pharmacology 35(5):269-274.

96. Sallam E, Ibrahim H, Takieddin M, Baghal T, Saket M, Awad R, Arafat T 1991. Dissolution Characteristics of Interactive Powder Mixtures .4. Effects of Additives on the Dissolution of Griseofulvin from Emcompress Carrier. International Journal of Pharmaceutics 67(3):247-257.

97. Sallam E, Ibrahim H, Takieddin M, Shamat MA, Baghal T 1988. Dissolution Characteristics of Interactive Powder Mixtures. Part Two: Effect of Surface Characteristics of Excipients. Drug Development and Industrial Pharmacy 14(9):1277-1302. 
98. Olsson H, Adolfsson A, Nystrom C 1996. Compaction and measurement of tablets in liquids with different dielectric constants for determination of bonding mechanisms--evaluation of the concept. International Journal of Pharmaceutics 143(Nov 8):233-245.

99. Olsson H, Nystrom C 2001. Assessing tablet bond types from structural features that affect tablet tensile strength. Pharmaceutical research 18(2):203-210.

100. de Villiers MM, van der Watt JG, Lötter AP, Liebenberg W, Dekker TG 1995. Correlation Between Physico-Chemical Properties and Cohesive Behavior of Furosemide Crystal Modifications. Drug Development and Industrial Pharmacy 21(17):1975-1988.

101. Kitamori N, Makino T 1979. Effect of drug content and drug particle size on the change in particle size during tablet compression. The Journal of pharmacy and pharmacology 31(8):505-507.

102. Hancock BC, Parks M 2000. What is the True Solubility Advantage for Amorphous Pharmaceuticals? Pharmaceutical research 17(4):397-404.

103. Hancock BC, Zografi G 1997. Characteristics and significance of the amorphous state in pharmaceutical systems. Journal of pharmaceutical sciences 86(1):1-12.

104. Burt HM, Mitchell AG 1981. Crystal defects and dissolution. International Journal of Pharmaceutics 9(2):137-152.

105. Eriksson M, Alderborn G 1995. The effect of particle fragmentation and deformation on the interparticulate bond formation process during powder compaction. Pharmaceutical research 12(7):1031-1039.

106. Ozeki T, Beppu S, Mizoe T, Takashima Y, Yuasa H, Okada H 2005. Preparation of two-drug composite microparticles to improve the dissolution of insoluble drug in water for use with a 4-fluid nozzle spray drier. Journal of controlled release : official journal of the Controlled Release Society 107(3):387-394.

107. Harnby N 2000. An engineering view of pharmaceutical powder mixing. Pharmaceutical science \& technology today 3(9):303-309.

108. Chiou WL, Riegelman S 1969. Preparation and dissolution characteristics of several fast-release solid dispersions of griseofulvin. Journal of pharmaceutical sciences 58(12):1505-1510.

109. Gonnissen Y, Verhoeven E, Peeters E, Remon JP, Vervaet C 2008. Coprocessing via spray drying as a formulation platform to improve the compactability of various drugs. Eur J Pharm Biopharm 69(1):320-334.

110. Sollohub K, Cal K 2010. Spray drying technique: II. Current applications in pharmaceutical technology. Journal of pharmaceutical sciences 99(2):587-597. 
111. Makai Z, Bajdik J, Erős I, Pintye-Hódi K 2008. Evaluation of the effects of lactose on the surface properties of alginate coated trandolapril particles prepared by a spray-drying method. Carbohydrate Polymers 74(3):712-716.

112. Bolhuis GK, Zuurman K, te Wierik GHP 1997. Improvement of dissolution of poorly soluble drugs by solid deposition on a super disintegrant. II. The choice of super disintegrants and effect of granulation. European Journal of Pharmaceutical Sciences 5(2):63-69.

113. Mitchell SA, Reynolds TD, Dasbach TP 2003. A compaction process to enhance dissolution of poorly water-soluble drugs using hydroxypropyl methylcellulose. International Journal of Pharmaceutics 250(1):3-11.

114. Shah NH, Phuapradit W, Bachynsky M, Infeld MH, Iqbal K, Malick AW 1994. High Energy Ordered Mixture for Improving the Dissolution Rate of Sparingly Soluble Compounds. Drug Development and Industrial Pharmacy 20(5):873-888. 


\section{VITA}

Mr. Suresh Potharaju, son of late Mr. Prasada rao Potharaju and Mrs. Lillimma Potharaju, was born in Andhra Pradesh, India in 1977. He graduated with a Bachelor of Pharmacy degree in 1999 and completed Master of Pharmacy with Pharmacology as major in 2001 from Andhra University, India. Following he joined in Sun Pharmaceutical Industries Ltd in Gujarat, India as a Research Assistant in Pharmacokinetics division and worked for 2 years. Then he worked for 4 years as a Research Scientist in discovery based research company Glenmark Pharmaceuticals Industries Ltd in Mumbai, India. Later in fall 2007 he joined in doctoral program in the department of Pharmaceutical sciences in University of Tennessee Health Science Center. 